\title{
Pratiques du HCR et de ses partenaires en matière de protection communautaire dans les secteurs dans la région de l'Afrique de I'Est, de la Corne de l'Afrique et des Grands Lacs
}

Charles Mballa

Josephine Ngebeh

Machtelt De Vriese

Katie Drew

Abigayil Parr

See next page for additional authors

Follow this and additional works at: https://knowledgecommons.popcouncil.org/departments_sbsr-rh How does access to this work benefit you? Let us know!

\section{Recommended Citation}

Mballa, Charles, Josephine Ngebeh, Machtelt De Vriese, Katie Drew, Abigayil Parr, and Chi-Chi Undie. 2020. "Pratiques du HCR et de ses partenaires en matière de protection communautaire dans les secteurs dans la région de l'Afrique de l'Est, de la Corne de l'Afrique et des Grands Lacs." Nairobi: UNHCR and Population Council. 


\section{Authors}

Charles Mballa, Josephine Ngebeh, Machtelt De Vriese, Katie Drew, Abigayil Parr, and Chi-Chi Undie 


\section{PRATIQUES DU HCR ET DE SES PARTENAIRES EN}

MATIËRE DE PROTECTION

COMMUNAUTAIRE DANS LES

SECTEURS DANS LA RÉGION DE

L'AFRIQUE DE L'EST, DE LA CORNE DE L'AFRIQUE ET DES GRANDS LACS

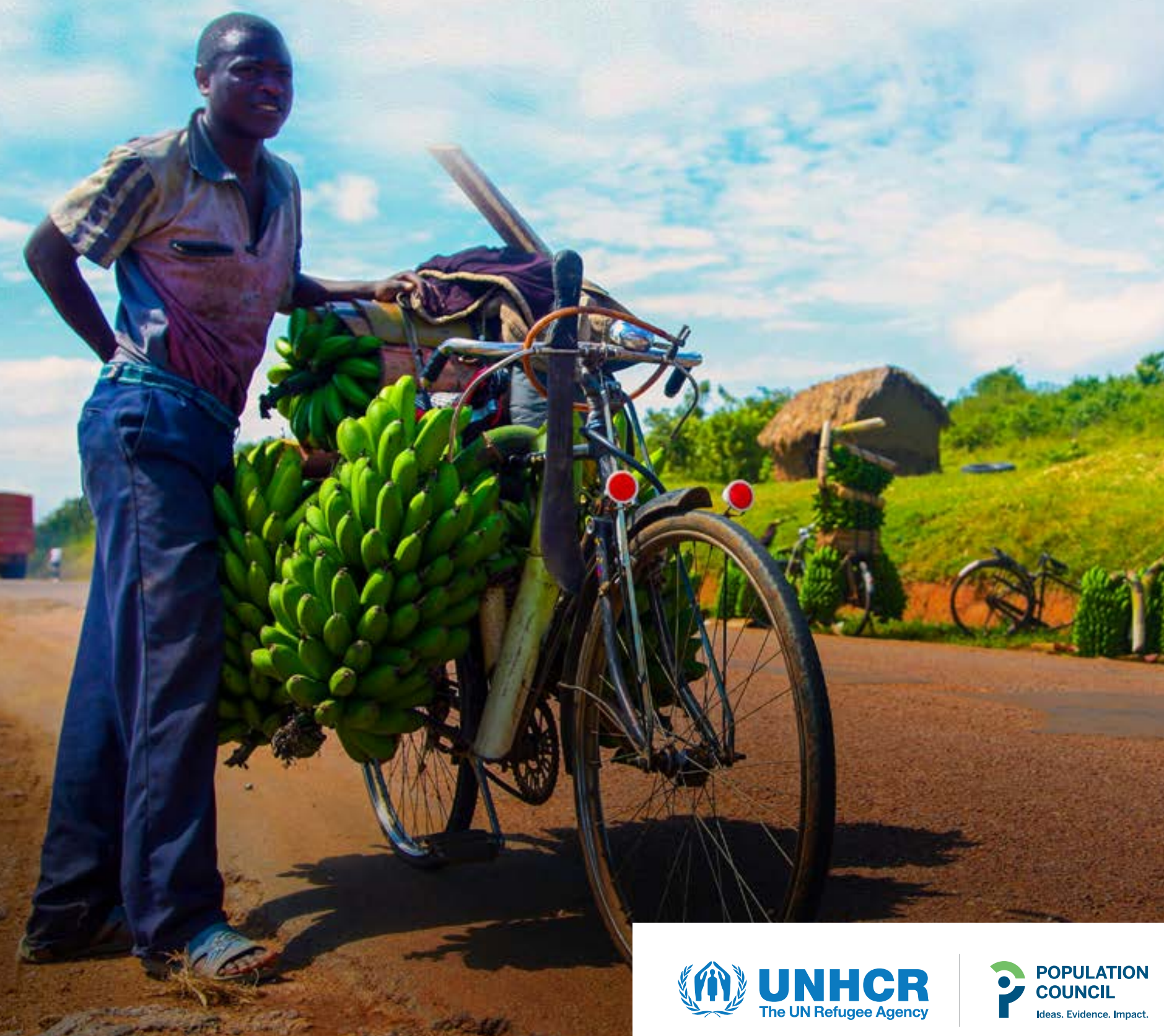




\section{SUGGESTION DE CITATION}

Charles Mballa, Josephine Ngebeh, Machtelt De Vriese, Katie Drew, Abigayil Parr, Chi-Chi Undie. 2020.

Pratiques du HCR et de ses partenaires en matière de protection communautaire dans les secteurs dans la région de l'Afrique de l'Est, de la Corne de l'Afrique et des Grands Lacs HCR et Population Council. 


\section{TABLE DES MATIÈRES}

SIGLES ET ABRÉVIATIONS

AVANT-PROPOS

INTRODUCTION

4

MÉTHODOLOGIE

POINTS SAILLANTS DES PRATIQUES PROMETTEUSES

RECUEIL DES PRATIQUES

PRATIQUES LIÉES À LA COVID

1. PROTECTION DE L'ENFANCE ET DE LA JEUNESSE

9

2. PROTECTION COMMUNAUTAIRE

3. VIOLENCE SEXUELLE ET FONDÉE SUR LE GENRE/ATTÉNUATION DES RISQUES/RÉPONSE

1. PROTECTION DE L'ENFANCE ET DE LA JEUNESSE

2. PROTECTION COMMUNAUTAIRE

3. ÉGALITÉ DES FEMMES ET DES HOMMES

4. VIOLENCE SEXUELLE ET FONDÉE SUR LE GENRE/ATTÉNUATION DES RISQUES/RÉPONSE 


\section{SIGLES ET ABRÉVIATIONS}

AGD Âge, genre et diversité

APE Association des parents d'élèves

ARRA Administration pour les réfugiés et les rapatriés

CNR Conseil norvégien pour les réfugiés

CREC Communication des risques et engagement communautaire

FLM Fédération luthérienne mondiale

MEARL Suivi, évaluation, responsabilisation, recherche et apprentissage

MINEMA Ministère en charge de la gestion des urgences

SCI Save the Children International

SMSPS Santé mentale et soutien psychosocial

UNFPA Fonds des Nations Unies pour la population

VPI Violence physique exercée par le partenaire intime

WASH Eau, hygiène et assainissement 


\section{AVANT-PROPOS}

C

e recueil a été élaboré conjointement par le Bureau régional du HCR pour l'Afrique de l'Est et la Corne de l'Afrique et les Grands Lacs, et Population Council du Kenya. II rassemble un large éventail d'initiatives de protection communautaires innovantes. Le compendium est alimenté par une communauté de pratiques de protection examinée auprès de partenaires, de réfugiés et de communautés d'accueil, ainsi que d'autres parties prenantes. L'Afrique de l'Est et la Corne de l'Afrique ainsi que la région des Grands Lacs accueillent quatre millions de réfugiés. Cette population de réfugiés se caractérise par sa diversité et comprend plus de $80 \%$ de femmes, de filles, de garçons et d'adolescents ayant des besoins de protection multiples.

Dans l'esprit de l'approche globale de la société qui sous-tend le Pacte mondial sur les réfugiés, le recueil est le résultat d'un partenariat de longue date entre le Bureau régional du HCR et Population Council du Kenya, qui a débuté en 2014. Le Bureau régional du HCR et Population Council du Kenya ont collaboré, sur la base d'un cadre de coopération technique qui vise à renforcer la programmation et le partage d'informations dans les situations de réfugiés.

Ce recueil témoigne de la volonté des deux institutions de mettre en œuvre de manière concrète les orientations fondamentales en matière de protection, notamment: la politique et les approches en matière d'âge, de sexe et de diversité (2018) ; les directives sur la responsabilité à l'égard des populations touchées ; les directives du Comité permanent interorganisations, l'inclusion des personnes handicapées dans l'action humanitaire et d'autres cadres de protection qui renforcent les objectifs du Pacte mondial sur les réfugiés réaffirmés par les États Membres de l'Assemblée générale des Nations unies. Le recueil entend également présenter de manière vivante les approches multipartites dans la poursuite de résultats de protection collective, et mettre en lumière la prévention et les réponses à la COVID-19 qui tiennent compte de l'âge, du sexe et de la diversité, de manière à ce qu'elles se renforcent mutuellement.

Nous espérons sincèrement que ce recueil servira d'outil pratique aux parties prenantes pour améliorer la fourniture de protection, promouvoir la participation directe des personnes relevant de la compétence du HCR et des communautés d'accueil à la prise de décision et documenter les pratiques innovantes d'une manière qui ait un impact tangible et significatif sur la vie et les divers besoins de protection de millions de réfugiés dans la région.

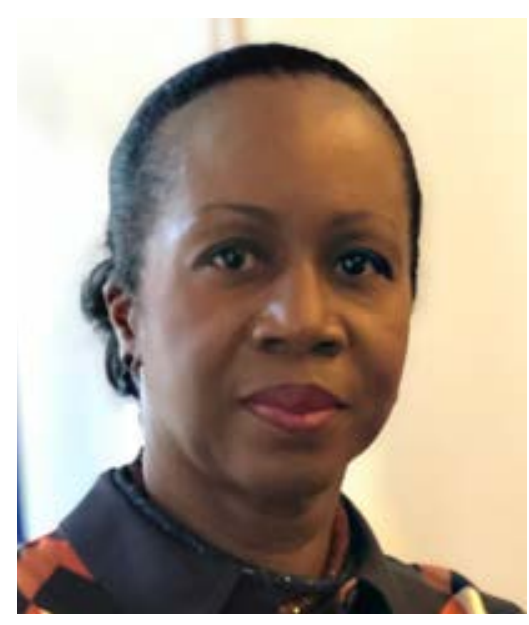

Clementine Awu Nkweta Salami Directeur Bureau régional du HCR pour la région de l'Est, de la Corne de l'Afrique et des Grands Lacs

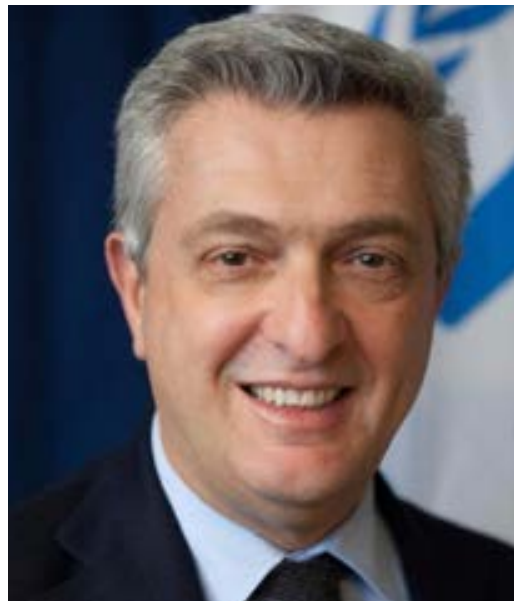

Filippo Grandi

Haut-Commissariat des Nations unies pour les réfugiés HCR

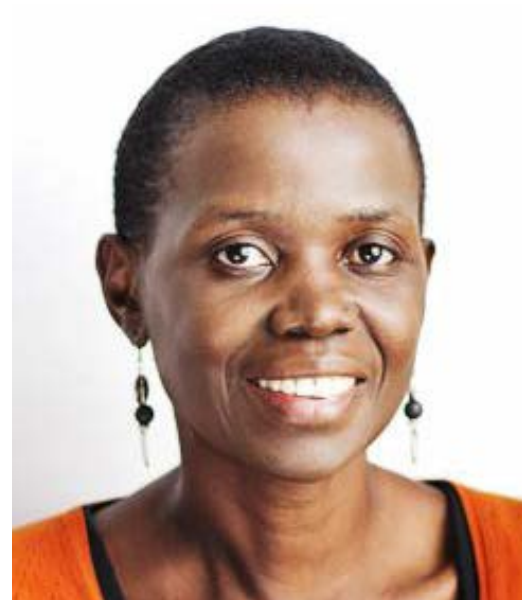

Harriet Birungi

Directeur de pays et associé principal du Population Council du Kenya 


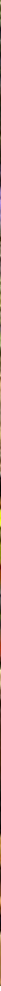

\section{INTRODUCTION}

C

e recueil est une synthèse des informations régionales sur les pratiques prometteuses, les pratiques émergentes et les études de cas qui font partie de la réponse du Haut-Commissariat des Nations Unies pour les réfugiés (HCR) et de ses partenaires aux besoins des réfugiés, des personnes déplacées et des communautés d'accueil dans la région de I'Afrique de l'Est, de la Corne de l'Afrique et des Grands Lacs. Le rapport sur ces pratiques vise plusieurs objectifs. II permet de promouvoir les échanges, l'apprentissage et le renforcement des capacités sud-sud et à l'échelle mondiale, tout en inspirant des idées nouvelles au HCR et à ses partenaires concepteurs et exécutants de programmes pour résoudre des problèmes communs.

La pandémie de la COVID-19 rend ce recueil d'autant plus déterminant qu'à l'heure actuelle, les opérations visent à répondre aux besoins des personnes relevant de la compétence du HCR dans un contexte de confinement sans précédent et de réglementations distanciation physique, qui ont complètement bouleversé les mécanismes de prestation de services dans les situations de réfugiés. Il est important de prendre note des innovations qui ont vu le jour dans ces circonstances et de les partager entre les secteurs et les domaines thématiques. Les interventions appliquées avec succès dans un secteur peuvent être utilisées pour résoudre des problèmes dans un autre ; toutefois, leur recensement permet d'autre part de mieux comprendre les forces et les lacunes des programmes. Des thèmes communs aux opérations nationales sont également mis en lumière.

En plus des pratiques adoptées en réponse à la COVID-19, le recueil consigne également les interventions qui ont précédé la pandémie et qui se poursuivront donc au-delà lors du retour à la normale. Cette approche permet de s'assurer que les pratiques innovantes en cours avant la COVID-19 ne sont pas négligées - en particulier compte tenu de leur importance, étant donné que les restrictions liées à la pandémie évoluent et s'atténuent avec le temps.

Malgré les efforts concertés (décrits dans la section suivante) pour rendre le recueil aussi complet et exhaustif que possible, il est important de noter que ce document n'est en aucun cas représentatif de toutes les pratiques en cours dans les opérations du HCR sur le terrain (car les communications d'informations par le HCR et ses partenaires qui ont permis de réaliser le recueil ont été faites de façon volontaire). II représente plutôt un point de départ important pour avoir un aperçu du large éventail d'efforts qui offrent des solutions émergentes aux nouveaux défis. Le recueil est donc un document vivant qui reconnaît l'évolution des solutions des programmes, et qui, il faut l'espérer, stimulera la production de plus de rapports et de résultats pour témoigner des réalisations, ainsi que de la croissance et de la consolidation des pratiques qui passent souvent inaperçues. Le recueil met en lumière les personnes relevant de la compétence du HCR1 et les communautés d'accueil comme des partenaires de bonne foi du HCR qui conceptualisent ou co-conceptualisent de nombreuses pratiques recensées en fonction de leurs nouveaux besoins. La créativité de ces partenaires, du HCR, des pouvoirs publics et des agences d'exécution du HCR y est soulignée.

1 This includes refugees, asylum seekers, Internally Displaced People (IDP), returnees and statelesss persons. 


\section{MÉTHODOLOGIE}

e Bureau régional du HCR pour l'Afrique de l'Est, la Corne de I'Afrique et les Grands Lacs supervise un total de 11 pays, à savoir : Burundi, Djibouti, Érythrée, Éthiopie, Kenya, Ouganda, Rwanda, Somalie, Soudan, Soudan du Sud et Tanzanie. L'unité de protection communautaire du HCR a invité toutes les opérations nationales à identifier les pratiques notables avant et pendant la COVID-19. Afin de faciliter le processus d'identification, le HCR a mis à la disposition de ses points focaux sectoriels une échelle d'auto-évaluation2 et ces derniers ont été invités à la faire circuler avec le document de méthodologie du HCR pour la collecte des pratiques3 dans leurs opérations. Des équipes polyvalentes ont été recommandées comme mécanisme pour assurer que l'identification des pratiques soit aussi inclusive que possible, et que les personnes relevant de la compétence du HCR soient impliquées dans le processus, aux côtés des partenaires d'exécution. Un modèle de présentation des pratiques4 du HCR a également été recommandé comme format à utiliser par les opérations pour rédiger et présenter leurs pratiques. Afin d'encourager les communications, ces recommandations n'ont pas été présentées comme des prescriptions.

Le document de méthodologie du HCR pour la collecte des pratiques définit trois catégories pour la classification des pratiques sur le terrain ; à savoir, les pratiques prometteuses, les pratiques émergentes et les études de cas. Ces catégories sont définies ci-dessous.

\begin{tabular}{|l|l|}
\hline Catégorie & Définition \\
\hline Pratique prometteuse & $\begin{array}{l}\text { Une pratique dont il est prouvé qu'elle fonctionne bien et produit des résultats durables et qui a } \\
\text { un potentiel de protection et/ou de transformation pour les individus et/ou les communautés, } \\
\text { comme le démontrent des résultats fiables. Elle peut servir de modèle à reproduire et à mettre } \\
\text { à l'échelle. }\end{array}$ \\
\hline Pratique émergente & $\begin{array}{l}\text { Une pratique qui montre les premiers signes de résultats positifs pour transformer positivement } \\
\text { la vie des individus et/ou des communautés. }\end{array}$ \\
\hline Études de cas & $\begin{array}{l}\text { Une pratique, ou une partie de pratique, qui a obéi à des critères définis ou tenté de le } \\
\text { faire (et a rencontré des défis) sans qu'il soit nécessaire de fournir des faits probants ou de } \\
\text { porter un jugement sur ses résultats ou sa durabilité. Elle peut fournir des indications et des } \\
\text { enseignements pour la programmation future, même si elle ne répond pas à certains aspects } \\
\text { des critères. }\end{array}$ \\
\hline
\end{tabular}

Comme indiqué dans le document de méthodologie du HCR,

«Les pratiques prometteuses sont celles qui répondent à des normes plus élevées, qui sont étayées par des faits probants et seront soumises à un examen plus approfondi. Les pratiques émergentes sont celles qui montrent des signes ou un potentiel de résultats. ... Les études de cas sont définies de manière plus vague comme des pratiques qui répondent à des exigences de base et qui montrent des signes ou ont le potentiel de produire des résultats. Elles peuvent se situer à différents stades du cycle de programmation et présenter des résultats plus ou moins probants ou de qualité variable. Les [pratiques émergentes et les études de cas] méritent d'être consignées, car elles peuvent éclairer la programmation future $» 5$.

Deux réunions avec les points focaux sectoriels du HCR ont été organisées pour clarifier l'objectif du recueil, renforcer son importance et augmenter le nombre de communications d'opérations par pays.

2 UNHCR (2019). Methodology and Background Document: Collecting Practices and Case Studies - Child Protection, Community-Based Protection, Gender Equality, Sexual and Gender-Base Violence, pp. 12-18.

3 Ibid.

4 Ibid., pp. 16-17.

5 UNHCR (2019). Methodology and Background Document: Collecting Practices and Case Studies - Child Protection, Community-Based Protection, Gender Equality, Sexual and Gender-Base Violence, p. 7. 
En septembre 2020, un jury de trois personnes6 a évalué indépendamment chaque communication avant de la classer selon sa conformité avec les définitions de « pratique prometteuse », « pratique émergente » et «étude de cas ». Pour les cas où les évaluations des jurés n'étaient pas similaires, l'évaluation donnée par la majorité (2 sur 3) a été utilisée comme catégorisation finale. En cas de conflit d'intérêts (dû à l'affiliation/implication des évaluateurs dans une pratique en cours d'évaluation), les jurés concernés se sont abstenus de participer à l'évaluation en question. En outre, trois ateliers régionaux virtuels ont été organisés en septembre 2020, réunissant les opérations nationales pour discuter de leurs communications, faire des présentations sur les pratiques sélectionnées, renforcer la compréhension des soumissionnaires des trois catégories de pratiques et parvenir à un consensus sur la catégorisation finale attribuée par les jurés. Le premier atelier régional a porté sur toutes les pratiques soumises par l'Érythrée, Djibouti, la Somalie, le Sud-Soudan et le Soudan, tandis que le deuxième s'est concentré sur l'Éthiopie, le Kenya et l'Ouganda. Le troisième atelier a réuni le Burundi, le Rwanda et la Tanzanie. À la suite des ateliers, les candidats ont eu plusieurs jours pour soumettre des renseignements complémentaires sur une pratique soumise, s'il existait des faits probants qui n'avaient pas été décrites dans le modèle de communication, mais qui pouvaient contribuer à la catégorisation finale de la pratique. Les trois ateliers régionaux virtuels ont rassemblé un total de 120 participants.

Toutes les communications ont été examinées, que le modèle de fournit à cet effet ait été utilisé ou non pour les de pratiques (les seules exceptions étaient celles qui tentaient de témoigner des pratiques en une ou deux phrases, et qui ne fournissaient donc pas suffisamment de renseignements). Bien que cela ait représenté un défi pour la présentation uniforme des pratiques, cela a permis d'élargir la participation et d'assurer l'inclusion de pratiques innovantes de toute la région : Le recueil comprend près de 70 pratiques soumises par 10 opérations nationales.

Le recueil est structuré en deux sections principales: Pratiques liées à COVID-19, et pratiques non liées à COVID-19. Chaque section principale est ensuite organisée selon les domaines thématiques du HCR, à savoir : protection de l'enfance et de la jeunesse, protection communautaire, égalité des femmes et des hommes, et violence sexuelle et fondée sur le genre/atténuation des risques/réponse/atténuation des risques/réponse. II convient de noter que la responsabilité envers les personnes touchées touche tous les domaines thématiques abordés dans ce recueil.

Chaque pratique est décrite, avec un aperçu des résultats également inclus pour les pratiques prometteuses, et fréquemment, pour les pratiques émergentes. Par souci de concision, les descriptions des pratiques n'approfondissent pas des questions telles que les difficultés rencontrées lors de la mise en œuvre, mais tentent de fournir suffisamment de renseignements pour que le lecteur comprenne les objectifs et les composantes de chaque intervention. Les coordonnées sont ensuite fournies pour permettre aux lecteurs de contacter directement les personnes qui ont conceptualisé les pratiques et/ou qui les mettent en œuvre de première main. En effet, on espère que de telles interactions auront lieu dans les opérations nationales et entre celles-ci, et que le recueil cultivera continuellement la recherche et le partage d'informations.

Le chevauchement entre les domaines thématiques est remarquable et donne lieu à des catégorisations de pratiques qui ne sont pas nécessairement sans faille dans l'ensemble. Néanmoins, cette réalité démontre l'interdépendance des domaines thématiques, ce qui est une bonne chose.

6 Two Innovation Officers, with the UNHCR EHAGL Bureau, and with the Innovation Service (Global), respectively; and a Population Council Senior Associate. All reviewers were based in Nairobi, Kenya. 


\section{POINTS SAILLANTS DES PRATIQUES PROMETTEUSES}

C ette section introductive du recueil se termine par les points saillants des pratiques prometteuses identifiées dans la région sur la base des communications volontaires des parties prenantes. Elle se concentre sur les résultats ou les impacts générés par ces pratiques. Un compte rendu plus détaillé de chaque pratique soumise pour inclusion dans le recueil (y compris toutes les pratiques prometteuses) est présenté dans la suite du document.

\section{Pratique prometteuse en Éthiopie : Évaluation et programmation de l'éducation et approche d'engagement communautaire}

\section{Résultats}

- Depuis son adoption en 2015, cette approche a permis d'améliorer la fréquentation et la rétention scolaires.

- Le taux de fréquentation du cycle primaire était de 83 \% en 2019 et le nombre d'inscriptions au niveau secondaire dans les écoles de réfugiés et de communautés d'accueil a augmenté de $53 \%$ au cours du premier semestre 2020.

\section{Pratique prometteuse au Rwanda : Groupe de travail sur l'hygiène communautaire et effort conjoint de la communauté, y compris des musiciens locaux, dans la prévention de la COVID-19}

\section{Résultats}

- Dans le cadre des actions conjointes de prévention de la COVID-19, la communauté des réfugiés a construit 122 postes de lavage des mains supplémentaires pour promouvoir l'hygiène dans le camp.

- Les résultats de l'enquête les connaissances, les attitudes et les pratiques (CAP) ont indiqué que les connaissances relatives à la COVID-19 dans le camp étaient généralement élevées, et que la majorité des réfugiés du camp concerné reconnaissent que la communication sanitaire communautaire est une source importante d'information. De plus, les réfugiés ont obtenu un score plus élevé à l'enquête CAP en ce qui concerne le lavage des mains (comparé à d'autres comportements d'hygiène), ce qui témoigne en partie de l'impact positif de l'effort communautaire conjoint pour établir des postes de lavage des mains.

- Les femmes ont obtenu un score inférieur à celui des hommes sur les connaissances liées à la COVID-19, en raison de l'analphabétisme et de l'accès limité au téléphone/à la radio.

\section{Pratique prometteuse au Rwanda : Associations d'épargne et de prêt volontaires}

\section{Résultats}

- Le camp de réfugiés de Mahama compte actuellement 15 associations formées principalement par des personnes handicapées, bien que la participation soit ouverte à tous les membres de la communauté

- Les membres de la communauté, y compris les dirigeants, ont adhéré à des associations génératrices de revenus initiées par des personnes handicapées. L'effectif total des associations est de 361 membres de la communauté (159 hommes, 202 femmes). Parmi eux, 90 sont des personnes handicapées.

- Au total, 15 associations ont ouvert des comptes bancaires.

- Les associations sont maintenant sollicitées par diverses parties prenantes (par exemple, le gouvernement rwandais et les ambassades étrangères) pour participer à des compétitions, des expositions et des cérémonies de remise de prix dans les camps, ainsi qu'à d'autres occasions spéciales. Lors des réunions de masse et des diverses journées internationales, des créneaux horaires sont alloués aux réfugiés handicapés, à leurs familles et à leurs partenaires pour partager des témoignages sur les effets des associations qui ont changé leur vie.

- Les communautés témoignent que les réfugiés handicapés ont également la capacité et la force de soutenir leur famille et la communauté dans son ensemble. 


\section{Pratique prometteuse au Rwanda : Identification des survivants et réponse par le dépistage}

\section{Résultats}

- En 2017, les établissements de santé participants dans deux camps du Rwanda ont connu une augmentation de $300 \%$ de la proportion de survivantes identifiées et ont eu accès à des soins complets liés à la violence sexuelle et fondée sur le genre dans les deux mois suivant le début de l'intervention de dépistage.

- En 2019, dans les cinq camps de réfugiés participants au Rwanda, 326 personnes ont été identifiées comme étant survivantes de la violence sexuelle et fondée sur le genre par dépistage, et 276 ont été orientées vers les services d'intervention compétents en la matière.

- En 2019, les établissements de santé participants dans les camps de réfugiés de quatre pays réunis (dont le Rwanda) ont connu une augmentation de $129 \%$ de la proportion de survivantes identifiées et ont eu accès à des soins complets liés à la violence sexuelle et fondée sur le genre dans les six mois suivant le début du dépistage.

- Dans les cinq camps du Rwanda où le dépistage systématique de la violence sexuelle et fondée sur le genre est effectué, les partenaires en charge de la réponse en la matière signalent une augmentation soudaine des cas reçus, qui serait liée à l'augmentation significative de la détection des survivantes et de l'accès aux soins. Les données qualitatives recueillies auprès des clients et des prestataires du centre de santé indiquent que les femmes et les filles savent maintenant qu'en plus du partenaire/agence de lutte contre la violence sexuelle et fondée sur le genre, elles peuvent également signaler les incidents qui y sont liés au centre de santé. Cela a contribué à augmenter le nombre de cas de violence sexuelle et fondée sur le genre détectés.

- Outre le Rwanda, l'intervention a été reproduite par le HCR et ses partenaires d'exécution au Sud-Soudan, en Ouganda et en Zambie.

- En collaboration avec Population Council, le HCR a organisé une première série de réunions avec les partenaires de l'éducation, le district, les enseignants et les élèves, afin de préparer le terrain pour l'introduction d'une intervention de dépistage des abus sexuels sur les enfants dans les établissements scolaires des camps de Mahama, Mugombwa et Kigeme. Cette initiative, également développée et évaluée initialement au Kenya par le Population Council et le Kenyatta National Hospital, est en attente en raison de la COVID19.

\section{Pratique prometteuse en Ouganda : L'« Alliance de villages de tolérance zéro »}

\section{Résultats}

- Les résultats de l'évaluation de cette intervention communautaire de prévention de la violence sexuelle et fondée sur le genre ont démontré une réduction statistiquement significative des cas de violence physique exercée par le partenaire intime (VPI) (pour les hommes et les femmes), de la VPI sexuelle (pour les hommes), de la violence physique exercée par un non-partenaire (pour les hommes et les femmes) et de la violence sexuelle exercée par un non-partenaire (pour les femmes).

- À ce jour, l'intervention de l'Alliance de villages de tolérance zéro a été mise en œuvre dans une quinzaine de villages de réfugiés en Ouganda. Des préparatifs de base (y compris la formation des partenaires d'exécution, la sensibilisation des responsables communautaires et/ou la création de forums des parties prenantes, etc.) ont également été effectués pour en fin de compte introduire l'intervention dans les milieux de réfugiés à Djibouti, en Éthiopie et en Zambie. 


\section{RECUEIL DES PRATIQUES PRATIQUES LIÉES À LA COVID}

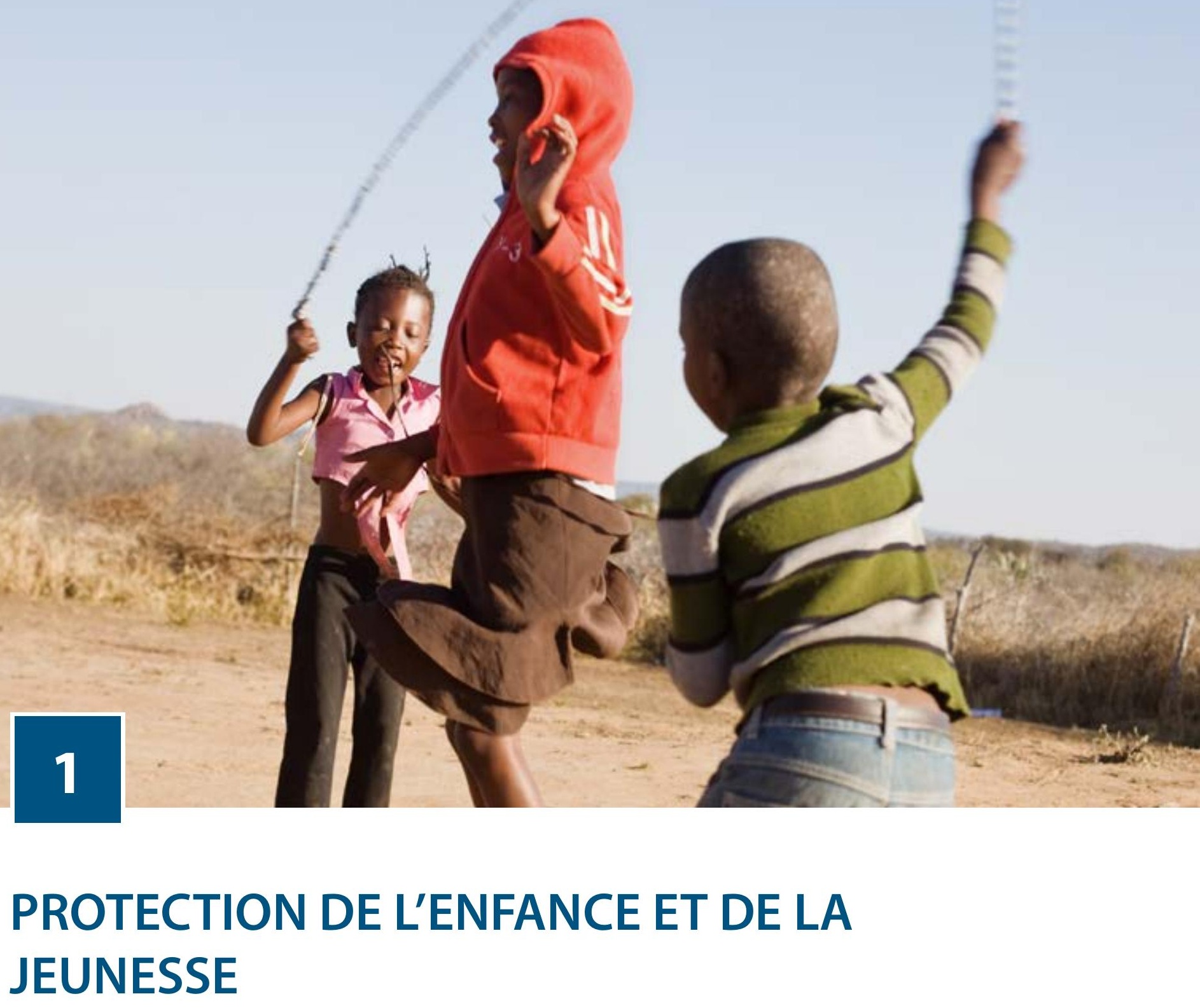

Pratique émergente à Djibouti : Gestion à distance des dossiers de protection de l'enfance en faisant appel à des travailleurs rémunérés au rendement et à des membres de la protection communautaire

a détection des cas COVID-19 dans les camps de réfugiés a conduit à des mesures de confinement imposées par le gouvernement, qui ont interdit l'entrée (et la sortie) de ces lieux aux réfugiés, aux partenaires d'exécution et au HCR. Cela a conduit à une situation sans précédent dans laquelle certains enfants ont été séparés de leurs parents (par exemple, dans les cas où les parents avaient déjà quitté les camps pour le travail ou des soins médicaux).

Cette pratique émergente implique donc que le HCR et son partenaire d'exécution collaborent à distance avec les travailleurs rémunérés au rendement et les membres de la protection communautaire intervenant dans le domaine de 
la protection des enfants dans les camps de réfugiés pour suivre la gestion des cas individuels des enfants séparés, des mineurs non accompagnés, des enfants en danger et des enfants négligés ou abandonnés.

Les composantes spécifiques de cette intervention sont les visites à domicile, la sensibilisation et la collecte de données sur la gestion des cas de protection de l'enfance, comme indiqué ci-dessous :

\section{Visites à domicile}

- Évaluation des besoins et de la situation de vie des enfants dans les camps de réfugiés, y compris le logement, le soutien financier, l'aide alimentaire et l'assistance médicale.

- Identification des enfants ayant des besoins spécifiques et des problèmes de protection, tels que l'expérience de violence (physique, sexuelle), le mariage précoce et la grossesse.

o Identification des enfants négligés, abandonnés, séparés, non accompagnés et orphelins.

\section{Sensibilisation}

- ensibiliser les communautés aux questions de protection qui les préoccupent, telles que les droits de l'enfant, les abus sexuels et physiques, le travail des enfants et le mariage précoce.

- Sensibilisation de la communauté aux mesures de protection contre la COVID-19.

\section{Collecte de données}

- Enregistrement du nombre d'enfants dans le camp qui sont séparés, orphelins, abandonnés ou non accompagnés.

- Rapport hebdomadaire sur les activités menées dans le camp et sur la situation actuelle du camp.

- Identification des cas liés à la protection de l'enfance qui nécessitent une assistance immédiate et un signalement.

- Réalisation d'une enquête auprès des ménages pour évaluer le bien-être psychosocial de leurs membres (y compris les enfants) en rapport à l'impact des restrictions imposées en raison de la COVID-19 sur la vie quotidienne des réfugiés.

\section{Résultats}

Au total, 41 enfants survivants ont été identifiés grâce à cette intervention au cours de la période initiale de 4 mois après le début de la COVID-19. Les survivants sont inscrits au programme de gestion des cas en cours.

Partenaire(s) : International Children's Action Network

Pour de plus amples informations, contacter :

Laura De Somer : desomer@unhcr.org

Arnaud Ntahompagaze : ntahompa@unhcr.org

Helene Chege Wangui : helen.wangui.c@gmail.com

\section{Pratique émergente en Tanzanie : La radio communautaire au service de l'éducation pendant la COVID-19}

$\int d$ ans les camps de réfugiés en Tanzanie, une approche multidimensionnelle est utilisée pour assurer la continuité de la scolarisation pendant la pandémie de la COVID-19. L'approche implique I'utilisation d'un programme d'apprentissage à distance via la radio communautaire, associé à la préparation d'un paquet éducatif à mettre à la disposition des ménages.

Le Groupe de travail sur l'éducation des réfugiés a tenu des consultations avec les pouvoirs publics et obtenu l'autorisation de produire et de mettre en ondes un programme radiodiffusé d'éducation dans le but de répondre aux besoins des réfugiés. Une fois l'autorisation obtenue, le Groupe de travail a commencé à travailler avec Radio Kwizera sur la planification de l'émission. Le processus de planification a nécessité la prise en compte des contraintes financières et technologiques. L'accès aux postes de radio et autres appareils étant limité dans les camps de réfugiés, le Groupe de travail s'efforce d'acquérir et de distribuer des appareils radio fonctionnant à l'énergie solaire dans les foyers. Les partenaires du HCR dans le domaine de l'éducation sont également en train de reprogrammer leurs budgets et leurs approches pour répondre aux nouveaux besoins.

En outre, des kits d'enseignement à domicile, comprenant des photocopies de manuels scolaires basés sur le programme d'études, sont en cours de préparation pour l'apprentissage à domicile, ciblant principalement les élèves du secondaire. 
Partenaire(s) :

Groupe de travail sur l'éducation des réfugiés

Radio Kwizera

Pour de plus amples informations, contacter :

Tamar Joanian : joanian@unhcr.org

\section{Pratique émergente en Ouganda : Enfants ambassadeurs pour la lutte contre la COVID-19}

a pandémie de la COVID-19 a fait apparaître la nécessité de lancer l'éducation communautaire pour assurer

la conformité avec les directives du Ministère de la santé, notamment en ce qui concerne le lavage des mains, le confinement à domicile et distanciation physique. En réponse, World Vision a lancé une initiative de sensibilisation de la communauté en collaboration avec les responsables communautaires, notamment les membres du Comité de protection de l'enfance et les chefs de quartier qui distribuaient du savon aux ménages vulnérables pour leur permettre de se laver les mains. Au cours du processus de distribution aux ménages, il a été observé que les enfants se moquaient des distributeurs domestiques en criant « corona ». Au sein de l'équipe de distribution dans les ménages, «William », un enfant réfugié sud-soudanais, qui se trouvait faire partie d'un club d'hygiène créé par World Vision dans son école, était le seul membre qui suscitait l'intérêt des enfants, et qu'ils semblaient désireux d'écouter. Par conséquent, les enfants sont devenus un élément essentiel du programme. L'ampleur de leur implication dans le programme a également été soulignée par le contexte de confinement pendant la pandémie, qui a perturbé leurs activités habituelles, ce qui a eu pour effet de les rendre oisifs, ne sachant pas où aller, ou incapable jouer et interagir avec leurs amis. Cela a également créé de nouveaux facteurs de stress pour les parents et les personnes qui s'occupent des enfants, qui ont souvent dû soit renoncer à travailler, soit trouver de nouvelles solutions de garde d'enfants.

Cette intervention visait à donner aux enfants les moyens de communiquer efficacement avec leurs pairs sur la nouvelle situation créée par la COVID-19, et de s'engager à respecter les mesures préventives. L'intervention a commencé par William, le membre du club d'hygiène amené à apporter son soutien en tant qu'ambassadeur. Son rôle était de sensibiliser les autres enfants du camp ; pour ce faire, un mégaphone a été mis à sa disposition pour faire passer des messages dans différents villages, sensibilisant ses camarades à la prévention de la COVID-19. Depuis lors, 19 enfants ambassadeurs (9 filles et 10 garçons) ont participé à une formation de deux jours sur la COVID-19, dispensée par deux agents de santé et un membre du personnel d'une organisation partenaire d'exécution qui se concentre sur la communication avec les communautés. La session de formation renforce la capacité des enfants à diffuser des informations relatives à la COVD-19 auprès de leurs pairs et dans leurs communautés. À l'instar de William, les enfants formés reçoivent des mégaphones pour sensibiliser d'autres enfants dans leur quartier. Ils reçoivent également des dépliants contenant des messages adaptés aux enfants, élaborés par le Ministère de la santé et appris par les enfants dans le cadre de la formation.

\section{Résultats}

En juin 2020, 948 enfants (458 filles et 490 garçons) avaient reçu des messages sur la COVID-19 par des enfants ambassadeurs formés lors de sessions de sensibilisation à domicile. Grâce à leur participation à cette initiative, les enfants ont observé et signalé un manque de savon dans la plupart des familles touchées, et ont demandé une distribution supplémentaire de savon pour promouvoir l'hygiène avec le soutien des comités de protection de l'enfance.

Partenaire(s) :

World Vision International

Pour de plus amples informations, contacter :

Véronique Njo : njo@unhcr.org

Brenda Adrako Madrara : Brenda_Madrara@wvi.org; abitimorenda@gmail.com 


\title{
Étude de cas au Soudan du Sud : Mobilisation des étudiants pour la diffusion de messages de prévention de la COVID-19 dans les zones urbaines
}

\begin{abstract}
$\square$ ans le camp de réfugiés de Gorom à Juba, des boursiers du HCR se sont portés volontaires pour aider leurs communautés en partageant des informations sur la prévention de la COVID-19. Les volontaires ont reçu une orientation sur les informations clés de l'Organisation mondiale de la santé en matière de prévention et ont été répartis en équipes dirigées par des agents de santé communautaires ou des promoteurs de l'hygiène. Les équipes mènent des campagnes d'information auprès des ménages, en démontrant les pratiques d'hygiène de base conformément à la documentation IEC recommandée. Les équipes d'étudiants réfèrent également certains cas médicaux particuliers aux partenaires de santé.
\end{abstract}

Partenaire(s) : divers

Pour de plus amples informations, contacter :

Tamar Joanian : joanian@unhcr.org

\section{Pratique émergente au Soudan du Sud : «Tout le monde peut faire la différence ; chaque action compte "}

$\square$

ans le camp de réfugiés de Doro, chaque village a un représentant des jeunes (président) qui siège au Comité des jeunes dudit camp. La population de réfugiés se repartie en huit ethnies différentes, chacune parlant un dialecte qui lui est propre. Sachant qu'il est nécessaire de sensibiliser à COVID-19 et aux bonnes pratiques d'hygiène, cette diversité est utile, car elle permet de communiquer dans les différentes langues locales. Pour réussir, il est nécessaire d'adapter les messages clés de lutte contre la COVID-19 au contexte local du camp.

Dans le cadre de cette intervention dont ils sont le fer de lance, les jeunes du camp de réfugiés ont pris en main la sensibilisation communautaire à la COVID-19. Les présidents d'organisations de jeunes ont composé huit chansons sur la COVID-19 dans leurs langues locales. Les chansons véhiculent les messages sur la COVID-19 qui ont été approuvés par l'Organisation mondiale de la santé. Ce groupe de jeunes se rend dans chacune des communautés, sensibilise les réfugiés à la COVID-19, et chante la version traduite des chansons dans le dialecte parlé dans la composante communautaire concernée.

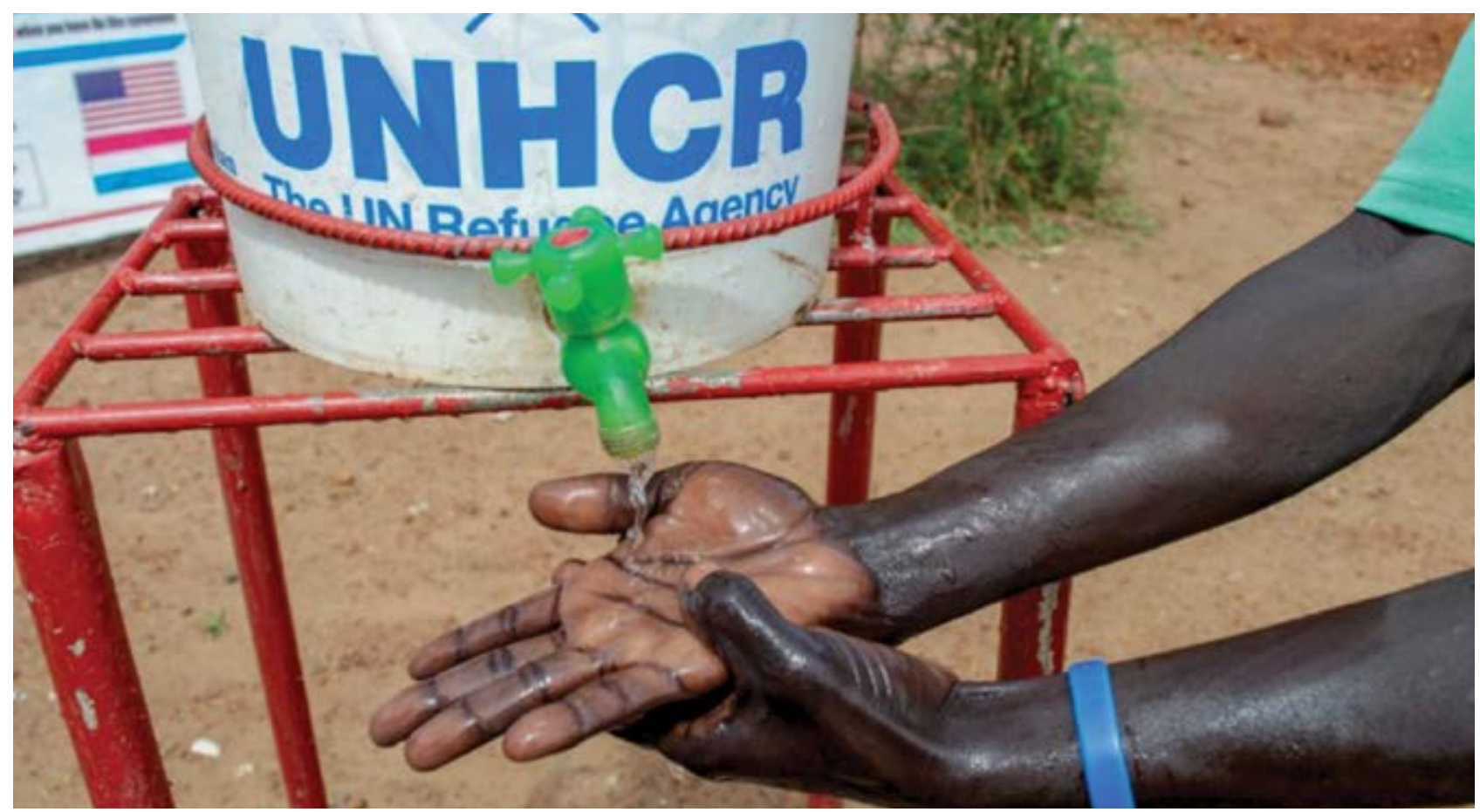



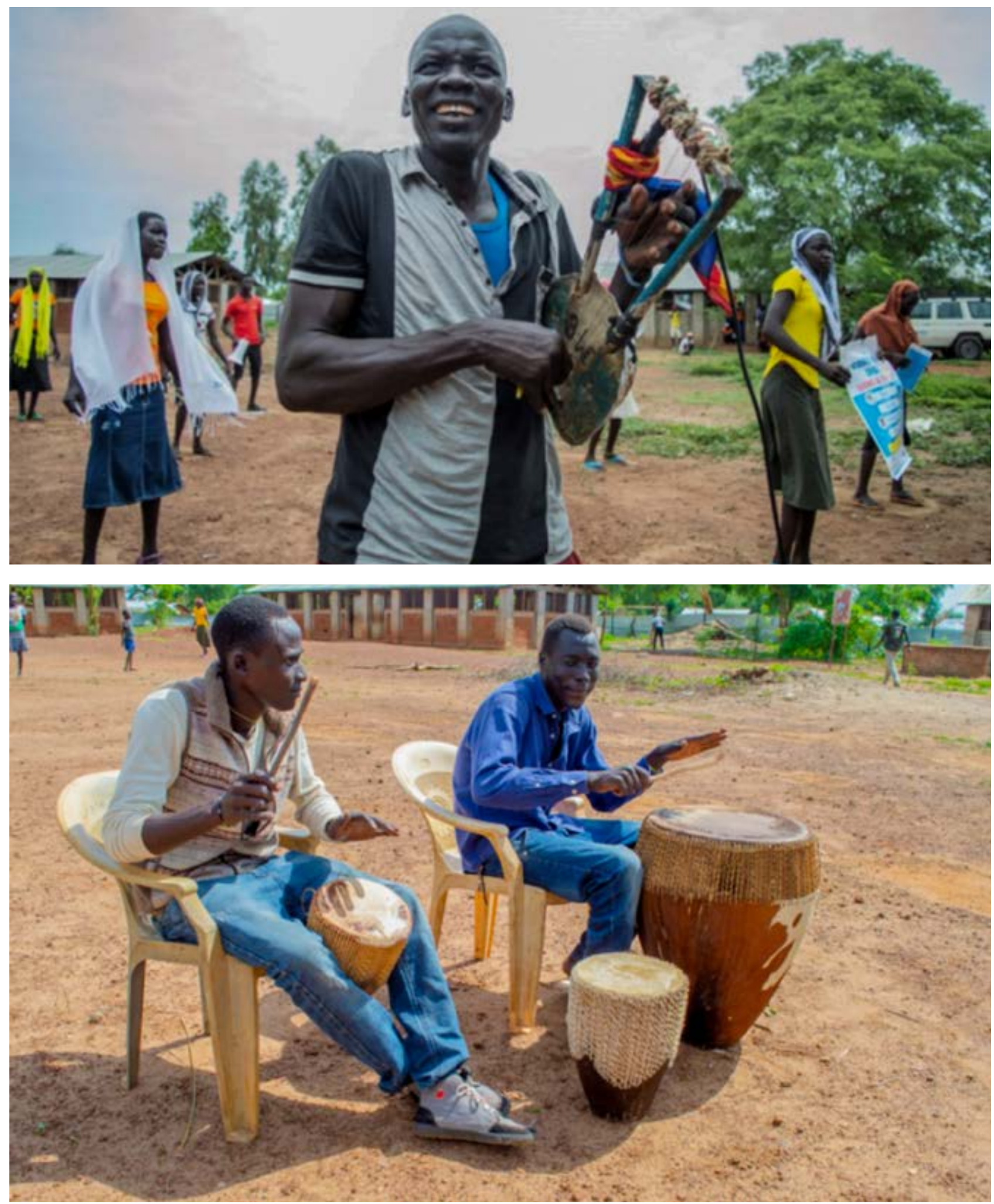

Le groupe assure l'observation de la distanciation physique lors de toutes les représentations ainsi qu'un lavage des mains adéquat.

Lors de chaque événement, une équipe de bénévoles oriente le public vers les postes de lavage des mains. Une grande banderole avec des inscriptions du la COVID-19 est accrochée en arrière-plan des postes de lavage des mains. Lorsque les membres de la communauté font la queue, chacun d'eux doit se tenir à environ un mètre de distance de l'autre. Pour s'assurer qu'ils maintiennent cette distance, les membres de la communauté sont encouragés à se placer au milieu de cercles marqués au sol par du sable. Un bénévole se tient à côté de chaque poste de lavage des mains pour enseigner aux femmes, aux hommes et aux enfants les techniques de lavage des mains recommandées. 


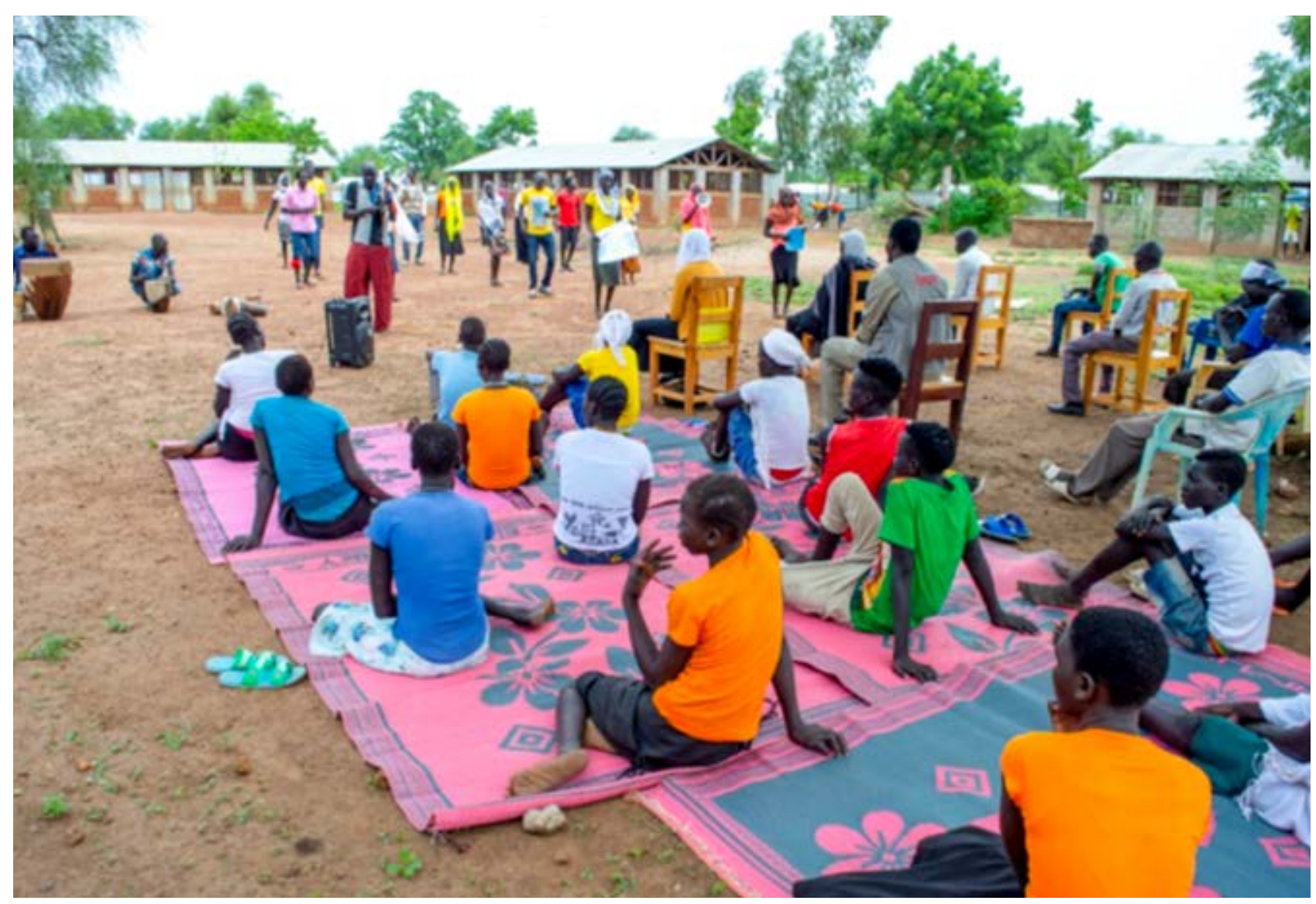

Les chaises destinées au public sont écartées d'un mètre et tous ceux qui choisissent de s'asseoir sur les tapis sont encouragés à maintenir la même distance. Lorsque tous les sièges (chaises et tapis) sont occupés, le spectacle commence. Les chansons englobent des métaphores locales et mettent en avant les bonnes pratiques, telles qu'éviter les poignées de main et se tenir à bonne distance. Après une série de chansons, le groupe organise des échanges sur les différentes mesures de prévention de la COVID-19.

Le Comité de la jeunesse tient un registre du nombre de zones visitées, du nombre de représentations données et du nombre de personnes touchées. En juillet 2020, dans le cadre du programme de lutte contre la violence sexuelle et fondée sur le genre, les structures communautaires de lutte contre cette forme de violence (moniteurs de protection, activistes SASA !, défenseurs des droits des victimes de violence sexuelle et fondée sur le genre) ont participé à des séances de sensibilisation et à la diffusion de messages de prévention ciblant ce fléau, qui ont été intégrés aux messages de prévention de la COVID-19. Cette intervention a permis de toucher au total 4899 membres de la communauté (2 092 hommes et 2807 femmes).

Si ce groupe de jeunes a lancé l'initiative de son propre chef, il n'en demeure pas moins qu'il a besoin du soutien des acteurs humanitaires pour la poursuite de ses activités. Des initiatives locales de ce genre peuvent constituer une plateforme efficace pour transmettre des messages essentiels adaptés au contexte et aux coutumes locales.

\section{Partenaire(s) :}

Commission for Refugee Affairs

Conseil danois pour les réfugiés

\section{Pour de plus amples informations, contacter :}

Dr Gebrewold Petros Yohannes : petros@unhcr.org

Thomas John Hither, Président du Comité de la jeunesse, Alor Kuol, directeur adjoint, Camp de réfugiés de Doro 


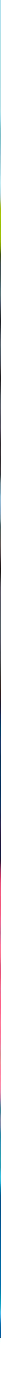

\section{PROTECTION COMMUNAUTAIRE}

\section{Pratique émergente à Djibouti : Services communautaires à distance}

es mesures de confinement dans le cadre de la prévention de la COVID-19 à Djibouti ont conduit le personnel du HCR et les partenaires d'exécution à adopter le télétravail. Cela a eu des répercussions sur l'accès aux services de protection — par les personnes relevant de la compétence du HCR. Lintervention en cours est née de la nécessité de maintenir la continuité des services de protection et l'engagement auprès des réfugiés et des demandeurs d'asile. Elle consiste en la mise en place d'une ligne d'assistance téléphonique pour les réfugiés et les demandeurs d'asile. La ligne est complétée par deux groupes WhatsApp dans les camps de réfugiés - l'un pour les responsables de communauté, l'autre pour les agents de santé communautaires.

Le numéro de téléphone de la ligne d'assistance fait d'une large diffusion (par le biais d'affiches d'information en oromo, somali, français, arabe et anglais) pour informer un large éventail de réfugiés et de demandeurs d'asile (femmes, hommes, filles et garçons) de son existence. La ligne est disponible tous les jours de $8 \mathrm{~h}$ à $17 \mathrm{~h}$, tandis que les groupes WhatsApp fonctionnent en permanence. Les responsables des réfugiés bénéficient de crédit téléphonique prépayé/temps d'utilisation du réseau pour maintenir le contact avec les membres de la communauté, le personnel de protection du HCR et entre eux.

Les personnes ayant des problèmes de santé et d'autres besoins urgents sont orientées par les responsables de communauté vers les travailleurs de la santé, qui offrent des soins et des références de soutien aux partenaires concernés (homologues nationaux ou partenaires d'exécution du HCR) conformément aux mesures de sécurité en place. Les agents de santé communautaires suivent une formation sur la manière de traiter les questions de santé liées à la COVID-19. Les appels entrants sur la ligne d'assistance sont consignés, tout comme les services fournis en réponse à ces appels. 
L'intervention a aidé le HCR à maintenir un contact permanent avec les structures communautaires ; à poursuivre les activités de gestion des cas et à écouter et guider les réfugiés et les demandeurs d'asile sur la base de leurs doléances et face aux problèmes exacerbés auxquels ils sont en proie en raison de la pandémie.

Partenaire(s) : s. o.

Pour de plus amples informations, contacter:

Laura De Somer : desomer@unhcr.org

DrTongna Alain Rodrigue Zoure : zoure@unhcr.org

Oubah Abdi Hassan : abdihaso@unhcr.org

\section{Étude de cas en Éthiopie : Groupes WhatsApp des responsables des communautés de réfugiés et des bénévoles chargés de la sensibilisation des réfugiés à Addis-Abeba}

3 ien que les réfugiés en Éthiopie se trouvent dans des camps de réfugiés, divers groupes résident également en zone urbaine à Addis-Abeba. Certains réfugiés se rendent à Addis-Abeba sur recommandation à partir des camps de réfugiés pour des raisons de santé, de sécurité, de violence sexuelle et fondée sur le genre et d'autres raisons humanitaires qui ne peuvent être y traitées. Les érythréens constituent le plus grand groupe de réfugiés urbains. Un bon nombre réside à Addis-Abeba dans le cadre de la politique « hors camp », un arrangement spécial avec le gouvernement éthiopien qui tient compte de la culture et du contexte historique et des liens entre l'Éthiopie et l'Érythrée.

Diverses mesures de prévention de la COVID-19 introduites à Addis-Abeba, y compris la fermeture du centre d'accueil et la limitation des services personnalisés, ont entravé la communication du HCR avec les communautés de réfugiés résidant à Addis-Abeba. La capitale de l'Éthiopie dispose généralement d'une bonne couverture de réseau de téléphonie mobile et Internet, et les réfugiés qui y sont installés bénéficient d'une connexion réseau de bonne qualité. Jusqu'à $94 \%$ des ménages de réfugiés à Addis-Abeba ont accès à un téléphone portable.

Étant donné que les canaux de communication conventionnels avec les communautés de réfugiés (centre d'accueil, rencontres) sont à présent très limités par les restrictions entrant dans le cadre de la lutte contre la COVID-19, d'autres canaux de communication efficaces ont été explorés. Compte tenu de la bonne couverture de réseau de téléphonie mobile à Addis-Abeba, des canaux de communication numérique ont été mis en place, notamment les groupes WhatsApp du HCR avec les responsables des communautés de réfugiés et les bénévoles de proximité.

Cette intervention visait à assurer une communication rapide et efficace dans les deux sens entre le HCR et les communautés de réfugiés sur un large éventail de sujets, y compris les informations sur la réinstallation, les messages du représentant du HCR aux réfugiés, la disponibilité des services, les numéros de téléphone de la ligne d'assistance, les informations sur les mesures de prévention de la COVID-19 et la mise au point concernant les fausses informations qui circulent.

Le HCR a créé trois groupes WhatsApp pour les responsables des communautés de réfugiés (15 à 20 membres par groupe), représentant toutes les principales nationalités à Addis-Abeba, et un groupe WhatsApp pour les bénévoles chargés de I'aide aux réfugiés. Le personnel du HCR (l'équipe urbaine) tient le rôle d'administrateur des quatre groupes, en publiant des informations pertinentes dans les groupes et en répondant aux questions et aux requêtes.

Les membres des groupes WhatsApp sont tenus de respecter certaines directives, notamment de ne pas discuter de cas, ni de partager des informations sensibles ou personnelles sur les réfugiés.

Les groupes WhatsApp permettent l'accès à des informations pertinentes pour les responsables de réfugiés qui sont à leur tour chargés de les diffuser auprès de leurs communautés, y compris auprès des groupes ayant des besoins spécifiques. Cette communication bidirectionnelle est un outil pour renforcer l'autonomisation et favoriser un partenariat horizontal entre les responsables des réfugiés et le HCR.

Partenaire(s) : s. O.

Pour de plus amples informations, contacter :

Ota Hlinomaz : hlinomaz@unhcr.org

Nunu Aliyi : aliyi@unhcr.org 


\section{Étude de cas au Kenya : Comité de protection communautaire}

a pandémie de la COVID-19 a renforcé la nécessité pour les personnes relevant de la compétence du HCR d'avoir accès à des informations opportunes, pertinentes et exactes dans les langues applicables, tout en garantissant l'accessibilité de la communication et de la gestion des cas de protection critique pour les personnes à risque accru (par exemple, les personnes âgées, les enfants, les jeunes, les personnes handicapées, les LGBTI et les minorités ethniques et religieuses). La communauté a donc proposé la création d'un comité qui travaillerait aux côtés des comités sectoriels. En réponse à cette proposition, et en s'inspirant de l'optique d'âge, de genre et de diversité, le HCR a créé un comité de protection de 36 membres formés aux principes de base de la fourniture de protection, de la confidentialité, de la voie de référence et de la prévention/atténuation de la pandémie de la COVID-19.

Au début de la pandémie, la présence dans les camps du HCR et de ses partenaires a été réduite, bien que le travail se soit poursuivi à distance par le biais de lignes d'assistance, de SMS groupés, de plateformes WhatsApp et d'émissions radiophoniques. Cependant, les réfugiés souhaitaient obtenir de plus amples informations auprès du HCR, arguant qu'ils ne pouvaient pas compter uniquement sur les responsables des réfugiés pour leur fournir des informations.

La principale composante de l'intervention consiste à tirer parti des réseaux existants de bénévoles de proximité et des groupes de femmes, d'enfants et d'autres groupes formés et dirigés par des personnes relevant de la compétence du HCR pour fournir des informations essentielles sur les activités sectorielles essentielles et les besoins de protection, ainsi qu'à veiller à ce que les voies de référence soient maintenues tout en fournissant une protection et un soutien psychosocial au niveau de la communauté. Le Comité de protection fournit une plateforme au HCR pour exploiter et tirer parti de ces réseaux. Ce comité de 36 membres reçoit des informations des partenaires et les transmet à la communauté au niveau du bloc. Le comité travaille également avec les forces de l'ordre et l'agence de gestion du camp pour s'assurer que tous les nouveaux arrivants sont placés dans le centre de quarantaine, ainsi qu'avec d'autres comités sectoriels de réfugiés.

Les autres fonctions du comité sont:

- Effectuer du porte à porte/visites à domicile auprès des familles et fournir des informations de base sur les services offerts et d'autres questions émergentes.

- Identifier en toute sécurité les réfugiés les plus menacés qui ont besoin d'une aide et d'un soutien urgents et les orienter vers le HCR et ses partenaires ou vers les unités/secteurs concernés pour une intervention immédiate.

- Établir des réseaux avec des bénévoles, des travailleurs et des structures de proximité, des chefs de section et de bloc, la police kényane pour faciliter les références.

- Procéder à un échange d'informations au niveau communautaire sur la protection et la prévention contre la contamination par le coronavirus.

- Effectuer un suivi régulier de la protection par section par l'intermédiaire des chefs de section afin de pouvoir recueillir des informations et informer rapidement sur les questions émergentes telles que la santé, la violence sexuelle et fondée sur le genre, la protection des enfants, le logement, les besoins de base et toute autre intention.

- Fournir des informations quotidiennes et un rapport au HCR tous les jeudis.

Le HCR et le Conseil norvégien pour les réfugiés fournissent du temps de communication par téléphone portable aux membres du comité pour assurer une communication bidirectionnelle et faciliter la fourniture de comptes rendus de situation hebdomadaires.

\section{Partenaire(s) :}

Conseil norvégien pour les réfugiés

Secrétariat des affaires des réfugiés

Pour de plus amples informations, contacter :

Lina Koki : koki@unhcr.org

Nicholas Midiwo : midiwo@unhcr.org 


\section{Étude de cas au Kenya : Utilisation par les animateurs de camp de réfugiés de l'application de messagerie WhatsApp dans le domaine de l'eau, de l'assainissement et de l'hygiène (WASH)}

es restrictions de mouvement pendant la pandémie de la COVID-19 ont conduit le HCR et CARE à s'appuyer fortement sur les animateurs de camp de réfugiés pour assurer le suivi des activités WASH dans les communautés de réfugiés.

Les animateurs surveillent les activités au niveau des forages et dans les blocs communautaires. La pandémie a créé la nécessité d'utiliser une plateforme à distance pour la communication des informations de surveillance, y compris la prise et l'envoi de photographies montrant la progression des activités, et la notification de plaintes urgentes telles que les fuites de robinets et les ruptures de canalisations. L'application de messagerie WhatsApp a été utilisée à cette fin. L'utilisation de cette application a permis de garantir que les plaintes sont signalées et traitées rapidement, et que des rapports d'avancement continuent d'être reçus et enregistrés au bureau WASH de CARE. Les animateurs et les chefs de camp ont des contacts téléphoniques avec les agents WASH de CARE et du HCR et peuvent enregistrer des plaintes à tout moment de la journée et de la nuit via cette application.

Partenaire(s) :

CARE

Pour de plus amples informations, contacter :

Osman Yussuf Ahmed : ahmedos@unhcr.org

\section{Pratique prometteuse au Rwanda : Groupe de travail sur l'hygiène communautaire et effort conjoint de la communauté, y compris des musiciens locaux, dans la prévention de la COVID-19}

a pandémie de la COVID-19 et l'épidémie de gale dans les camps ont conduit à une proposition de réunion de coordination des partenaires pour lancer un groupe de travail sur l'hygiène communautaire à Kiziba, coprésidé par l'équipe de protection communautaire de Karongi avec le soutien des partenaires de la santé et WASH.

Les objectifs spécifiques de cette pratique étaient les suivants : évaluer de manière exhaustive la situation en matière d'hygiène (connaissances, pratiques et infrastructures) dans le camp par la mise au point d'outils d'inspection et d'enquêtes professionnelles faciles à utiliser par les personnes relevant de la compétence du HCR; réhabiliter les installations WASH du camp en partageant les recommandations basées sur les résultats de l'inspection et de la recherche; responsabiliser les mobilisateurs communautaires et leur donner la capacité de surveiller l'hygiène du camp et de promouvoir un comportement hygiénique ; et mobiliser la communauté de réfugiés pour qu'elle maitrise les mesures de prévention de la COVID-19 en invitant ses membres (en particulier les jeunes réfugiés) à être au centre des sessions de sensibilisation communautaire et d'autres activités d'évaluation.

Les principales composantes de l'intervention sont les suivantes:

- Inspection de l'hygiène communautaire : Les formulaires d'inspection de l'hygiène ont été élaborés conjointement par le groupe de travail, et l'équipe a facilité l'inspection de l'hygiène pour les ménages, les établissements scolaires et les projets de cantines scolaires. Des recommandations basées sur les résultats des inspections ont été communiquées au partenaire WASH pour améliorer les installations en la matière avant le début de la pandémie de la COVID-19 au Rwanda.

- Initiative des travailleurs communautaires dans le domaine de la santé et de l'hygiène : Cette initiative a été lancée à Kiziba pour former des groupes composés d'un agent de santé communautaire et de plusieurs agents WASH communautaires dans le but d'effectuer des visites à domicile et des campagnes de sensibilisation.

- La mobilisation communautaire et les efforts conjoints des membres : L'équipe de protection communautaire a invité un groupe de rap et de musique de jeunes réfugiés («DJ MC Orphans »), pour animer une campagne de sensibilisation à la prévention de la COVID-19.

- Placer les réfugiés au centre de la recherche sur la COVID-19 dans le camp de réfugiés : En collaboration avec I'Université de l'équité en matière de santé mondiale (UGHE), et avec l'approbation du Ministère de l'éducation et du Ministère en charge de la gestion des urgences (MINEMA), les jeunes réfugiés de Kiziba ont mené une enquête COVID-19 par téléphone sur les connaissances, attitudes et pratiques (CAP) - la première du genre réalisée dans un camp de réfugiés et facilitée par des jeunes réfugiés. Ces jeunes ont participé de manière significative 
à la conception et à la mise en œuvre du projet, développant par la même occasion leurs compétences et leur expérience en matière de recherche.

\section{Résultats}

- Dans le cadre des actions conjointes de prévention de la COVID-19, la communauté des réfugiés a construit 122 postes de lavage des mains supplémentaires pour promouvoir l'hygiène dans le camp.

- Les résultats de l'enquête CAP ont indiqué que les connaissances relatives à la COVID-19 dans le camp étaient généralement bonnes, et que la majorité des réfugiés de Kiziba reconnaissent que la communication sanitaire communautaire est une source importante d'information. De plus, les réfugiés de Kiziba ont obtenu un score plus élevé à l'enquête CAP en ce qui concerne le lavage des mains (comparé à d'autres comportements d'hygiène), ce qui reflète en partie l'impact positif de l'effort communautaire conjoint pour établir des postes de lavage des mains.

- Les femmes ont obtenu un score inférieur à celui des hommes sur les connaissances liées à la COVID-19, en raison de l'analphabétisme et de l'accès limité au téléphone/à la radio.

\section{Partenaire(s) :}

Africa Humanitarian Action

Alight

Pour de plus amples informations, contacter:

Ruizhi Zhu : zhur@unhcr.org; rwaki@unhcr.org

Speciose Buwamaria : buwamari@unhcr.org

\section{Étude de cas en Ouganda : Mobilisation de la communauté par le biais d'un Conseil de protection des réfugiés pour la prévention et l'intervention contre la COVID-19}

es Conseils de protection des réfugiés ont été créés dans le camp de réfugiés d'Imvepi en 2017 afin de renforcer la mobilisation et la coordination des communautés au sein de la population réfugiée. Les Conseils sont composés de responsables de communauté de réfugiés et leur principale fonction est d'identifier, de prévenir et de répondre aux risques dans leur environnement. II s'agit notamment de systématiser les stratégies d'autoprotection positives existantes, de défendre les intérêts locaux, de sensibiliser aux droits de l'homme, au droit et aux services médicaux, juridiques et psychosociaux, et de promouvoir la participation de différents groupes de personnes âgées aux décisions relatives à la protection.

Grâce aux efforts des Conseils, les partenaires de protection, le gouvernement (cabinet du Premier ministre) et le HCR sont devenus plus réceptifs et plus attentifs aux questions et aux besoins de protection, tandis que les personnes relevant de la compétence du HCR ont été mieux informées et ont pu accéder à des services de protection appropriés au sein de l'établissement. Avec la pandémie de la COVID-19, les Conseils sont utilisés comme mécanisme de prévention et de réponse à la maladie dans les domaines suivants :

- Atténuer les risques ou décourager le recours à des stratégies de protection négatives

- Renforcer les stratégies positives existantes (en particulier, les directives COVID-19 de l'OMS et du Ministère de la santé)

- Soutenir de nouveaux mécanismes positifs de prévention et d'intervention qui ne sont pas conformes à certaines conventions antérieures à la COVID-19 (par exemple, rester à la maison, se laver régulièrement les mains au savon, observer la distanciation physique, porter un masque).

Les Conseils sont le premier point de contact/référence dans l'établissement auprès duquel tous les partenaires obtiennent des informations destinées aux personnes relevant de la compétence du HCR. Elles obtiennent des conseils sur la mise en œuvre des activités pendant les périodes de confinement, et sur la façon d'identifier, d'évaluer et de soutenir les plus à risque/vulnérables. Les Conseils travaillent donc au quotidien avec les partenaires opérationnels et de mise en œuvre, le HCR, d'autres organismes des Nations Unies (PAM, UNICEF, UNFPA, ONU-Femmes, etc.) et le cabinet du Premier ministre pour s'assurer que des services de protection de qualité (protection de l'enfance, violence sexuelle et fondée sur le genre, protection générale, protection juridique et physique, protection communautaire) atteignent les personnes relevant de la compétence du HCR. 
La plupart des partenaires ayant adopté le télétravail pendant COVID-19, la coordination entre eux et la communauté a été améliorée grâce aux Conseils qui fournissent un rapport de situation quotidien au HCR et assurent le suivi des points d'action qui leur sont communiqués. Les Conseils ont également contribué à une communication efficace avec les communautés de réfugiés afin d'atténuer la résistance aux nouvelles réglementations suscitées par la COVID-19 (par exemple, les nouvelles directives de distribution alimentaire et les directives nationales de prévention de la COVID-19).

En outre, le rôle de chef de file des Conseils dans les activités communautaires a eu des effets positifs. Par exemple, ils ont réussi à promouvoir une approche de travail communautaire dans laquelle les tâches ne sont pas rémunérées. Les communautés participent également à l'exécution de tâches destinées à inciter les travailleurs à accélérer les efforts de distribution et à éviter les grands rassemblements. Cela est évident lors de la distribution de nourriture, de matériel de gestion de l'hygiène menstruelle et d'événements communautaires tels que les funérailles.

Les conseils jouent également un rôle essentiel dans la liaison avec la communauté d'accueil, en résolvant de manière indépendante les conflits liés à la terre, aux animaux errants, au bois de chauffage et aux poteaux pour la construction d'abris. Ils engagent le dialogue de coexistence pacifique avec la communauté d'accueil, n'impliquant le cabinet du Premier ministre et le HCR que comme témoins des points d'action convenus.

La construction d'abris pour les personnes ayant des besoins spécifiques dans la zone d'installation pendant la pandémie a été aussi réalisée par les Conseils qui ont impliqué la communauté des réfugiés dans cette entreprise. Par exemple, dans la zone 2 (village 14), sous l'impulsion d'une femme du Conseil, des jeunes ont été mobilisés pour réparer cinq abris au profit de personnes ayant des besoins spécifiques pendant la pandémie.

\section{Partenaire(s) :}

Conseil danois pour les réfugiés

Cabinet du Premier ministre

Partenaire d'exécution du HCR

Pour de plus amples informations, contacter :

Willian Ngeze : ngeze@unhcr.org

Faith Kamoyo : kamoyo@unhcr.org

\section{EPratique émergente en Ouganda : Coalitions de soins communautaires}

es personnes ayant des besoins spécifiques, telles que les personnes handicapées, les personnes souffrant de graves problèmes de santé, les femmes en danger, les personnes âgées et les enfants non accompagnés dans les camps de réfugiés, ont des difficultés à accéder aux services, ainsi qu'à la nourriture, à la santé, à l'éducation, à l'eau et à l'aide au logement. Ils sont également souvent plus sensibles aux formes graves de la COVID-19. En raison du nombre limité de personnel de protection communautaire sur le terrain du fait de la COVID-19, une lacune est apparue dans l'identification, l'évaluation et l'orientation des personnes ayant des besoins spécifiques vers les prestataires de services concernés. L'approche des Coalitions de soins communautaires, décrite ci-dessous, vise à améliorer la prestation de services et le soutien aux personnes ayant des besoins spécifiques.

Les ressources consacrées à la protection des personnes ayant des besoins spécifiques dans les opérations à Adjumani étant limitées et en diminution, il était nécessaire d'adopter une approche davantage communautaire pour répondre aux besoins de cette population. Grâce à un accord de partenariat de projet avec la Fédération luthérienne mondiale (FLM), une approche de coalition de soins communautaires a été mise sur pied. Cette approche est mise en œuvre par une structure communautaire appelée «Équipes de la Coalition de soins communautaires ». Elles sont formées en consultation avec les dirigeants du Conseil de protection des réfugiés (responsables des réfugiés dans les différentes camps). Les restrictions nationales entrant dans le cadre de la lutte contre la COVID-19 ont inclus l'interdiction de transport (sauf pour les services essentiels), la fermeture des frontières, la distanciation physique, l'interdiction des rassemblements de masse et une présence de 30 \% sur le lieu de travail. Dans ces circonstances, les équipes de la coalition jouent un rôle essentiel en soutenant les personnes ayant des besoins spécifiques et en veillant à ce qu'ils aient accès aux services et à l'assistance malgré le nombre limité de personnel sur le terrain.

\section{Composantes principales :}

Les principales composantes de cette approche sont les suivantes: 
- Cartographie institutionnelle préliminaire

- Formation du personnel de la protection communautaire à l'approche (pour la mise en œuvre de l'intervention)

- Réunions des acteurs communautaires

- Création d'une Équipe de la coalition

- Planification des actions

- Formation de l'Équipe de la coalition

- Identifier les personnes ayant des besoins spécifiques dans les camps et les mettre en contact avec la structure

- Former les aidants des personne ayant des besoins spécifiques

- Soutenir l'Équipe de la coalition

- $\quad$ Suivi et rapports

- Mobiliser des ressources pour aider à la mise en œuvre des activités communautaires des Équipes de la coalition

À ce jour, 129 réfugiés (66 femmes et 63 hommes) sont membres d'une Équipe de la coalition Les membres de l'Équipe de la coalition portent des tabliers distinctifs pour une identification facile par la communauté. Plusieurs personnes ayant des besoins spécifiques ont été identifiées et évaluées. La communauté connaît mieux les services de proximité et les points d'assistance pour les personnes ayant des besoins spécifiques grâce aux formations dispensées, et la voie de référence vers ces services/assistance a été renforcée en impliquant les équipes de la coalition pour jouer le rôle de guides des personnes ayant des besoins spécifiques dans la zone d'installation. La participation des responsables des réfugiés aux réunions de lancement et de retour d'information trimestrielles a permis une intervention bien coordonnée. En fin de compte, la capacité de la communauté à répondre aux personnes ayant des besoins spécifiques dans le contexte d'un soutien limité a été renforcée.

Partenaire(s) :

Fédération luthérienne mondiale

Pour de plus amples informations, contacter:

Elvira Ndanga : ndangael@unhcr.org

Elizabeth Kaboyo : Elizabeth.kaboyo@lutheranworld.org

Ajavu Patrick : patrick.ajavu@lutheranworld.org

Deogratias Acidri : acidri@unhcr.org

Prosscovia Achiro : achiro@unhcr.org

\section{Pratique émergente en Ouganda : Aide au logement pour les personnes ayant des besoins spécifiques}

a fermeture temporaire du centre d'accueil de Kabazaana en raison de la COVID-19a occasionné l'installation d'urgence de toutes les personnes concernées qui résidaient dans le centre. (Cela s'est fait dans le respect des exigences de

distanciation et physique). Par conséquent, les personnes particulièrement vulnérables pour lesquelles aucun abri n'avait été construit à l'époque n'avaient nulle part où dormir. Ces personnes n'étaient pas en mesure de se construire des abris. Cette situation a rendu les personnes ayant des besoins spécifiques vulnérables aux risques de protection, ainsi qu'aux risques de problèmes de santé provoqués par le froid et l'humidité.

En guise de solution à ce problème, 74 structures temporaires ont été construites au total, dans lesquelles 65 personnes particulièrement vulnérables et 9 mères célibataires ont été hébergées. Une approche communautaire a été utilisée pour la construction de l'abri, y compris l'apport de matériaux de toiture (bâche plastique fournie par le HCR dans le cadre de son programme d'installation comprenant des articles de secours de base) par des personnes particulièrement vulnérables. Les chefs et relais communautaires ont été chargés de l'évaluation, de la supervision et du suivi de la construction, tandis que les membres compétents de la communauté ont fourni de la main-d'œuvre à un coût réduit, en plus de la protection des biens des personnes particulièrement vulnérables. ALIGHT a contribué à hauteur de 53 dollars par abri pour l'achat de matériaux tels que des poteaux, des roseaux, des clous, le transport et la main-d'œuvre.

\section{Résultats}

L'intervention a enregistré des résultats positifs du point de vue des personnes ayant des besoins spécifiques et des autres membres de la communauté. Lorsqu'on lui a demandé son avis sur l'aide au logement, un bénéficiaire s'est exprimé en ces termes : «je suis vraiment comblé de posséder une maison où je peux vivre en toute intimité. Même s'ils nous disent de retourner au centre d'accueil après cette pandémie, je ne suis pas prêt à y retourner. Je veux m'installer ici. Merci, 
Alight ». De même, un travailleur communautaire déclare : «j'apprécie l'approche utilisée par Alight, où les membres de la communauté aident d'autres membres à construire ces maisons pour les personnes particulièrement vulnérables, et cela va les aider à s'installer rapidement. En ma qualité de travailleur communautaire, je suis heureux de voir mon peuple soutenu par Alight en cette période où la pandémie nous a tous touchés».

\title{
Partenaire(s) :
}

Alight

Pour de plus amples informations, contacter :

Davis Mugumya : mugumya@unhcr.org

Sylvia Samanya : samanya@unhcr.org

\section{Étude de cas au Soudan du Sud: Équipes spéciales COVID-19 communautaires}

\begin{abstract}
A fin d'éviter la propagation de la COVID-19 dans les camps de réfugiés d'Ajoung Thok et du Pamir, les services qui impliquaient des rassemblements de masse et des contacts physiques ont été réduits. Les bureaux extérieurs du HCR ont suspendu les visites sur de terrain et l'engagement physique de la communauté. Cela a laissé un vide dans la diffusion rapide et efficace habituelle de l'information à la communauté sur la COVID-19 et des mesures préventives. L'enregistrement des réfugiés a également été affecté, les activités connexes étant menées à distance. Si la sensibilisation s'est faite par la radio, l'analyse des réactions sur le terrain a montré que de nombreux réfugiés avaient encore des questions à poser.
\end{abstract}

En résumé, il était nécessaire de communiquer efficacement sur les activités communautaires dans le cadre des directives liées à la COVID-19; d'identifier et d'orienter rapidement les personnes ayant des besoins spécifiques et les enfants à risque ; et de mener des campagnes d'information sur les nouvelles modalités d'enregistrement à distance. Une intervention a dès lors été mise au point pour sensibiliser à la COVID-19, suivre les rumeurs sur la maladie dans la communauté, et identifier et orienter les personnes ayant des besoins spécifiques vers les services requis pendant la période de pandémie.

Plus précisément, des équipes spéciales COVID-19 ont été formées par les responsables des conseils de réfugiés dans les deux camps, dans le but de fournir un soutien au HCR et à ses partenaires dans la promotion d'une communication à double sens avec la communauté des réfugiés, et produire des informations sur leurs besoins (en particulier ceux des personnes ayant des besoins spécifiques pendant la COVID-19), et sur les mesures de précaution mises en œuvre. À Ajuong Thok, l'équipe spéciale COVID-19 communautaire est composée de 16 membres (4 femmes et 13 hommes). Dans le Pamir, l'équipe spéciale est composée de 10 membres ( 5 femmes et 5 hommes). Les deux équipes spéciales sont présidées par le président du camp.

Les équipes spéciales COVID-19 dirigées par les communautés soutiennent également le HCR dans l'enregistrement à distance en recensant les informations individuelles sur les nouveau-nés dans leurs communautés. Elles travaillent en collaboration avec les partenaires de la santé pour identifier les réfugiés qui retournent dans les deux camps afin que les partenaires de l'hygiène puissent les surveiller et recevoir immédiatement les informations sur la COVID-19. IIs signalent également les cartes de rationnement inactives, et les cartes de rationnement perdues qui doivent être remplacées et réactivées au service de protection du HCR au nom des réfugiés. Les équipes spéciales participent aux réunions hebdomadaires du Conseil des réfugiés, auxquelles assistent également le HCR, le Conseil danois pour les réfugiés et les services de protection de l'enfance de la FLM. Le Conseil danois pour les réfugiés apporte un appui aux équipes spéciales dans les deux camps en mettant à leur disposition des mégaphones, des masques faciaux, des rafraîchissements et de la papeterie afin de mener efficacement des sessions de sensibilisation au sein de la communauté.

Partenariat(s) :

Conseil danois pour les réfugiés

Fédération luthérienne mondiale

Pour de plus amples informations, contacter :

Ivy Wahome : wahomei@unhcr.org

Regina KhamisWude:wude@unhcr.org

Abraham Mijok Ayuel Dau : dau@unhcr.org 


\section{Étude de cas au Soudan du Sud : Production de masques}

e bureau extérieur du HCR à Malakal a commencé la production de masques par les personnes déplacées et les communautés d'accueil dans le cadre d'une initiative de subsistance et d'une activité de consolidation de la paix

-visant à atténuer l'impact de la COVID-19. La sous-délégation de Jamjang étudie la possibilité d'engager les anciens élèves des centres de formation professionnelle (tant les réfugiés que les membres des communautés d'accueil) pour produire des masques réutilisables une fois que la disponibilité du budget sera confirmée.

Partenaire(s) : s. o.

Pour de plus amples informations, contacter :

ViTran : tran@unhcr.org

\section{Étude de cas au Soudan du Sud: Station de radio communautaire}

$\int$ ans les camps de réfugiés de Jamjang, le HCR a travaillé avec la radio Jamjang FM pour diffuser des informations sur la prévention de la COVID-19. Différentes modalités de messagerie sont utilisées, y compris des annonces et des tribunes radiophoniques. En outre, certains réfugiés élaborent actuellement un sketch qui sera diffusé sur la station de radio FM et qui comprendra d'importants messages de prévention et d'intervention contre la COVID-19. Dans certaines émissions, le HCR et les médecins partenaires d'exécution ont été engagés pour aider à briser les mythes. Des plans sont en cours pour diffuser un programme qui répondra aux questions, aux rumeurs et aux idées fausses qui circulent dans la communauté autour de la COVID-19.

II est nécessaire de veiller à ce que davantage de personnes concernées aient accès à des postes de radio afin d'améliorer leur exposition aux messages. Les appareils préférés sont les radios de téléphone portable, car ils sont polyvalents et peuvent servir à la fois de téléphones portables pour l'engagement et les réactions de la communauté, et de postes de radio pour recevoir des informations importantes de santé publique diffusées par le Ministère de la santé du Sud-Soudan ainsi que par le HCR et ses partenaires par l'intermédiaire de Jamjang FM.

Des efforts sont en cours pour assurer la réciprocité de la communication entre les personnes relevant de la compétence du HCR et les acteurs de la santé publique qui diffusent des informations sur la COVID-19 par la voie des ondes. Des évaluations ponctuelles des réactions de la communauté ont été menées principalement par le personnel de santé qui s'est renseigné auprès des membres de la communauté qui fréquentent les centres de santé. La communauté apprécie les messages et reçoit des informations précises diffusées par la radio.

Partenaire(s) :

Jamjang FM

Pour de plus amples informations, contacter :

Tamar Joanian : joanian@unhcr.org

\section{Pratique émergente au Soudan du Sud : Coordination humanitaire pour la réponse à la COVID-19}

e groupe de travail de Maban sur la communication des risques et l'engagement communautaire (CREC) a été cité à de nombreuses reprises par le groupe de travail technique national CREC du Sud-Soudan comme un exemple de «meilleure pratique de collaboration interinstitutions sur le terrain ». Les partenaires humanitaires n'ont mis en place aucun mécanisme de coordination CREC similaire en réponse à la pandémie de la COVID-19 ailleurs au Sud-Soudan. Au début de la COVID-19 dans le pays, les partenaires humanitaires à Maban ont activé le groupe de travail CREC de Maban dans le but d'harmoniser leur approche des interventions de communication sur les risques liés à la COVID-19. Le groupe 
de travail CREC est piloté conjointement par le gouvernement (département de la santé du comté de Maban) et le HCR. Au total, 21 partenaires humanitaires se sont volontairement inscrits pour être membres du groupe de travail CREC de Maban, qui se réunit toutes les deux semaines.

Le groupe de travail CREC de Maban comprend quatre sous-groupes : 1) un sous-comité de suivi et de gestion des rumeurs sur la COVID-19, 2) un comité de pilotage des comportements face à la COVID-19, 3) une unité de suivi, évaluation, responsabilisation, recherche et apprentissage, et 4) un sous-groupe de travail sur la santé mentale et le soutien psychosocial. Les tâches de chaque groupe sont décrites ci-dessous.

Sous-comité de suivi et de gestion des rumeurs sur la COVID-19 : Dirigé par Internews avec le concours du Conseil danois pour les réfugiés.

- Formation de formateurs à l'intention de tous les partenaires humanitaires à Maban sur le suivi et la gestion efficaces des rumeurs, de la désinformation et des préoccupations.

- Formation en cascade à l'intention des organismes partenaires chargés de la mise en œuvre.

- Mise au point d'un outil interinstitutions de suivi en ligne harmonisé, que tous les partenaires de Maban utilisent pour signaler les rumeurs.

- Production périodique d'un rapport sur la gestion des rumeurs qui recense les rumeurs circulant au sein de la communauté, ainsi que les réponses prévues. Le rapport est transmis au sous-comité national de gestion et de suivi des rumeurs.

- Développement d'un parcours de gestion des rumeurs qui garantit que le retour d'information sur le rapport de gestion des rumeurs parvient à la communauté.

\section{Comité de pilotage des comportements face à la COVID-19 : Dirigé par Save the Children International avec le concours de Relief International.}

- Fourniture de conseils fondés sur des données probantes aux autorités locales et aux partenaires sur les stratégies de changement de comportement à court et à long terme pour assurer l'adoption des comportements de prévention de la COVID-19 chez les réfugiés.

- Fer de lance de deux campagnes de sensibilisation sur la COVID-19 et de deux projets de recherche, associés à un soutien aux partenaires humanitaires pour documenter les histoires des agents de changement à l'ère de la pandémie.

\section{Unité de suivi, évaluation, responsabilisation, recherche et apprentissage (MEARL) : Dirigé par le Programme alimentaire mondial avec le concours du Service jésuite des réfugiés et le HCR.}

- Collecte de données de suivi du CREC

- Les partenaires humanitaires à Maban soumettent leurs données de suivi CREC à l'unité MEARL sur une base hebdomadaire. Ces informations sont consolidées et soumises chaque semaine au groupe de travail technique national du CREC. L'unité MEARL partage également des rapports d'analyse de données exploratoires avec les partenaires humanitaires, en plus des données brutes du CREC.

- Suite à une réunion de sensibilisation du groupe de travail CREC de Maban visant à promouvoir le partage des données entre les partenaires humanitaires, l'unité MEARL a organisé une formation interinstitutions pour tous les points focaux de suivi et évaluation dans le comté de Maban, renforçant ainsi leur capacité à utiliser l'outil de suivi harmonisé.

- Soutenir les initiatives de recherche interinstitutions du CREC

- L'unité MEARL a aidé le comité de pilotage des comportements à mener deux projets de recherche interinstitutions du CREC : 1) une enquête rapide sur les connaissances, les attitudes et les pratiques qui a été menée dans les camps de réfugiés et la communauté d'accueil ; et 2) une recherche sur la manière d'impliquer plus efficacement la communauté dans les interventions COVID-19. Les deux projets de recherche ont bénéficié du soutien du groupe de travail technique national du CREC et ont façonné les politiques d'orientation du CREC au Sud-Soudan.

\section{Sous-groupe de travail sur la santé mentale et le soutien psychosocial (SMSPS) : Dirigé par le Service jésuite des réfugiés et conjointement par le Conseil danois pour les réfugiés .}

- Rationalisation de la SMSPS dans le cadre de la réponse à la COVID-19 en veillant à ce que les considérations connexes soient harmonisées et intégrées dans les messages clés et le partage d'informations entre les partenaires et les structures communautaires dans les communautés d'accueil et de réfugiés; renforcement de la composante SMSPS dans les structures en place dans la communauté d'accueil et les camps de réfugiés ; soutien et renforcement des capacités en matière de SMSPS (comme les premiers secours psychologiques) pour les travailleurs de première ligne, les partenaires et les structures communautaires; promouvoir l'intégration des personnes ayant des besoins spécifiques dans les activités de SMSPS; consolider les informations sur la situation 
du bien-être en matière de SMSPS ainsi que la prestation de services pour soutenir l'analyse des lacunes, la réponse coordonnée et le plaidoyer pour l'allocation de ressources aux partenaires de SMSPS ; maintenir une communication et un contact réguliers avec le groupe de travail technique national sur la SMSPS, y compris la participation aux réunions du groupe travail technique national sur la SMSPS ; et promouvoir l'apprentissage mutuel et l'échange d'idées et de meilleures pratiques entre les partenaires.

- Le sous-groupe de travail SMSPS de Maban est représenté au sein du groupe de travail national sur la SMSPS.

\author{
Partenaire(s) : \\ Conseil danois pour les réfugiés \\ Internews \\ Service jésuite des réfugiés \\ Maban County Health Department \\ Relief International \\ Save the Children International \\ Programme alimentaire mondial
}

Pour de plus amples informations, contacter:

Dr Gebrewold Petros Yohannes : petros@unhcr.org

\title{
Étude de cas au Soudan du Sud: Engagement communautaire par la traduction des messages sur la COVID-19
}

es enseignants de la communauté du Kordofan aident à traduire les messages de prévention de la COVID-19 dans les principales langues locales des réfugiés pour les diffuser avec l'aide des dirigeants locaux. Cette initiative vise à sensibiliser les gens et à réduire la transmission de la COVID-19. Dans certains endroits, comme à Khartoum, les communautés ont joué un rôle actif dès les premières étapes de la réponse à la COVID-19, en diffusant des affiches d'information et en identifiant des emplacements pour elles dans leur zone par l'intermédiaire des bénévoles, des chefs communautaires et religieux et des sultans.

Partenaire(s) : s. o.

Pour de plus amples informations, contacter :

Annheli Aldhammar : aldhamma@unhcr.org

\section{Étude de cas au Soudan du Sud : Engagement communautaire par téléphone portable}

$\square$ ans l'État du Nil Blanc et dans d'autres régions, les responsables des communautés de réfugiés et d'accueil ont été sélectionnés et formés sur la voie de référence pour la COVID-19 afin de renforcer leur capacité à répondre aux cas de manière rapide et efficace. Ils ont également bénéficié d'un crédit téléphonique (temps de communication par téléphone portable) pour faciliter la communication. À Khartoum, le HCR soutient un comité local de santé composé de personnes relevant de la compétence du HCR qui travaille avec le Ministère de la santé pour surveiller les symptômes liés à la COVID-19 chez les membres de la communauté. Le HCR Soudan a également lancé un «schéma de communication WhatsApp » principalement pour les communautés de réfugiés érythréens, syriens et yéménites à Khartoum. Cela permettra de diffuser des informations via WhatsApp au sein d'un réseau de groupes de réfugiés, facilitant ainsi la diffusion opportune d'informations auprès des communautés et le retour d'informations de la part de celles-ci.

Partenaire(s) : s. o.

Pour de plus amples informations, contacter :

Annheli Aldhammar : aldhamma@unhcr.org 


\section{VIOLENCE SEXUELLE ET FONDÉE SUR LE GENRE/ATTÉNUATION DES RISQUES/RÉPONSE}

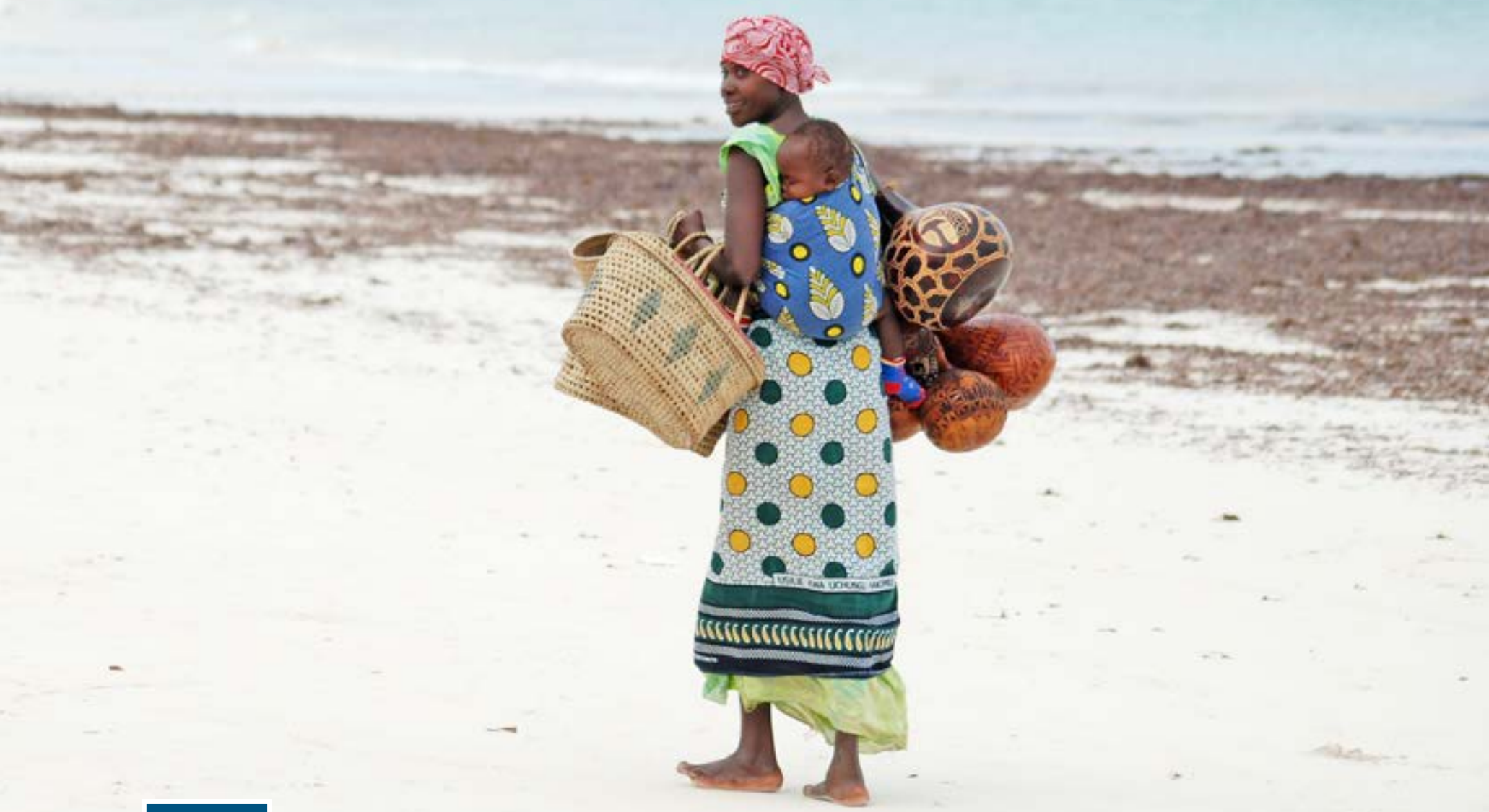

\section{3}

\section{Étude de cas au Burundi : Orientation et gestion par téléphone portable des cas de violence sexuelle et fondée sur le genre}

M

algré les restrictions de mouvement au Burundi durant la pandémie de la COVID-19, le HCR a pu assurer la continuité des soins pour les survivants de violence sexuelle et fondée sur le genre, et la mise en contact pour les soins au profit des survivants nouvellement identifiés en délivrant des téléphones portables et du temps de communication sur une base mensuelle aux acteurs clés de la protection communautaire dans les camps de réfugiés. Au nombre des acteurs on compte les chefs de communauté, les agents de sécurité, les membres de la communauté d'accueil du comité mixte de sécurité, ainsi que les présidents et vice-présidents des comités sectoriels. Cette intervention permet à ces acteurs d'alerter les partenaires d'exécution concernés au sujet de cas de violence sexuelle et fondée sur le genre et d'en assurer le suivi. Elle permet également au HCR de transmettre à distance des messages importants aux présidents et vice-présidents des comités sectoriels, qui sont à leur tour transmis à la communauté dans son ensemble. Pour faciliter l'orientation et le suivi des cas de violence sexuelle et fondée sur le sexe, deux facilitateurs par camp sont désignés pour assurer la liaison avec les travailleurs sociaux en cas de besoin. Les cas urgents de violence sexuelle et fondée sur le genre sont référés aux services qui sont disponibles dans le camp pendant la période de pandémie. Lorsque 
la gestion des cas est nécessaire, les facilitateurs contactent par téléphone les travailleurs sociaux, le partenaire d'exécution du HCR en matière de violence sexuelle et fondée sur le genre (Comité international de secours) ou le point focal du HCR en matière de violence sexuelle et fondée sur le genre.

Partenaire(s) : Comité international de secours

Pour de plus amples informations, contacter:

Gwendolyn Roeske : roeske@unhcr.org

Brigitte Mukanga Eno : eno@unhcr.org

Christine Irambona : irambona@unhcr.org

\section{Étude de cas en Ouganda : Formation des structures communautaires à l'identification, au signalement et au suivi des questions de protection}

0 endant le confinement décrété pour endiguer la COVID-19, des cas nécessitant une attention particulière ont continué à émerger des communautés de réfugiés, mais certains dirigeants et structures communautaires n'avaient pas les connaissances appropriées concernant les processus de protection et de rapport liés à ce secteur.

Comme les structures communautaires et les dirigeants du Conseil d'aide aux réfugiés sont logés dans des communautés de réfugiés, cette intervention a cherché à s'appuyer sur ces ressources communautaires pour aider à identifier les cas de protection, y compris les cas de violence domestique ainsi que les cas de maltraitance et d'exploitation des enfants. Outre l'identification des cas, il était nécessaire de les signaler aux partenaires concernés pour qu'ils interviennent.

L'intervention a permis d'outiller ces structures communautaires en les formant à l'identification des problèmes et des risques de protection (violence sexuelle et fondée sur le genre, protection de l'enfance et juridique), ainsi qu'au signalement en temps utile de ces cas en vue d'une intervention. Cette approche a permis de combler les lacunes en matière de protection causées par les restrictions dues à la COVID-19, qui limitaient la présence des partenaires dans la communauté.

Partenaire(s) : HADS

Pour de plus amples informations, contacter:

William Ngeze : ngeze@unhcr.org

Mansur Buga : buga@unhcr.org

\section{Étude de cas au Soudan du Sud : Référence par téléphone portable et séances de soutien psychosocial}

es références par téléphone portable sont utilisées pour atteindre les réfugiés qui ont besoin d'aide, en particulier dans les régions difficiles d'accès des États du Haut-Nil, de l'Unité, de l'Équateur central et de Jonglei. Dans les endroits -où les réseaux mobiles ne sont pas disponibles, les partenaires de santé et les autorités locales se concertent pour servir de points de référence d'urgence. Ils réfèrent par la suite les cas aux équipes de protection et d'autonomisation des femmes du Comité international de secours (IRC) et à la Fédération luthérienne mondiale (FLM) pour une intervention ultérieure, y compris le soutien psychosocial. L'RC et la FLM ont dispensé une formation de base à quelques travailleurs chargés de l'encouragement des réfugiés dans les camps de Jamjang pour leur permettre de fournir un soutien de base pour les cas de violence sexuelle et fondée sur le genre et de protection des enfants en cas de situations de confinement qui restreindraient les mouvements des membres de la communauté.

Partenaire(s) :

Comité international de secours

Fédération luthérienne mondiale

Pour de plus amples informations, contacter:

ViTran : tran@unhcr.org 


\section{PRATIQUES NON LIÉES À LA COVID}

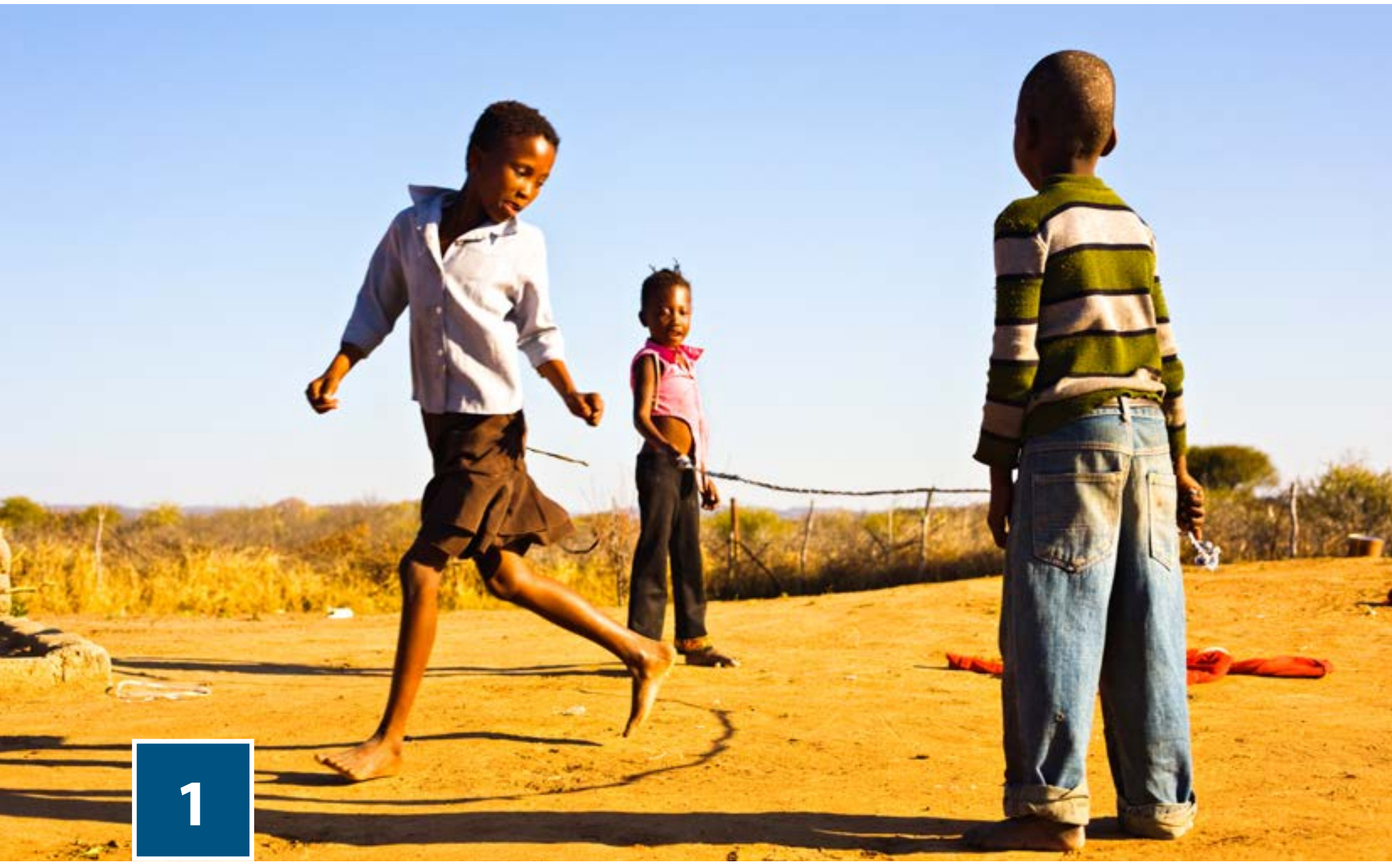

\section{PROTECTION DE L'ENFANCE ET DE LA JEUNESSE}

\section{Pratique prometteuse en Éthiopie : Évaluation et programmation de l'éducation et approche d'engagement communautaire}

a stratégie d'éducation de l'opération du HCR en Éthiopie se concentre sur l'amélioration des résultats d'apprentissage, la garantie que les établissements scolaires sont des environnements d'apprentissage sûrs, l'amélioration de l'accès à

l'enseignement secondaire et supérieur, la garantie que les possibilités d'éducation sont permanentes et disponibles pour tous en fonction des besoins, et l'accès aux possibilités d'éducation le plus tôt possible pendant une situation d'urgence. Le taux de scolarisation est faible pour diverses raisons, notamment les difficultés économiques et la mobilité des réfugiés dans certains camps.

En réponse à cette réalité, l'opération procède à une évaluation conjointe annuelle afin de faire le point de la situation globale des programmes d'éducation, les lacunes et les besoins, et de servir de référence pour la planification de l'année suivante. L'évaluation conjointe a lieu après la fermeture des classes en fin d'année, entre juin et juillet, avec la collecte de données qualitatives et quantitatives pour mesurer les niveaux de rendement par rapport à la planification annuelle. Des questionnaires et des listes de contrôle des observations sont également utilisés. Les participants à l'évaluation conjointe 
comprennent l'Administration pour les réfugiés et les rapatriés (ARRA), la Development and Inter-Church Aid Commission, Save the Children International (SCI), le HCR, le Bureau de l'éducation de Woreda, les enseignants, les associations de parents d'élèves (APE), les représentants des communautés et les dirigeants. Les données secondaires sont tirées des rapports mensuels et de suivi fournis au HCR et aux bureaux de zone de I'ARRA.

Pendant l'évaluation conjointe, il y a un compte rendu des résultats au niveau communautaire, ce qui favorise la participation des représentants de la communauté et la triangulation des résultats. Une approche spécifique d'engagement communautaire est employée dans le cadre de l'intervention, ce qui implique les aspects suivants :

- Participation des représentants de la communauté aux évaluations conjointes : Chefs religieux, associations de femmes, comités centraux de réfugiés et autres membres sélectionnés par la communauté en fonction de leur capacité à transmettre le point de vue de la communauté.

- Suivi de l'assiduité des élèves et des enseignants tout au long de l'année scolaire par les APE. (II y a une APE par établissement scolaire - 43 au total, chacune composée de 7 réfugiés, ainsi que des conseils d'élèves [5 à 8 élèves par conseil d'élèves dans les écoles secondaires] et des délégués d'élèves [3 à 4 dans chaque école primaire]).

- Renforcement des capacités des APE. Par exemple, à la mi-2020, 43 APE avaient reçu une formation sur leurs rôles et responsabilités dans les établissements scolaires.

- L'utilisation de mécanismes de plainte dans les établissements scolaires avec des procédures de suivi efficaces (par exemple, des boîtes à plaintes, des points focaux dans les écoles [dont certains ne traitent que les plaintes de violence sexuelle et fondée sur le genre]).

- Promouvoir la participation des parents à des activités scolaires spécialement conçues, telles que des séances de contes et de chants préscolaires animées par les parents et axées sur le patrimoine culturel. Ces activités ont lieu tout au long de l'année pour renforcer la participation des parents.

L'évaluation conjointe offre une plateforme permettant à la communauté d'exprimer ses préoccupations, de faire des suggestions en rapport avec les programmes et de participer au processus de planification de l'éducation. Les résultats de l'évaluation conjointe annuelle sont documentés et utilisés comme outil de plaidoyer, en plus de servir de référence pour l'allocation des ressources et l'élaboration des propositions.

Il convient de noter que le HCR a mis en place un mécanisme de coordination efficace avec le bureau de l'éducation du district local afin d'intégrer le travail du HCR en matière d'éducation dans le système éducatif national, en renforçant la durabilité, en maintenant les normes nationales et en améliorant l'acceptation. Le rôle de coordination du HCR est d'assurer une réponse efficace et multipartite aux besoins de développement des réfugiés et des communautés d'accueil. Le groupe de travail sur l'éducation, qui comprend des représentants tels que I'ARRA, le HCR, le Programme alimentaire mondial et d'autres partenaires d'exécution (Save the Children International, Development and Inter-Church Aid Commission), est en place et lié par un mémorandum d'accord. II comprend 43 établissements d'enseignement (19 centres de soins et d'éducation de la petite enfance, 21 écoles primaires, 3 écoles secondaires et le College of Teacher Education).

\section{Résultats}

Depuis son adoption en 2015, cette approche a permis d'améliorer la fréquentation et la rétention scolaires. Le taux de fréquentation du cycle primaire était de 83 \% en 2019 et le nombre d'inscriptions au niveau secondaire dans les écoles de réfugiés et de communautés d'accueil a augmenté de $53 \%$ au cours du premier semestre 2020.

\section{Partenaire(s) :}

Administration pour les réfugiés et les rapatriés

Development and Inter-Church Aid Commission

Save the Children International

Bureau de l'éducation de Woreda

Programme alimentaire mondial

Pour de plus amples informations, contacter :

Muhammad Harfoush : harfoush@unhcr.org

Jael Shisanya : shisanya@unhcr.org 


\section{Pratique émergente au Kenya : Approche familiale et communautaire des soins aux ménages dirigés par des enfants}

e placement dans la famille d'accueil n'est pas toujours possible pour les enfants qui ont besoin de ce service dans les camps de réfugiés, en raison de la diversité des cultures et des pratiques en matière de placement. Pourtant, de nombreuses situations dans les camps de réfugiés donnent lieu à la nécessité d'un placement familial, notamment lorsque les enfants sont abandonnés par des parents qui retournent dans leur pays d'origine ; les mauvais traitements infligés à certains enfants placés auprès d'un membre de leur famille élargie, qui nécessitent une séparation à court ou à long terme ; l'absence de placement familial approprié pour les enfants sauvés d'une prise en charge inadéquate ; et l'arrivée de mineurs non accompagnés dans un camp de réfugiés sans que des parents aient été identifiés ou qu'un placement familial approprié ait été trouvé.

L'intervention en cours vise à fournir une structure fiable, dirigée par la communauté, pour des soins durables qui servent d'organe de soutien aux ménages dirigés par des enfants ; à améliorer l'identification sûre des problèmes de protection des enfants vivant dans les ménages dirigés par l'un d'entre eux et le lien avec les interventions de protection appropriées; et à aider les adolescents qui atteigne la limite d'âge à développer des compétences de vie et des capacités d'adaptation dans les camps de réfugiés.

À cette fin, des réunions de retour d'information ont été organisées avec les ménages dirigés par des enfants afin d'offrir un forum de dialogue sur les questions qui les concernent. Les ménages dirigés par des enfants ont été invités à identifier les adultes de leur choix comme mentors/superviseurs auprès de qui ils préféraient obtenir un soutien multiforme. Les mentors identifiés ont été informés de leur sélection par un enfant, et réunis pour des séances de sensibilisation sur les responsabilités parentales et le rôle des mentors/superviseurs dans le cadre de cette initiative, y compris le signalement des problèmes de protection que les enfants peuvent rencontrer.

Les réunions de suivi avec les ménages dirigés par des enfants ont révélé que les mentors/superviseurs soutenaient déjà lesdits ménages de manière proactive sous diverses formes, notamment en allant chercher de l'eau pour les enfants lorsqu'ils sont à l'école, en leur faisant à manger et en les emmenant à l'hôpital lorsqu'ils tombent malades. Cette attitude proactive des mentors/superviseurs a été saluée et encouragée.

Les enfants ayant besoin d'être placés sont identifiés par le biais de divers mécanismes, tels que les structures communautaires, les centres Furaha (guichet unique interinstitutions de protection de l'enfance), pendant les cycles de distribution de nourriture, par le biais de rapports aux bureaux du Conseil danois pour les réfugiés, ou par le HCR par le biais d'un soutien bimensuel sur le terrain ou de référence par d'autres unités. Les recommandations appropriées sont faites par l'unité de protection de l'enfance du HCR, en utilisant les voies de référence interinstitutions établies dans le camp de réfugiés. Une évaluation de l'enfant est effectuée à l'aide d'outils d'évaluation standardisés de l'intérêt supérieur et d'un plan de prise en charge proposé en collaboration avec l'enfant. Les perspectives de soins alternatifs, tels que la parenté et le placement en famille d'accueil, sont d'abord explorées. Le HCR examine les plans de prise en charge, et en l'absence d'un lien de parenté ou d'un placement en famille d'accueil, un accord de vie autonome intermédiaire et supervisé est conclu entre le partenaire et l'enfant. Pour faciliter ce processus, l'enfant communique les noms d'adultes avec lesquels il est déjà à l'aise pour agir en qualité de mentor/superviseur, et ces derniers sont sensibilisés pour soutenir l'enfant comme décrit ci-dessus.

\section{Résultats}

Depuis son lancement, un total de 50 mentors/superviseurs (16 hommes et 34 femmes) ont été identifiés, sensibilisés et mis en relation avec des enfants.

\section{Partenaire(s) :}

Conseil danois pour les réfugiés

Fédération luthérienne mondiale

Pour de plus amples informations, contacter:

Amin Afridi : afridi@unhcr.org

Alexander Novikau : novikau@unhcr.org

Ina Guya : guyai@unhcr.org 


\section{Pratique émergente au Kenya : Structures communautaires de protection de l'enfance}

$\checkmark$ ne série de préoccupations et de risques liés à la protection des enfants sont apparus dans les camps de réfugiés de Dadaab, notamment le travail des enfants, la prostitution par mesure de survie, les grossesses précoces, la séparation des enfants d'avec les personnes qui s'occupent d'eux, l'abandon scolaire, la traite des enfants, le risque de recrutement par les forces armées et le mariage précoce/forcé des jeunes filles. Les risques sont liés à l'insuffisance des capacités financières et à l'insécurité alimentaire, à l'augmentation de la consommation de produits stupéfiants chez les jeunes et à la présence accrue de demandeurs d'asile non enregistrés, y compris les nouveaux arrivants et ceux qui sont revenus dans le camp après leur rapatriement.

Pour traiter les problèmes de protection des enfants à Dadaab, le HCR travaille avec Terre des Hommes et Save the Children International pour mettre en œuvre une intervention sur plusieurs fronts, en s'appuyant sur des structures communautaires telles que les volontaires de la protection de l'enfance, les personnes ressources issues de communauté, les services d'assistance adaptés aux enfants, les comités de protection de l'enfance, le conseil des parents d'accueil et des enfants confiés, les comités communautaires pour les adolescents, les comités pour le bien-être des enfants, les comités des centres d'éducation et du bien-être des enfants et les mères d'enfants afin de donner à la communauté les moyens de répondre aux préoccupations actuelles et émergentes en matière de protection de l'enfance. Ces structures communautaires suivent une formation qui les aide à identifier les problèmes de protection de l'enfance et à en étudier les causes et les conséquences. En outre, elles formulent des stratégies communes d'identification, de prévention et de réponse liées aux besoins et aux capacités de protection des enfants de chaque communauté. Ces interventions sont complétées par d'autres approches, notamment la sensibilisation, la médiation, la diffusion d'informations, la mise en place de voies de référence, les formations communautaires, la fourniture de services de conseil de base, la conception et la mise en œuvre de plans d'action communautaires par les adolescents, le traitement des cas à faible risque, le suivi des cas référés et la mise en relation de la communauté avec les prestataires de services pour renforcer et promouvoir la protection des enfants au niveau de la famille et de la communauté. Plus précisément :

- Terre des Hommes assure le placement temporaire et permanent d'enfants en famille d'accueil dans le cadre de son programme de placement familial.

- Un groupe de parents d'accueil de réfugiés travaille avec Save the Children International, en se portant volontaires pour prendre soin de mineurs abandonnés et non accompagnés (II y a 100 parents d'accueil à Ifo et Dagahaley et un total de 542 enfants placés en famille d'accueil).

- Les structures communautaires aident à identifier les enfants maltraités, à les signaler et à offrir des voies de référence et un soutien de suivi pour ces cas.

- Les structures communautaires telles que les comités de protection de l'enfance, les personnes ressources issues de la communauté et les volontaires de la protection des enfants participent aux processus de médiation des cas de maltraitance d'enfants à faible risque, ce qui permet de réduire le nombre de cas à faible risque au sein des communautés. Aux côtés des responsables communautaires, ces mêmes acteurs participent à l'identification et à la vérification des bénéficiaires des services de Terre des Hommes tels que le soutien aux moyens de subsistance, la formation et les services de gestion des cas.

- Les comités pour le bien-être des enfants contribuent à sensibiliser la communauté à la prévention de la maltraitance des enfants et aux pratiques culturelles néfastes, telles que le mariage des enfants, les mutilations génitales féminines et le travail des enfants. Ils soutiennent également Save the Children International dans l'amélioration de l'accès à la justice des enfants victimes d'abus. Ils utilisent des messages accessibles (adaptés aux enfants, etc.) dans leur diffusion d'informations aux enfants, aux parents/responsables d'enfants et aux membres de la communauté. En outre, ils sensibilisent les membres de la communauté aux effets immédiats et à long terme de la maltraitance des enfants, et à la manière d'identifier les enfants en détresse.

- Les membres de l'assemblée des enfants tiennent des sessions avec les partenaires au cours desquelles leurs préoccupations sont soulevées et les partenaires concernés y répondent. En général, les structures communautaires font office de mécanisme de compte rendu des réactions des bénéficiaires et des partenaires.

\section{Partenaire(s) :}

Save the Children International

Terre des Hommes

Pour de plus amples informations, contacter :

Charity Chomba : chomba@unhcr.org

Virginia Njoroge : njorogev@unhcr.org 


\section{Pratique émergente au Kenya : Cours sur les ondes de la radio communautaire}

$\int$ ans le camp de réfugiés de Dadaab, de nombreux élèves n'ont pas accès aux supports pédagogiques lorsque les établissements scolaires sont fermés. La diffusion de cours par radio permet d'atteindre une masse critique d'élèves, en particulier ceux qui peuvent apprendre de manière autonome et qui peuvent étudier avec une aide limitée en personne de la part des enseignants et des soignants. Les cours à la radio permettent aux enseignants de continuer à faire participer les élèves avec des supports d'apprentissage pertinents. Lorsqu'ils bénéficient d'applications interactives d'appoint telles que WhatsApp, les enseignants et les élèves ont la possibilité d'interagir plus étroitement, si la technologie requise est disponible. Les stations de radio communautaires sont essentielles à cette intervention, car de nombreuses radio FM qui diffusent des cours du programme d'enseignement national sont inaccessibles aux camps de réfugiés. Les stations de radio communautaires offrent donc aux étudiants réfugiés un accès égal aux supports pédagogiques fournis aux étudiants nationaux. Cette intervention actuelle implique une collaboration avec une station de radio communautaire (Radio Gargaar), qui a actuellement un rayon d'environ 40 km (représentant l'ensemble du camp et au-delà).

Lintervention implique l'utilisation d'un programme radio, qui est diffusé par SMS groupé et par l'intermédiaire des responsables communautaire une semaine avant la diffusion de lémission éducative. Les annonces radio sont également utilisées pour informer les réfugiés et la communauté d'accueil de la programmation radio prévue. Les étudiants doivent syntoniser le poste sur la fréquence de la radio selon l'horaire préétabli. Les familles qui partagent une clôture mitoyenne peuvent permettre à leurs voisin de profiter de l'appareil radio en le plaçant près de la clôture et en augmentant le volume. Les scripts radio pour chaque thème sont préparés par certains professeurs de matières et imprimés. Un enseignant qui a participé à la préparation des scripts est désigné pour répéter la leçon la veille de sa diffusion à la radio. Les groupes WhatsApp composés d'élèves et d'enseignants sont utilisés pour favoriser une interaction directe.

\section{Résultats}

II est prévu d'acquérir du contenu éducatif prêt à diffuser auprès de l'Institut kényan de rédaction des programmes d'études. Ce programme d'enseignement national remplacera les scripts radio actuels, dès qu'il sera disponible, tandis que les enseignants continueront à maintenir le contact avec les apprenants grâce aux groupes arborescents de WhatsApp.

\section{Partenaire(s) :}

Pour de plus amples informations, contacter:

Clara van Praag : vanpraag@unhcr.org

Alan Mwika : mwika@unhcr.org

\section{Étude de cas au Kenya : Engager les membres du club WASH de l'école}

'intervention du Conseil norvégien pour les réfugiés (CNR) dans le domaine de l'eau, de l'assainissement et de l'hygiène (WASH) implique l'inclusion des enfants et des jeunes et une approche à plusieurs facettes et à plusieurs niveaux. Dans les établissements scolaires, le CNR travaille en étroite collaboration avec les membres du club WASH (20 membres par établissement), en les formant aux approches de l'hygiène et de l'assainissement. À leur tour, les membres du club WASH formés s'adressent à leurs camarades de classe en tant que pairs éducateurs et partagent avec eux les informations et les approches qu'ils ont apprises. Le CNR forme également des adolescentes dans les établissements à la gestion de l'hygiène menstruelle. Au niveau communautaire, l'équipe WASH du CNR travaille avec les jeunes pour renforcer la participation des organisations communautaires et des groupes d'entraide aux activités WASH. Étant donné que l'approche du CNR est axée sur les enfants et les jeunes, son personnel est formé à la politique de protection de l'enfance, et les employés nationaux du CNR la signent au moment de leur embauche.

Partenaire(s) :

Conseil norvégien pour les réfugiés

Pour de plus amples informations, contacter :

Moe Miyahara : miyahara@unhcr.org

Oscar Nabiswa : nabiswa@unhcr.org

John Wagacha Burton : burtonj@unhcr.org 


\section{Pratique émergente en Ouganda : Projet de connectivité communautaire}

e projet de connectivité communautaire dans le quartier d'Imvepi est une initiative gérée par les jeunes qui répond au problème de l'oisiveté des jeunes dans le quartier, tout en répondant aux besoins de la communauté en matière de recharge téléphonique. Une source d'énergie stable et constante pour la recharge des téléphones est un enjeu majeur du camp-un défi qui entrave la communication et la coordination entre le HCR, ses partenaires et la communauté, y compris les conseils de protection des réfugiés. Le projet a débuté après l'installation de la cabine de recharge téléphonique communautaire alimentée par l'énergie solaire. L'intervention consiste à connecter les bâtiments communautaires (avec un accent particulier sur les bureaux de protection et les centres d'information et de soutien), à permettre l'accès au Wifi et à les transformer en centres connectés. Une fois cette connectivité effective, ces centres deviennent des lieux où les réfugiés peuvent accéder à l'information, communiquer avec le HCR et ses partenaires par l'intermédiaire de la ligne d'assistance téléphonique nouvellement créée (dans le cadre du mécanisme de référence et de résolution des problèmes), ou contacter à distance leurs proches en utilisant diverses applications de messagerie et d'appel vocal.

Au début du projet, les jeunes se sont activement mobilisés et ont participé à la sensibilisation de la communauté à l'activité de recharge téléphonique du centre, en s'adressant à tous les villages de la zone 1, y compris les lieux de culte, le marché, les lieux de distribution générale de nourriture et les événements sportifs. Dans le cadre de ce processus, les jeunes ont conclu des accords avec la communauté sur les tarifs de facturation de divers appareils : 300 shillings ougandais pour les téléphones ordinaires, 400 pour les smart phones, 500 pour les torches et 700 pour les ordinateurs portables. Les jeunes ont également fait participer les conseils à des séances de sensibilisation de la communauté afin d'obtenir un consensus solide autour le projet. Le président du conseil Il a notamment apporté un soutien très fort aux jeunes sur le projet dans le domaine du plaidoyer.

À ses débuts, le projet n'avait la capacité de charger que quelques appareils à la fois. Cependant, le HCR a récemment soutenu le centre en mettant 20 chargeurs de téléphone à sa disposition. II est actuellement possible de charger 12 à 15 téléphones par jour et d'autres appareils (que les ordinateurs portables, les torches et les banques d'alimentation).

La présence de la connectivité a renforcé la coexistence pacifique, en réunissant au centre des jeunes réfugiés et des jeunes de la communauté d'accueil pour prendre part ensemble à des jeux de société et interagir de bien d'autres manières. Grâce à cette plateforme d'interaction, les jeunes planifient une réunion avec leurs pairs de la communauté d'accueil au centre pour les intégrer dans l'équipe du projet de connectivité.

Les jeunes ont également lancé la vente de temps de communication pour téléphones portables grâce aux économies réalisées dans le cadre de l'initiative de recharge des téléphones. C'est un produit apprécié par nombre de personnes relevant de la compétence du HCR qui n'ont plus à parcourir de longues distances à cette fin. Les jeunes ont également plaidé en faveur de bloc de latrines à double fosse au centre. Cette infrastructure a finalement été fournie par le Comité international de secours avec le concours des jeunes qui y ont apporté leur contribution en creusant la fosse.

La responsabilité globale du projet incombe au HCR et au cabinet du Premier ministre, tandis que le partenaire de protection et les conseils sont responsables du suivi quotidien des résultats du projet.

\section{Partenaire(s) :}

Conseil danois pour les réfugiés

Cabinet du Premier ministre

Pour de plus amples informations, contacter :

William Ngeze : ngeze@unhcr.org

Faith Kamoyo : kamoyo@unhcr.org 


\section{Pratique émergente en Ouganda : Accès à l'enseignement secondaire}

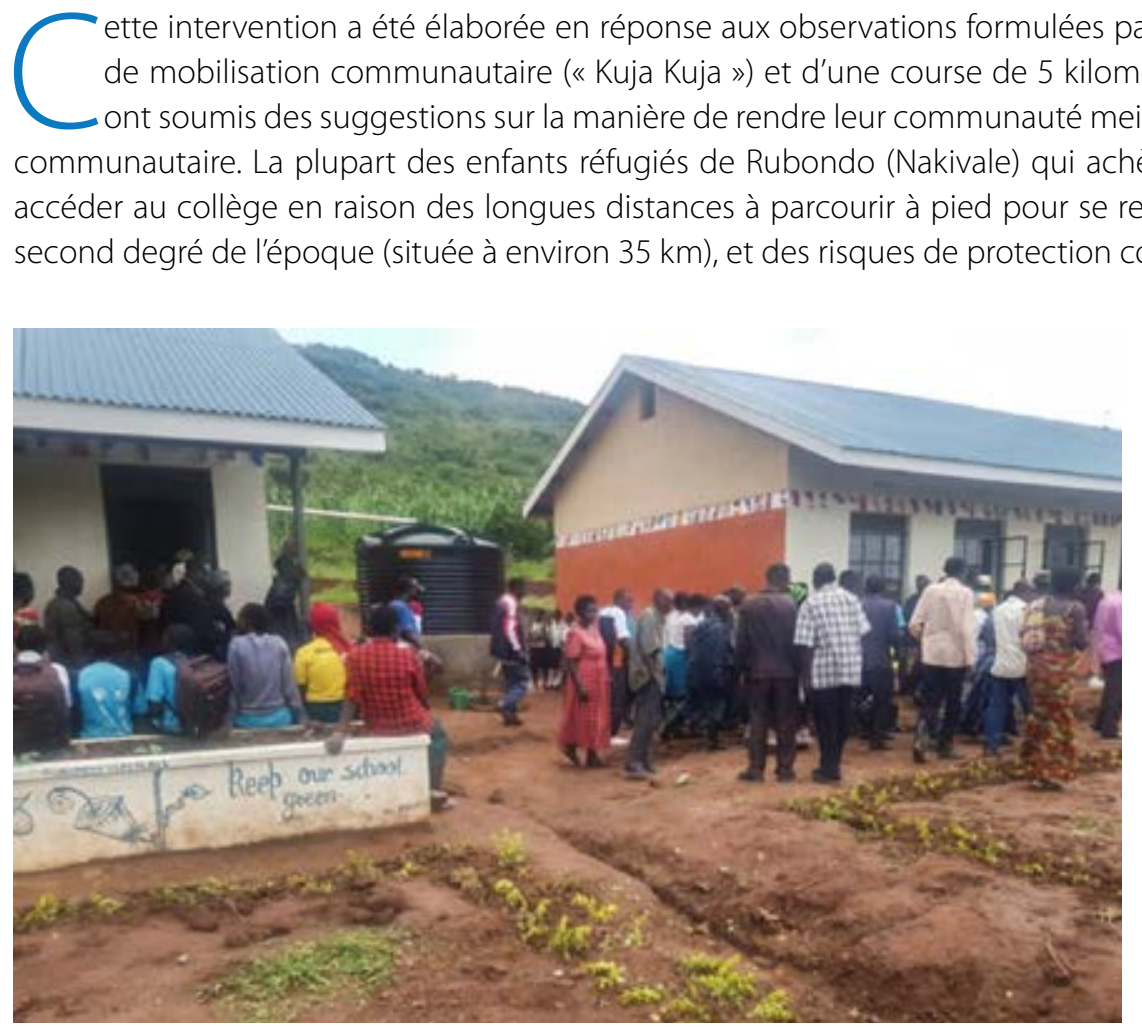

membres de la communauté, qui mobilisent tous les parents réfugiés et de la communauté d'accueil, en les encourageant à scolariser leurs enfants. Les membres de la communauté continuent également à prendre part à des activités mensuelles de participation communautaire, notamment le nettoyage de l'enceinte de l'école et la délimitation et la protection de ses limites contre les empiètements. Les parents contribuent aux frais de scolarité à hauteur de 100000 shillings ougandais (environ 27 dollars) par trimestre pour faciliter le fonctionnement de l'école, y compris le paiement des salaires des enseignants.

\section{Résultats}

- L'effectif est passé de 76 élèves (32 filles) à la fin de 2019 à 110 (52 filles) en mars 2020, avant la fermeture des classes en raison de la pandémie de COVID-19.

- Le taux de rétention était élevé ; 93 \% des élèves qui se sont inscrits en 2019 se sont réinscrits à la rentrée 2020.

- L'établissement dispose d'un programme de cantine scolaire dans le cadre duquel des repas sont servis aux élèves, ce qui facilite également la rétention.

- L'attitude positive de la communauté à l'égard de l'école est encourageant. La communauté soutient cette initiative qu'elle pilote elle-même, en aidant ses enfants à payer les frais de scolarité et à répondre à d'autres exigences telles que le matériel et les uniformes scolaires pour que leurs enfants puissent accéder à l'éducation dans l'établissement.

- La coordination et la collaboration avec les autres acteurs ont des bases solides. La direction de l'école travaille en étroite collaboration avec le gouvernement local du district, le HCR, le cabinet du Premier ministre, Windle International Uganda (le partenaire du HCR en matière d'éducation) et Alight, le partenaire qui soutient son développement.

- Développement des infrastructures : Avec le soutien des parties prenantes, notamment d'Alight, un laboratoire scientifique a été construit et achevé. II s'agit d'une exigence du Ministère de l'éducation et des sports pour toutes les écoles secondaires qui dispensent des cours de sciences.

\section{Partenaire(s) :}

Alight

Gouvernement local de district

Cabinet du Premier ministre

Windle International Uganda

\section{Pour de plus amples informations, contacter :}

Davis Mugumya : mugumya@unhcr.org

Sylvia Samanya : samanya@unhcr.org 


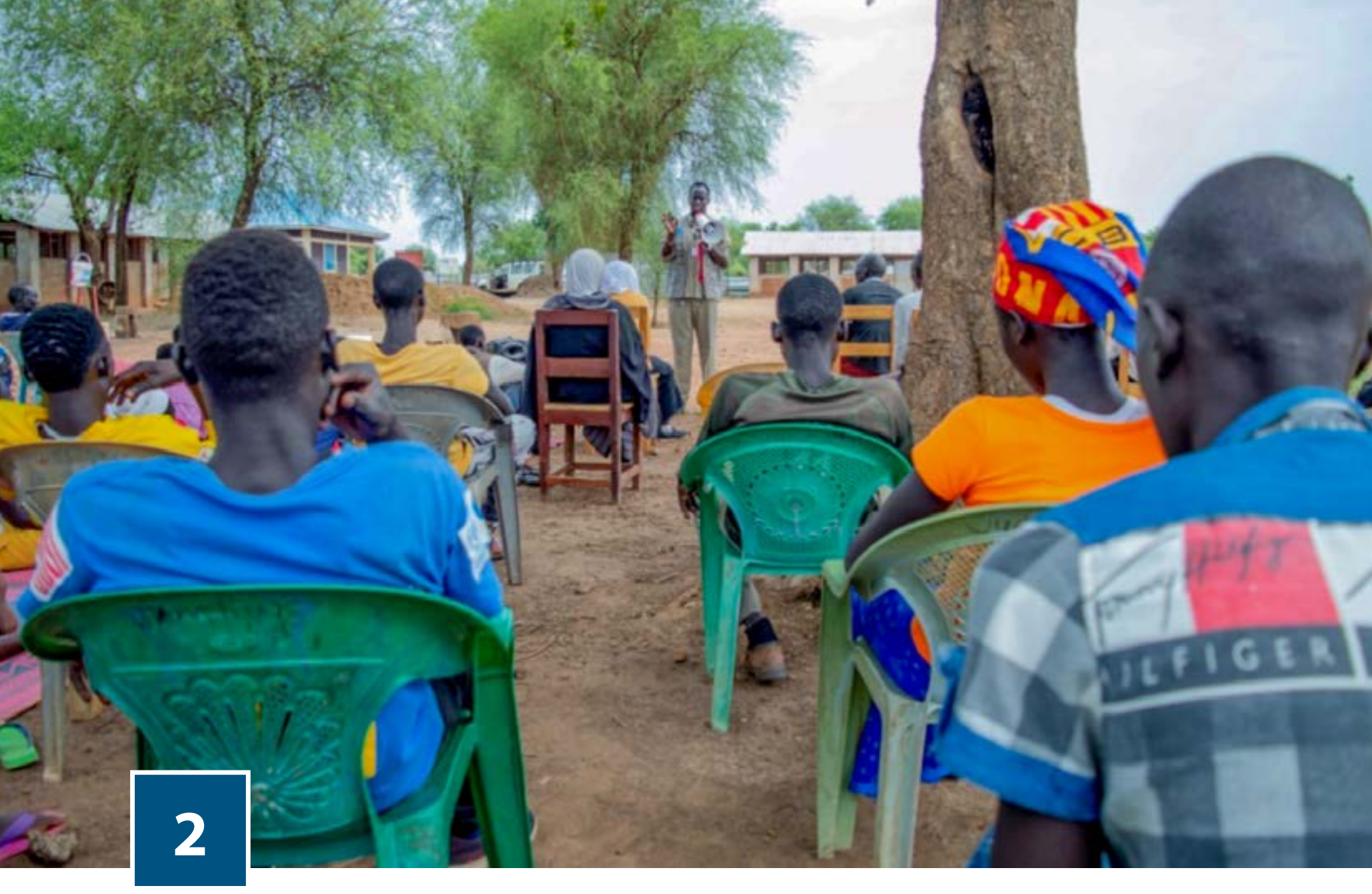

\section{PROTECTION COMMUNAUTAIRE}

\section{Pratique émergente à Djibouti : Contribution des communautés à la recherche de cas de tuberculose chez les réfugiés}

\footnotetext{
$\Gamma$

ette pratique vise à améliorer la détection des cas de tuberculose dans les camps de réfugiés et à promouvoir une initiation précoce au traitement par des activités de participation communautaire. L'autre but recherché est de renforcer le soutien aux clients atteints de tuberculose en leur fournissant des soins plus proches de leur domicile. L'intervention comprend six composantes principales, à savoir la formation de comités de santé, le renforcement des capacités des agents de santé communautaires et des travailleurs de la santé, des réunions-bilan trimestrielles, des réunions de plaidoyer, un soutien nutritionnel et un traitement à proximité de la communauté. Ces composantes sont présentées en détail ci-dessous.
}

- Mise en place d'un comité de santé de 10 membres dans chaque camp pour servir d'interface entre le système de santé et pour assurer la participation active des communautés.

- Formation de 17 agents de santé communautaire pour la mise en place d'un dépistage communautaire de la tuberculose dans les foyers, et formation de 15 agents de santé sur la tuberculose et la gestion de sa forme multirésistante.

- Bilan trimestriel des résultats de la recherche de cas dans la communauté par les agents de santé formés. Les résultats sont discutés avec l'ensemble de la communauté lors d'une réunion animée par les chefs de bloc/ section de la communauté, le représentant régional pour la lutte contre la tuberculose et le point focal du HCR. Les participants à la réunion s'accordent sur des recommandations pour améliorer la gestion de la tuberculose dans leurs communautés.

- Réunions de sensibilisation avec les responsables de communauté pour aider à lutter contre la stigmatisation de la tuberculose, du VIH et des patients.

- Un soutien nutritionnel est fourni par le HCR aux cas identifiés.

- Le programme national de traitement permet désormais aux tuberculeux de suivre un traitement au niveau du camp, ce qui leur permet de bénéficier du soutien de leurs communautés. 


\title{
Résultats
}

Avec le soutien des comités de santé communautaire et des responsables, les agents de santé communautaire ont jusqu'à présent référé un total de 140 cas suspects aux centres de santé pour diagnostic. Ce processus a permis d'identifier 12 cas réels de tuberculose au milieu de l'année, soit deux fois la proportion prévue (sur 6 cas) en milieu d'année.

\section{Partenaire(s) :}

Autorité intergouvernementale pour le développement

Ministère de la Santé de Djibouti

Pour de plus amples informations, contacter:

DrTongna Alain Rodrigue Zoure : zoure@unhcr.org

\section{Pratique émergente à Djibouti : Collecte communautaire participative des déchets solides par les personnes relevant de la compétence du HCR}

\begin{abstract}
uparavant assurés par une tierce partie, la collecte et l'enlèvement des déchets solides des ménages de réfugiés ont finalement été confiés à des personnes relevant de la compétence du HCR en raison de coupes budgétaires. Au cours des premières étapes, les campagnes de sensibilisation à la collecte des déchets n'ont enregistré qu'une faible participation de la communauté. Le partenaire d'exécution du HCR, la Direction de l'hydraulique rurale (DHR), a donc proposé d'adopter des stratégies de collecte similaires à celles utilisées dans les villes de Djibouti.

Le responsable de la communauté des réfugiés a formulé la stratégie de collecte des déchets ménagers en collaboration avec la DHR, en identifiant différents points de collecte des déchets et en prenant soin de n'oublier aucun ménage. Cette collaboration a conduit à l'établissement d'un calendrier hebdomadaire de collecte des déchets, d'un itinéraire de transit et de points de collecte, qui ont tous été communiqués aux membres de la communauté. Deux motocyclettes ont été achetées, pour parcourir quotidiennement l'itinéraire de ramassage, pendant que les réfugiés attendent aux différents points de collecte de leur zone avec les déchets ménagers à éliminer.

Pour assurer la qualité des services de collecte et de gestion des déchets ménagers, la DHR a recruté des réfugiés comme promoteurs de l'hygiène. Ces derniers suivent de sessions de renforcement des capacités pour assurer le maintien d'un cadre de vie sain dans leurs communautés respectives. Le maintien de l'hygiène que cette intervention favorise peut également servir à prévenir les conflits de voisinage entre la communauté d'accueil et les personnes relevant de la compétence du HCR. En outre, comme les responsables des communautés de réfugiés sont impliqués depuis l'élaboration de la stratégie jusqu'à sa mise en œuvre, et qu'ils supervisent également l'activité, ils sont libres de revoir et d'adapter l'intervention selon les besoins.
\end{abstract}

\section{Partenaire(s) :}

Direction de l'Hydraulique Rurale

Office national d'assistance aux réfugiés et sinistrés

Pour de plus amples informations, contacter: Moustapha Hassan Ahmed : mhaktulli@gmail.com Abdo Ali Kayad : abkalay@yahoo.fr

\section{Pratique émergente en Éthiopie : Organisation d'une élection des représentants des réfugiés en fonction de l'âge, du sexe et de la diversité (AGD)}

\footnotetext{
$\Lambda$ la suite des problèmes de sécurité découlant des affrontements ethniques qui ont coûté la vie à des réfugiés dans le camp de réfugiés de Kule, l'Administration pour les réfugiés et les rapatriés, l'agence de gestion du camp, a suspendu le Comité central des réfugiés (CCR) (qui était soupçonné d'avoir été compromis) et a temporairement établi un Comité de paix qui avait la double responsabilité de la résolution du conflit et de la direction de la communauté. Il était nécessaire d'établir un nouveau comité de représentants des réfugiés élus et digne de foi pour prendre la relève du Comité de paix et gérer la communauté du camp de réfugiés de Kule.
} 
Cette double intervention a consisté à élaborer des directives électorales qui ont permis une compréhension commune de toutes les parties prenantes et ont favorisé l'équité, la transparence et la responsabilité dans le processus électoral ; et à faciliter l'organisation de l'élection des représentants des réfugiés en s'inspirant de l'approche AGD.

Tandis que le HCR et l'ARRA ont facilité l'élaboration des directives électorales et l'organisation de l'élection, les membres du Comité de paix (membres intérimaires du CCR) ont piloté leur contribution au document et l'ensemble du processus électoral en informant la communauté au sens large sur les directives, en mobilisant ses membres pour l'élection et en supervisant le scrutin dans chaque sous-clan en qualité de commission électorale. La participation du Comité de paix (composé de tous les sous-clans), depuis l'élaboration des directives électorales jusqu'à l'élection proprement dite, visait à prévenir tout problème de protection résultant de la création d'un nouveau CCR. Les directives électorales contiennent des dispositions pour l'inclusion et protègent toutes les catégories de personnes, y compris les personnes ayant des besoins spécifiques.

\section{Résultats}

Grâce à cette pratique émergente, un nouveau comité central des réfugiés comprenant 15 membres élus a été mis en place avec succès. Pour la première fois, tous les clans et les personnes ayant des besoins spécifiques sont représentés dans la structure de direction. Le nombre de femmes au sein de cette structure de direction est également passé de 1 à 5 .

\section{Partenaire(s) :}

Administration pour les réfugiés et les rapatriés

Comité de paix des réfugiés

Pour de plus amples informations, contacter:

Dismas Nyamwana : nyamwana@unhcr.org

Mekdes Aschalew : aschalew@unhcr.org

\section{Pratique émergente en Éthiopie : Directives sur l'autonomisation des communautés}

es principes directeurs pour l'autonomisation des communautés ont été élaborés en collaboration par les dirigeants des communautés de réfugiés érythréens et le HCR, l'Administration pour les réfugiés et les rapatriés et d'autres

organisations non gouvernementales, dans le but de fournir un soutien financier et des biens qui permettraient aux réfugiés de subvenir à leurs besoins, et d'autonomiser la communauté en renforçant sa capacité à concevoir et à gérer des activités de protection dans les camps de réfugiés.

À cette fin, la Rehabilitation and Development Organization (RADO), un partenaire d'exécution du HCR travaillant avec les personnes handicapées et les personnes âgées, a bénéficié sur un budget de 770000 birrs (24 062,5 dollars) pour aider ses associations à créer des groupes d'entraide par le biais de projets d'autonomisation communautaire dans les quatre camps du Tigré : Adi Harush, Mai Aini, Hitstats et Shimelba. La RADO supervise huit associations (deux par camp) - quatre centrées sur les personnes handicapées et quatre centrées sur les personnes âgées). Les directives sur l'autonomisation des communautés ont fourni un cadre pour les projets d'autonomisation des communautés, et les activités pour ce faire ont inclus :

- Sensibiliser les bénéficiaires (en l'occurrence, les associations RADO) aux directives lors d'une réunion avec chaque association au cours de laquelle le HCR fournit des conseils si nécessaire. Des séances de questions et réponses ont été prévues pour s'assurer que les réfugiés comprennent bien les directives qui ont été traduites en Tigriana en vue d'une large diffusion.

- Rédaction de la proposition de projet de chaque association par les réfugiés en consultation avec les membres de leur communauté, en utilisant un formulaire d'autonomisation de la communauté. Les principaux éléments de la proposition comprenaient les besoins, les objectifs et les plans de gestion du projet.

- Fourniture d'un soutien supplémentaire à l'élaboration de propositions aux associations qui en ont besoin. Cela a permis de garantir la soumission de propositions dans les délais impartis et a également d'encadrer la planification du budget et des activités.

- Lidentification des sites/espaces de projets en coordination avec les responsables des camps.

- Des séances d'examen avec chaque association pour s'assurer de la qualité des propositions avant leur présentation à un comité de sélection.

- Formation de l'équipe de gestion par la RADO sur la gestion des petites entreprises avant le décaissement des liquidités et des actifs. 
- Suivi par une équipe conjointe (HCR, comité central des réfugiés, RADO) par le biais de visites périodiques sur le site du projet.

- Attribution d'une allocation mensuelle aux personnes qui gèrent directement le projet.

\section{Partenaire(s) :}

Administration pour les réfugiés et les rapatriés

Organisation de réhabilitation et de développement

Pour de plus amples informations, contacter:

Yamah Massaley: massale@unhcr.org

\section{Étude de cas au Kenya : Le sport au service de la protection et de la prévention de la violence}

e sport joue un rôle important dans la promotion de la protection des femmes, des hommes, des filles et des garçons dans différents secteurs tels que l'éducation, la santé et la coexistence pacifique dans le camp de réfugiés de Kakuma et la zone d'installation de Kalobeyei. Paradoxalement, le sport au Kenya (le football, en particulier) peut également déclencher des émotions fortes et des conflits entre des groupes qui partagent des identités ethniques et culturelles similaires.

Dans l'optique de réduire la violence et de promouvoir la coexistence pacifique entre les réfugiés et les communautés d'accueil, le HCR et ses partenaires ont établi un nouveau modèle de sport impliquant des équipes multinationales et multiethniques visant à favoriser un lien plus fort entre les différents groupes de personnes représentés par les réfugiés et les membres des communautés d'accueil.

Le nouveau modèle encourage le changement de comportement chez les filles, les garçons et les jeunes hommes et femmes, ainsi que les principes «ne pas nuire » par le biais d'un certain nombre d'activités clés:

- La création de ligues de football masculin («Kakuma Premier ») et féminin (« Diva League ») : Les ligues exigent que les équipes participantes soient multiethniques et multinationales.

- Formation professionnelle des arbitres : Le HCR et ses partenaires ont fourni des formations professionnelles aux arbitres, y compris aux membres des communautés d'accueil, car historiquement, la violence a été déclenchée par des décisions d'arbitres perçues comme erronées.

- Formation professionnelle des entraîneurs : En tant que dirigeants respectés d'équipes de football qui jouent un rôle important dans l'acceptation des décisions (même lorsqu'elles sont perçues comme erronées) et dans le calme des joueurs et des supporters qui peuvent ne pas comprendre les décisions des arbitres, les entraîneurs suivent également une formation dans le cadre de cette intervention.

- Gestion des terrains de sport et des équipes: Les conseils du sport sont des organes élus par les joueurs pour gérer les sports, comme le stipule la Kakuma Premier League. Des conseils et des secrétariats du sport ont été créés dans chaque zone à cet effet. Ils jouent un rôle important dans la planification des activités sportives à la satisfaction du public afin d'éviter les conflits potentiels. Les membres des conseils du sport sont formés aux compétences de leadership, à l'allocation des ressources et à la gestion.

- La création du « Kakuma United Football Club » : Pour renforcer la coexistence pacifique et promouvoir l'intérêt au-delà des enjeux locaux, le HCR et ses partenaires ont plaidé pour la création de ce club qui réunit les meilleurs joueurs des équipes de la Premier League pour qu'ils s'affrontent en deuxième division de la Kenya National Football League. Les joueurs du Kakuma United Football Club ont atteint le statut de célébrité locale, devenant de plus en plus connus des réfugiés et des Kényans.

- Programme de mentorat: Des formations au mentorat sont organisées, ciblant les filles ou les jeunes femmes, et les garçons à Kakuma. Grâce à des rencontres guidées avec des joueuses connues et respectées de la ligue (féminine) Kakuma Divas, les filles et les femmes sont sensibilisées et dotées de connaissances et de compétences sur diverses questions relatives à la protection des femmes. Des sessions de formation ont été proposées virtuellement pendant la période de la COVID-19, avec l'inscription de plusieurs acteurs.

- Complexe sportif de Kalobeyei : Le complexe comprend deux sites principaux, à savoir le stade et le centre polyvalent (salle). II vise à créer un environnement sûr et favorable aux réfugiés et à la communauté d'accueil. Les activités menées dans cet espace aideront également les jeunes à devenir des acteurs de protection actifs et des agents de changement positif, en assurant une plus grande participation des femmes, des jeunes filles, des réfugiés handicapés et d'autres groupes marginalisés. 
Partenaire(s) :

Fédération luthérienne mondiale

Pour de plus amples informations, contacter :

Amin Afridi : afridi@unhcr.org

Alexander Novikau : novikau@unhcr.org

Astone Ananda : ananda@unhcr.org

\section{Pratique émergente au Kenya : Arrangements en matière de soins de proximité pour les adultes non accompagnés (y compris les personnes âgées) vivant avec des formes graves de handicap et de maladie mentale}

\footnotetext{
$\square$ ette intervention vise à identifier et évaluer les adultes non accompagnés vivant avec des formes graves de handicap et de maladie mentale (y compris les personnes âgées) dans les camps de réfugiés ; fournir un dispositif de soins de proximité à ces adultes; veiller à ce qu'ils bénéficient d'interventions de protection et d'assistance appropriées ; et réduire la stigmatisation dont ils sont victimes. L'intervention a fait l'objet d'une phase pilote au profit de la population cible qui a séjourné longtemps dans les centres d'accueil en raison de ses besoins spécifiques. Auparavant, cette population ne pouvait pas être réinstallée dans la communauté car elle avait besoin d'un soutien communautaire supplémentaire.
}

Cette population cible est identifiée par la communauté, les partenaires ou le personnel du HCR, et est orientée vers une équipe de services spécialisés de la protection du HCR en utilisant la voie de référence interinstitutions établie par le camp de réfugiés de Kakuma. L'équipe des services spécialisés examine toutes les références et les affecte au partenaire concerné pour la gestion du dossier, en s'appuyant sur une approche multiorganisations appropriée. Le partenaire procède à une évaluation en consultation avec la personne relevant de la compétence du HCR, sa famille et les membres concernés de la communauté. Dans le cadre du plan d'action, le partenaire identifie une famille qui serait prête et capable de soutenir l'adulte vulnérable, ou un soutien communautaire plus large par le biais d'un système de surveillance mutuelle (généralement composé de voisins, d'amis, de membres du personnel de partenaire en charge de réfugiés, d'animateurs de jeunesse et de responsables communautaires qui sont prêts et capables de fournir un soutien). Dans le système de surveillance mutuelle, les fonctions de soutien sont identifiées et attribuées, y compris, par exemple, l'aide pour la cuisine, la blanchisserie ou la collecte des rations.

Le processus de placement d'un adulte vulnérable dans une famille d'accueil implique une série de consultations par le partenaire doublées des visites à domicile pour s'assurer que les modalités de soins et de placement sont bien comprises et acceptées par l'adulte vulnérable et la famille d'accueil. Le HCR et ses partenaires fournissent un soutien supplémentaire nécessaire, y compris la réinstallation, I'hébergement, les articles de secours essentiels, la relocalisation du lieu de l'assistance et la désignation d'arrangements de soins alternatifs. Après le placement, le partenaire effectue des visites de contrôle régulières, renforce les capacités de la communauté concernée et s'assure que l'adulte vulnérable et la famille sont informés sur la procédure pour entrer en contact avec le HCR et/ou le partenaire en cas de problème.

En résumé, les mesures prises au cours de cette intervention sont les suivantes:

- Les cas des groupes cibles sont identifiés.

- Les travailleurs des agences veillent à ce que la personne concernée soit enregistrée, puis orientée vers les agences compétentes pour une approche multifonctionnelle appropriée.

- Une évaluation tenant compte de la vulnérabilité est effectuée. La personne concernée consent à rencontrer les personnes identifiées considérées.

- Une présélection est effectuée, à travers notamment une rencontre avec la personne identifiée, la détermination de sa volonté de participer au programme, une visite à domicile et l'évaluation de l'accessibilité et de l'environnement général du domicile. Si la rencontre initiale n’a pas eu lieu à domicile, les modalités de placement sont expliquées.

- Le traitement a lieu ; il inclut la détermination de toute disposition logistique nécessaire à la réinstallation, par exemple, le logement, les articles non alimentaires, la collecte des rations, le bois de chauffage, etc.

- La relocalisation et le placement à domicile s'effectuent en organisant une réunion avec la personne concernée et l'aidant ainsi que sa famille, si elle est disponible, et en écoutant les préoccupations/questions éventuelles.

- Le suivi et la surveillance sont assurés, notamment en effectuant un contrôle à domicile bihebdomadaire pendant les deux premiers mois, en répondant à toute préoccupation éventuelle, en effectuant un point mensuel de la situation pendant trois mois supplémentaires, et par la suite, en clôturant le dossier, le cas échéant. 


\section{Résultats}

Au cours du premier mois de ce dispositif de soins de proximité, un total de 9 adultes non accompagnés vivant avec un handicap grave ou une maladie mentale, y compris des personnes âgées, ont été identifiés et mis en relation avec le soutien offert dans le cadre du programme.

\section{Partenaire(s) :}

African Inland Church

Humanity and Inclusion

Comité international de secours

Service jésuite des réfugiés

Pour de plus amples informations, contacter :

Leana Podeszfa : podeszfa@unhcr.org

Alexander Novikau : novikau@unhcr.org

Caroline Jeptoo : jeptoo@unhcr.org

Astone Ananda : ananda@unhcr.org

\section{Étude de cas au Kenya : Éviter la contamination de l'eau grâce aux ficelles fixées aux couvercles de jerricanes}

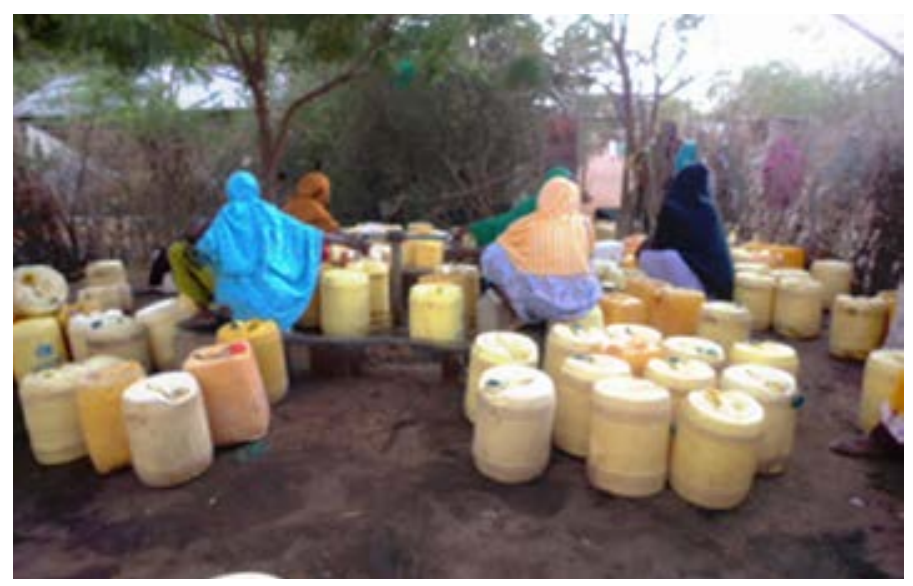

$\int$ es jerricanes de différentes capacités sont distribués deux fois par an dans le camp de réfugiés de Dadaab. II existe également un programme limité d'échange de jerricanes, qui permet d'échanger ceux qui sont abîmés, en fonction de la disponibilité du stock.

Les jerricanes sont fréquemment transportés des robinets aux ménages à l'aide d'une brouette, lorsque les conditions du sol le permettent. Bien que déconseillée par des messages de promotion de l'hygiène et des programmes de sensibilisation, la forme la plus courante de transport par jerricane consiste à le faire rouler sur le sol. Cette forme de transport nécessite que le couvercle du jerricane soit solidement fixé pour éviter les pertes d'eau et faciliter l'acheminement de l'eau. Pour y parvenir, les réfugiés de l'opération Dadaab ont mis au point un moyen de sécuriser les couvercles des jerricanes. Pour ce faire, on attache une ficelle au couvercle du jerricane, ce qui empêche l'eau de s'écouler et tient les germes nocifs à distance, malgré le dépôt de sable et de boue sur le jerricane lorsqu'on le fait rouler.

Partenaire(s) : s. o.

Pour de plus amples informations, contacter:

Osman Yussuf Ahmed : ahmedos@unhcr.org

\section{Étude de cas au Kenya : Comités de l'eau du marché}

G arantir un approvisionnement en eau potable accru ou maintenu dans les situations de réfugiés reste un objectif important. Des comités de l'eau du marché ont été créés dans les camps de réfugiés de Dadaab pour distinguer l'eau domestique de l'« eau commerciale » dans les communautés de réfugiés, et pour initier et instaurer une intervention qui permet de « payer pour accéder au service ». Les comités de l'eau du marché sont une coentreprise des réfugiés et du secteur des entreprises de la communauté d'accueil dans les trois camps. L'objectifà long terme de l'initiative 
est de faire passer la gestion des services d'eau pour les réfugiés à une société de distribution d'eau gérée par divers représentants des parties prenantes, à l'instar des approches des systèmes urbains de distribution d'eau au Kenya. Le HCR et CARE ont lancé un projet pilote de «kiosque de distribution d'eau » pour un comité de l'eau du marché. En cas de succès, le kiosque de distribution d'eau sera un précurseur de l'allocation mensuelle d'eau en vrac pour une famille d'environ 10 personnes. Une famille de cette taille se verrait attribuer 20 litres d'eau par jour et par habitant, soit 6000 litres par mois. La quantité d'eau allouée sera puisée à tout moment de la journée au kiosque de distribution d'eau. Toute utilisation d'eau excédant ce volume doit être payée.

Partenaire(s) :

CARE

Pour de plus amples informations, contacter:

\section{Pratique émergente au Kenya : « Le lien spirituel » - Les églises urbaines dirigées par des réfugiés comme ressources}

es communautés de personnes relevant de la compétence du HCR dans les zones urbaines du Kenya ont besoin de mécanismes d'adaptation pour leur permettre de survivre aux défis de la vie en ville dans un pays étranger. La foi peut contribuer de manière significative à promouvoir la protection sociale et la résolution des conflits tout en aidant à maintenir un sentiment d'identité chez les réfugiés.

À Nairobi, certains réfugiés ont créé leurs propres lieux de culte. Ces lieux de culte sont principalement conçus pour répondre aux besoins spirituels des réfugiés, tout en reproduisant l'expérience de l'église du pays d'origine des réfugiés. L'une de ces églises, située dans la banlieue de Nairobi (au quartier Kitengela), est dirigée par un pasteur réfugié, qui se trouve être également un leader de la communauté des réfugiés. L'église rassemble une congrégation de réfugiés qui ont accès à toute une série de services, notamment l'hébergement temporaire des nouveaux arrivants dans la zone, l'intégration des nouveaux arrivants auprès des autres réfugiés et au sein de réseaux, le soutien alimentaire et matériel (malgré des ressources limitées) et des cours de langue de base, qui améliorent la communication et les moyens de subsistance.

L'église est également membre de la «Kitengela Pastors Fellowship », un forum qui offre des possibilités de mise en réseau avec les églises des communautés d'accueil, et grâce auquel certains des conflits entre les réfugiés et leurs hôtes ont été résolus.

\section{Résultats}

Les églises de réfugiés procurent à leurs fidèles un sentiment d'appartenance, ce qui est important pour le bien-être psychosocial. Le lien entre les églises de réfugiés et celles des communautés d'accueil ouvre des possibilités de dialogue et de soutien aux personnes relevant de la compétence du HCR. II apporte également des solutions pratiques à certains problèmes que rencontrent les réfugiés dans leurs relations avec l'administration et la police locales. Linteraction entre l'église des réfugiés et celles de la communauté d'accueil a également amélioré l'accès des réfugiés aux informations et aux contacts utiles pour obtenir un soutien pendant la pandémie.

\section{Partenaire(s) :}

Groupe de travail sur la protection communautaire

Secrétariat des affaires des réfugiés

\section{Pour de plus amples informations, contacter :}

Jackson Karugu : karugu@unhcr.org 


\section{Étude de cas au Kenya : Comités WASH communautaires}

e camp de réfugiés de Kakuma et les colonies de Kalobeyei sont caractérisés par un terrain semi-aride, des conditions de chaleur extrême, des inondations fluviales et une pénurie générale d'eau. Pour favoriser l'appropriation des questions liées à l'eau, à l'assainissement et à l'hygiène par les communautés, chaque quartier de Kakuma met en place un comité WASH.

Les comités WASH sont des groupes de bénévoles composés de quatre à six personnes chargées de superviser toute question qui se pose dans le secteur de l'eau, de l'assainissement et de l'hygiène. À côté des deux chefs de bloc qui sont automatiquement membres du comité WASH de bloc, les autres membres sont désignés à l'issue de concertations avec les membres de la communauté. Habituellement, les membres sélectionnés représentent différentes couches de la communauté, telles que les femmes, les jeunes, les minorités, les personnes ayant des besoins spécifiques et les personnes vivant avec un handicap.

À Kakuma et Kalobeyei, l'approvisionnement en eau se fait à partir de 19 forages reliés à un réseau de citernes en acier surélevées et un réseau de distribution avec des robinets dans la communauté. Les forages sont alimentés en grande partie par des groupes électrogènes, mais aussi par des panneaux solaires, lorsqu'ils sont disponibles.

La concentration des usagers et les longs temps d'attente aux points d'eau entraînent souvent un stress et une frustration chez les personnes qui vont chercher l'eau. Les conflits aux points d'eau peuvent être source de tensions et de désengagement de la communauté. Pour atténuer ce problème, les comités WASH élaborent des calendriers de distribution par le biais d'un dialogue avec la communauté. Chaque famille est ensuite programmée pour l'un des trois tours de distribution d'eau. L'eau est distribuée trois fois par jour (une fois le matin, une fois à midi et une fois le soir avant la tombée de la nuit). La distribution d'eau avant la tombée de la nuit permet d'assurer la sécurité des femmes et des filles, à qui la tâche d'aller chercher de l'eau incombe souvent.

Certains membres de la communauté sont formés à la réparation des installations WASH, y compris les robinets, qui connaissent souvent des pannes. L'implication de la communauté dans l'engagement et la résolution de ses propres problèmes liés à l'eau, à l'assainissement et à l'hygiène, favorise l'autonomisation et le renforcement de ses capacités.

LeConseil norvégien pour les réfugiés affecte du personnel national de protection pour soutenir les comités WASH et s'assurer que les projets dans ce domaine sont mis en œuvre conformément aux normes, principes et directives de protection, en mettant l'accent sur la résolution des conflits et la sauvegarde des programmes. Le secteur WASH du Conseil norvégien pour les réfugiés travaille en étroite collaboration avec les départements du HCR (protection, unité communautaire, unité de terrain, santé, éducation) pour traiter toute question de protection liée à l'eau, à l'assainissement et à l'hygiène. Une attention particulière est accordée aux ménages dirigés par des femmes, aux enfants non accompagnés, aux personnes âgées et aux personnes ayant des besoins spécifiques en vue de leur inclusion. Le Conseil norvégien pour les réfugiés implique la communauté dans toutes les phases de la gestion du cycle de projet WASH. Son équipe WASH participe à une réunion mensuelle des responsables et des partenaires de la communauté afin de fournir un retour d'information et de partager des informations avec la communauté. Le Conseil norvégien pour les réfugiés dispose également d'un mécanisme de plainte, de réponse et de retour d'information qui encourage le dialogue communautaire.

\section{Partenaire(s) :}

Conseil norvégien pour les réfugiés

Pour de plus amples informations, contacter:

Oscar Nabiswa : nabiswa@unhcr.org

Moe Miyahara : miyahara@unhcr.org 


\section{Étude de cas au Rwanda : Gouverneurs de village}

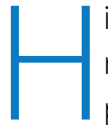
istoriquement, dans le camp de Mahama, les rapports du HCR n'étaient que très peu informés par les récits des réfugiés, s'appuyant principalement sur les informations des partenaires et sur un suivi occasionnel avec un personnel limité. La nécessité d'impliquer plus délibérément les réfugiés est apparue dès le début de l'urgence dans le camp, lorsquil a été découvert que les réfugiés au niveau de la base manquaient d'informations sur la manière de résoudre leurs propres problèmes et sur les endroits où ils pouvaient accéder aux services du camp de Mahama. La direction du HCR a conçu l'intervention des « gouverneurs de village » pour garantir que les personnes relevant de la compétence du HCR aient accès à des informations précises en temps opportun ; renforcer la capacité des réfugiés à résoudre de manière indépendante des problèmes mineurs au sein de la communauté sans intervention des partenaires d'exécution ; et surveiller la prestation de services par les partenaires d'exécution par le biais de comptes communautaires, en tenant les partenaires responsables en cas de divergences.

L'initiative des gouverneurs de village consiste à affecter un membre du personnel du HCR à la supervision d'un village du camp de réfugiés de Mahama, qui accueille environ 250 à 480 familles. Ces agents sont chargés de veiller à ce que les réfugiés résidant dans leurs villages soient correctement informés des services fournis dans le camp, bénéficient d'un éventail de services disponibles et soient en mesure de résoudre les problèmes mineurs de la communauté (ou de mettre en place des mécanismes à cette fin).

Surnommés " gouverneurs », ces employés du HCR tiennent diverses réunions avec les chefs de village, collaborent avec eux pour organiser des rassemblements de masse une fois par mois ou tous les trimestres. Ces sessions communautaires sont utilisées pour diffuser des informations sur les services disponibles, les règles du camp, la sécurité et la sûreté, les solutions durables, la protection des enfants et la violence sexuelle et fondée sur le genre, ainsi que des messages de mobilisation communautaire pour trouver des solutions aux problèmes communautaires de manière indépendante. Des séances de questions et réponses font partie intégrante de ces réunions.

Les gouverneurs invitent parfois des fonctionnaires et différents partenaires d'exécution à transmettre des messages importants ou à fournir des informations sur les défis soulevés par la communauté. Les gouverneurs participent également à des travaux communautaires aux côtés des habitants de leurs villages avant les réunions, afin d'encourager le travail communautaire et la solidarité. De temps en temps, les gouverneurs organisent des réunions spéciales dans les cas où de nouvelles formes d'assistance sont disponibles ou lorsqu'un message urgent doit être transmis aux résidents (par exemple, le déploiement d'interventions impliquant des numéraires, la distribution de gaz, les informations relatives aux exercices de vérification).

Les rapports de ces réunions sont diffusés auprès de l'équipe de direction du HCR, ce qui permet d'identifier des modèles similaires, de tirer des enseignements essentiels et de prévoir des interventions fondées sur les besoins.

\section{Partenaire(s) : s. o.}

Pour de plus amples informations, contacter:

Emilia Lobti : lobti@unhcr.org; rwaki@unhcr.org 


\section{Pratique émergente au Rwanda : Cliniques mobiles de protection}

es réunions hebdomadaires de coordination entre le HCR et les partenaires d'exécution dans le camp de réfugiés de Mahama (aux fins de coordonner toutes les activités de protection) ont révélé la nécessité de passer plus de temps dans la communauté des réfugiés, en s'assurant que les personnes relevant de la compétence du HCR ont accès aux bons messages, comprennent la voie de référence pour les cas de protection des enfants et de violence sexuelle et fondée sur le genre, et ont la possibilité de poser des questions liées à tout problème de protection dans le camp. Il a été décidé que la meilleure stratégie pour fournir un front solide et résoudre les problèmes de manière simultanée était de travailler conjointement à cette fin. Un programme de roulement a été élaboré à cet effet par le HCR pour guider le travail des partenaires dans la coordination et l'organisation de ces visites communautaires conjointes en étroite collaboration avec les chefs de village au cours des trois premières semaines de chaque mois. La dernière semaine du mois est réservée à la planification de la réunion de coordination de la protection, et à la participation des partenaires et des dirigeants de la communauté.

Grâce à ces efforts, chaque jeudi, une grande réunion de village est organisée dans la communauté de manière tournante entre les 18 villages du camp Mahama, en plus des établissements scolaires, des marchés et de la communauté d'accueil. Les réunions sont organisées conjointement par le HCR, le Ministère en charge de la gestion des urgences (MINEMA) et les partenaires chargés de la protection, en collaboration avec les chefs de village et de communauté, les affaires sociales et les structures communautaires (groupes antiviolence sexuelle et fondée sur le genre, groupes de jeunes, activistes communautaires, troupes de théâtre, groupes de danse). Cette pratique est appelée « clinique mobile de protection ». La clinique mobile de protection commence généralement par une présentation du chef du village/de la communauté, suivie d'une danse, d'une chanson, d'un poème ou d'un sketch sur une question de protection donnée dans la communauté. Les partenaires font ensuite des présentations sur les services disponibles et sur les réponses et les mesures de prévention de la criminalité, de la protection des enfants et des problèmes liés à la violence sexuelle et fondée sur le genre.

Les cliniques mobiles de protection offrent une plateforme au HCR, au MINEMA et à leurs partenaires pour sensibiliser la communauté à divers sujets de protection et diffuser les informations et les annonces les plus récentes. Une session est intégrée à l'intervention pour les questions, les plaintes et les réactions de la communauté ainsi que les réponses des partenaires. Les cas individuels et sensibles ne sont jamais abordés lors des visites de la clinique. Au lieu de cela, les partenaires fournissent des informations générales sur les endroits où l'on peut joindre les différentes institutions en cas de besoin, et les cas identifiés qui émergent au cours des sessions sont référés aux partenaires concernés. La communauté se voit rappeler en permanence ses droits, ses obligations et le respect des règles et règlements du camp.

La clinique mobile est gérée d'une manière attrayante et agréable, avec des responsables de la communauté partenaires qui emploient un langage respectueux et responsabilisant. Un langage adapté aux enfants est utilisé lorsqu'on s'adresse à eux, et la participation des femmes, des hommes, des filles et des garçons de tous les milieux est encouragée par la mobilisation.

\section{Résultats}

Les cliniques mobiles de protection sont un moyen d'atteindre la communauté de manière dynamique, plutôt que d'attendre que ses membres recherchent les partenaires. Grâce à cette pratique, les partenaires sont en mesure de rester au fait des réalités auxquelles les communautés de réfugiés sont confrontées, et d'enregistrer diverses plaintes et réactions, ainsi que la possibilité d'y répondre.

\section{Partenaire(s) :}

Ministère en charge de la gestion des urgences

Save the Children International

Alight

Agence adventiste du développement et de l'aide humanitaire

Comité international de la Croix-Rouge

Legal Aid Forum

Fraternité des prisons au Rwanda

Pour de plus amples informations, contacter :

Emilia Lobti : lobti@unhcr.org 


\title{
Pratique prometteuse au Rwanda : Associations d'épargne et de prêt volontaires
}

\begin{abstract}
$\Lambda$ la suite de consultations avec les chefs de communauté, les réfugiés handicapés et leurs familles dans les camps de réfugiés burundais, les défis auxquels sont confrontés les personnes handicapés sont devenus évidents. Parmi ceux qui ont été mis en lumière, on peut citer, notamment l'accès limité aux services de subsistance disponibles, l'exposition au risque d'abus et d'exploitation et la stigmatisation et la discrimination. Ces conclusions, qui s'alignent sur l'intégration de l'âge, du sexe et de la diversité du HCR, ont relevé la discrimination à laquelle sont confrontés les réfugiés en situation de handicap, ainsi que des problèmes récurrents de protection/sécurité et des lacunes dans la fourniture de services. Les associations d'épargne et de prêt volontaires qui mettent l'accent sur l'intégration des réfugiés en situation de handicap sont nées de ces réalités, dans le but de promouvoir l'autonomie de cette population et son inclusion dans les programmes.
\end{abstract}

En collaboration avec des partenaires de protection communautaires, le HCR a organisé de multiples activités visant à renforcer l'estime de soi et la confiance en soi des personnes handicapées, à travers notamment la sensibilisation de la population du camp de Mahama et de la communauté d'accueil aux sept principes généraux de la Convention relative aux droits des personnes handicapées, à savoir :

- le respect de la dignité intrinsèque

- l'autonomie individuelle, y compris la liberté de faire ses propres choix, et l'indépendance des personnes ; le principe de la non-discrimination

- la participation et l'intégration pleines et effectives à la société

- le respect de la différence et l'acceptation des personnes handicapées comme faisant partie de la diversité humaine et de l'humanité

- l'égalité des chances

- l'accessibilité

- l'égalité entre des femmes et des hommes

Ces séances ont permis de sensibiliser les personnes handicapées à leur droit et à leur liberté de participer pleinement à la société. Elles ont donc formé un comité de handicap qui les a aidées à lancer leurs propres activités génératrices de revenus, notamment des associations d'épargne et de prêt volontaires, afin de réduire leur dépendance à l'aide et d'accroitre leur participation socioéconomique.

Pour permettre la durabilité des associations créées, le HCR, en collaboration avec Humanité et Inclusion, a lancé des sessions de renforcement des capacités des associations sur les thèmes tels que la direction de l'association, la participation et le rôle de chacun de ses membres, les moyens d'établir les règles et les règlements des associations, la règle de la fixation des objectifs, le suivi et évaluation au sein de l'association, la documentation, les compétences en matière d'épargne, la gestion de l'épargne et le développement des prêts, la tenue de livres et le travail avec les institutions financières. Les associations ont été encouragées à ouvrir des comptes dans des institutions de microfinance afin d'améliorer leurs chances d'accéder à des prêts bancaires pour des solutions économiques durables.

Le HCR et ses partenaires d'exécution organisent des sessions de formation et tiennent des réunions régulières avec les associations, en veillant à ce qu'elles respectent le principe « ne pas nuire », en donnant aux membres fondateurs les moyens d'agir de manière non discriminatoire. Le HCR surveille également le respect par les associations d'épargne et de prêt volontaires des règlements convenus, intègre une composante d'épargne dans leurs initiatives et s'efforce de consolider leur autonomie, leur indépendance et leur dignité. Des réunions régulières entre les partenaires de mise en œuvre et les bénéficiaires ainsi qu'un soutien en matière de comptabilité, en plus d'autres stratégies, garantissent que les associations ont mis en place un système de suivi et de responsabilité en matière de transparence.

\section{Résultats}

- Le camp de réfugiés de Mahama compte actuellement 15 associations formées principalement par des personnes handicapées, bien que la participation soit ouverte à tous les membres de la communauté.

- Les membres de la communauté, y compris les dirigeants, ont adhéré à des associations génératrices de revenus initiées par des personnes handicapées. Dans le camp de réfugiés de Mahama, toutes les associations d'épargne et de prêt volontaires combinées sont composés de 361 membres de la communauté (159 hommes, 202 femmes). Parmi eux, 90 sont des personnes handicapées.

- Au total, 15 associations ont ouvert des comptes bancaires.

- Les associations sont maintenant sollicitées par diverses parties prenantes (par exemple, le gouvernement 
rwandais et les ambassades étrangères) pour participer à des compétitions, des expositions et des cérémonies de remise de prix dans les camps, ainsi qu'à d'autres occasions spéciales. Lors des réunions de masse et des commémoration diverses journées internationales, des créneaux horaires sont alloués aux réfugiés handicapés, à leurs familles et à leurs partenaires pour partager des témoignages sur les effets des associations qui ont changé leurvie.

- Les communautés témoignent que les réfugiés handicapés ont également la capacité et la force de soutenir leur famille et la communauté dans son ensemble.

Partenaire(s) :

Humanité et Inclusion

Pour de plus amples informations, contacter :

Emilia Lobti : lobti@unhcr.org; rwaki@unhcr.org

\section{Pratique émergente en Ouganda : Renforcer la voix des réfugiés grâce à un forum d'engagement des réfugiés}

\footnotetext{
- n tant que plus grand pays d'accueil de réfugiés en Afrique et l'un des premiers à mettre en œuvre le Cadre d'action - global pour les réfugiés, l'Ouganda a adopté sa feuille de route en la matière en janvier 2018. Elle définit la vision pour renforcer la mise en œuvre d'une approche globale dans sa réponse afin d'alléger la pression sur les districts d'accueil et de répondre aux besoins des réfugiés et des communautés d'accueil. La feuille de route du Cadre d'action global pour les réfugiés en Ouganda identifie la satisfaction des besoins des réfugiés et des communautés d'accueil comme l'une de ses composantes essentielles dans le pays.
}

Le groupe de pilotage du Cadre d'action global pour les réfugiés est un organe décisionnel de haut niveau qui se réunit tous les trimestres pour orienter stratégiquement la mise en œuvre du Cadre en Ouganda. Le groupe de pilotage est une plateforme unique, qui rassemble des ministères, des départements et des organismes publics, des partenaires bilatéraux et multilatéraux internationaux, des ONG internationales et locales, le secteur privé, les réfugiés et les communautés d'accueil. Deux sièges du groupe de pilotage du Cadre d'action global pour les réfugiés sont réservés aux réfugiés pour représenter 1,44 million de réfugiés dans le pays.

Le Forum d'engagement des réfugiés a été créé en octobre 2018 pour s'assurer que les communautés touchées sont désormais représentées de manière efficace par leurs dirigeants au sein du groupe de pilotage du Forum. Avant la création du Forum, les structures de direction des réfugiés étaient limitées au niveau de l'établissement (Conseils de protection des réfugiés) sans engagement structurel au niveau national. En raison de cette limitation, l'engagement des parties prenantes avec la communauté des réfugiés au niveau national était ad hoc et non systématique. Actuellement, le Forum est composé de 37 membres issus de la direction de la zone d'installation, tenant compte de la diversité de la démographie, du sexe et des groupes d'âge. II s'agit de deux dirigeants élus de chaque zone d'installation, des représentants des jeunes et des dirigeants communautaires de Kampala. Les membres du Forum élisent deux membres du groupe - une femme et un homme de différentes nationalités et de différentes localités - pour représenter les réfugiés lors des réunions trimestrielles du groupe de pilotage pour une durée d'un an. Les réunions du Forum ont aussi lieu tous les trimestres, avant chaque réunion du groupe de pilotage du Cadre d'action global pour les réfugiés, afin d'informer le groupe de toutes les décisions qui concernent les réfugiés. Deux représentants élus du Forum ont assisté à toutes les réunions du groupe de pilotage du Cadre d'action global pour les réfugiés depuis octobre 2018. Par le biais du Forum, la communauté des réfugiés est également tenue informée des discussions et des décisions du groupe de pilotage du Cadre d'action global pour les réfugiés.

Le groupe de travail du Cadre d'action global pour les réfugiés organise et facilite les réunions du Forum. Le groupe de travail est composé du cabinet du Premier ministre, du Secrétariat du Cadre d'action global pour les réfugiés, du HCR et des ONG internationales (CARE, Oxfam, IRC et autres). Les bureaux régionaux des réfugiés et les commandants de zone d'installation du cabinet du Premier ministre travaillent en étroite collaboration avec les sous-bureaux ou des bureaux hors siège du HCR pour soutenir le travail des membres du Forum en faisant remonter à la communauté les questions abordées lors de ses réunions et en consultant la communauté sur les problèmes qu'elle doit signaler au Forum et au groupe de pilotage du Cadre d'action global pour les réfugiés. 
Le Forum d'engagement des réfugiés permet une participation significative de la communauté des réfugiés dans la planification, la mise en œuvre et le suivi du Cadre d'action global pour les réfugiés en Ouganda. La structure de direction des réfugiés est maintenant en place du point de vue structurelle au niveau du village - zone - colonie - national (colonies transfrontalières) pour partager les discussions sur le terrain au forum national et vice-versa. Le Forum est un mécanisme de responsabilisation important pour toutes les parties prenantes dans la réponse aux réfugiés et il contribue également à renforcer l'autonomie des réfugiés, en répondant efficacement aux besoins des réfugiés et des communautés d'accueil.

Deux représentants des réfugiés du Forum ont participé au Forum mondial sur les réfugiés, à l'Autorité intergouvernementale pour le développement, à la Conférence internationale de Tokyo sur le développement de l'Afrique et à d'autres engagements internationaux pour partager leur expérience et leurs connaissances et pour discuter des questions qui ont un impact sur leur communauté.

Partenaire(s) : divers

Pour de plus amples informations, contacter :

Yoko Iwasa : iwasa@unhcr.org

Leslie Velez: velez@unhcr.org

Ndahiriwe Innocent : indahiriwe7@gmail.com

Rick Bartoldus : Rick.Bartoldus@rescue.org

Annet Kyambadde : Annet.Kyambadde@care.org

\section{Pratique émergente en Ouganda : Création de bureaux communautaires du conseil d'aide aux réfugiés, formation de groupes de soutien aux personnes ayant des besoins spécifiques et initiative de développement des compétences}

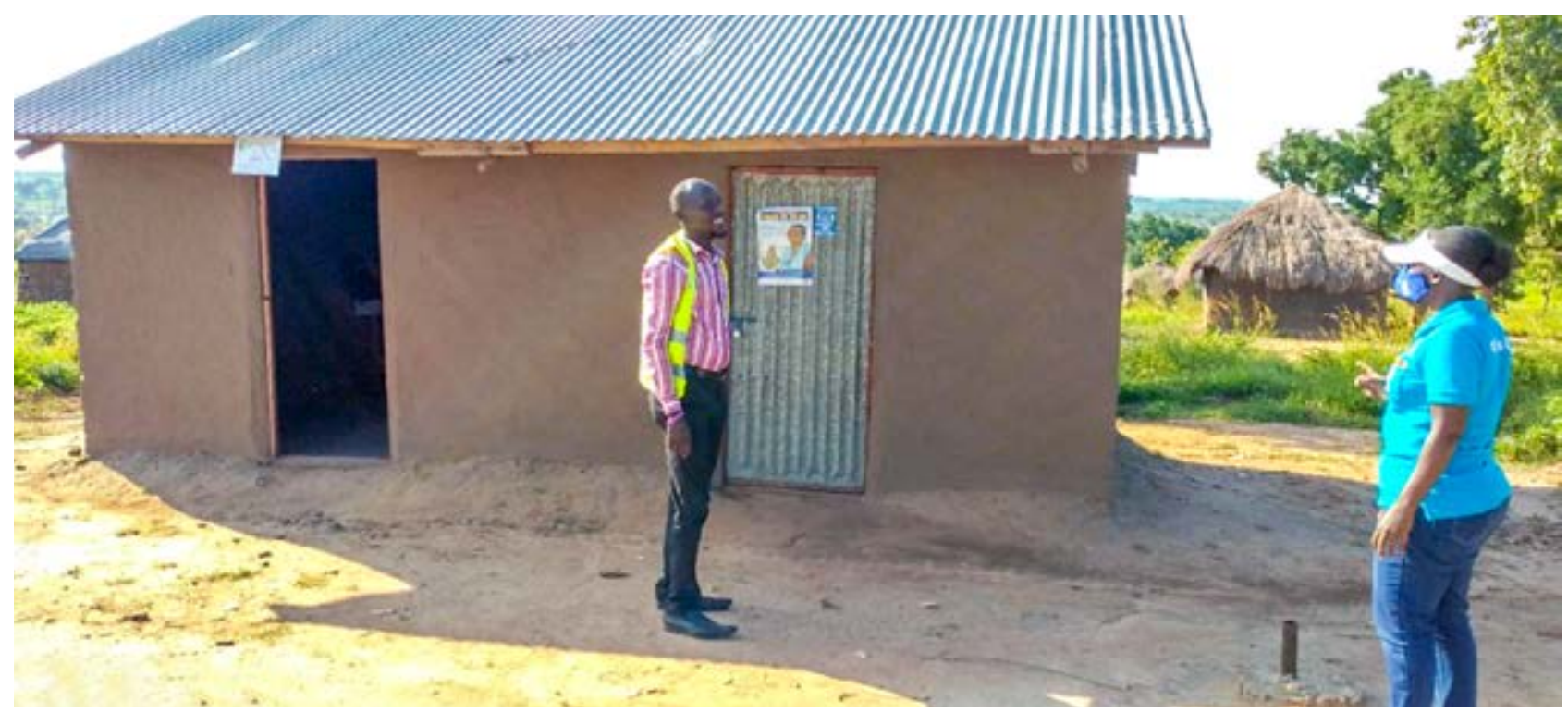

a construction d'espaces semi-permanents du conseil d'aide aux réfugiés dans la zone d'Ofua était une initiative purement communautaire menée par les dirigeants du conseil et visant à améliorer leur capacité à coordonner plus efficacement avec les partenaires et la communauté tout entière. Les espaces de bureaux des conseils d'aide aux réfugiés ont été considérés comme essentiels pour créer un environnement propice et confidentiel pour l'engagement entre les membres de la communauté, les partenaires et les conseils, tout en renforçant la participation de la communauté, en maintenant la transparence et la responsabilité et en observant les voies de référence existantes.

Des bureaux de conseil d'aide aux réfugiés ont été installés dans les 6 villages de la zone d'Ofua. Ces espaces permettent aux réfugiés et aux partenaires d'approcher plus facilement les dirigeants des conseils d'aide aux réfugiés pour obtenir leur soutien et leur participation à la planification, à la mise en œuvre et au suivi des prestations de services. Les espaces sont ouverts à plein temps et au moins un membre exécutif du conseil d'aide aux réfugiés est présent pour s'occuper des 
questions, y compris les plaintes ou les préoccupations individuelles, le partage des réactions et le besoin d'un espace de réunion.

En outre, les personnes ayant des besoins spécifiques de la zone d'Ofua ont créé six groupes de soutien fonctionnel (un par village), et un septième groupe supplémentaire (composé de 19 membres et représentant la zone) a été désigné pour superviser les six groupes basés dans les villages. Les groupes de soutien des personnes ayant des besoins spécifiques se réunissent régulièrement pour discuter des questions qui les concernent et pour élaborer des solutions locales à ces problèmes. Chaque groupe est composé d'un président, d'un vice-président, d'un trésorier et d'un secrétaire qui tient un registre des défis et réalisations du groupe. Les groupes de soutien sont utilisés comme un mécanisme pour : aider à sensibiliser les personnes ayant des besoins spécifiques à leurs droits et obligations, leur fournir des conseils de base en cas de besoin, et les engager dans des activités de subsistance (par exemple, l'élevage de volaille, le jardinage), et dans le commerce de détail à petite échelle et l'épargne.

Enfin, les femmes du village d'Ofua II se sont organisées en un groupement de 30 membres pour développer les compétences de leurs pairs, notamment en matière de couture, de perlage, de tricot et de tissage, de fabrication de chaussures, de coiffure et de fabrication de savon. Ce groupement de femmes propose des formations pour doter leurs sœurs non alphabétisées de la communauté de compétences leur permettant de subvenir à leurs besoins.

\section{Résultats}

- Les personnes concernées ont confiance en leurs dirigeants et continuent à faire connaître leurs préoccupations par l'intermédiaire des structures établies. Il y a une participation notable des responsables des réfugiés à la prise de décisions pour résoudre les questions qui les concernent, eux et leur communauté, ainsi qu'une plus grande visibilité des dirigeants de la communauté à travers les espaces établis.

- Les groupes de soutien des personnes ayant des besoins spécifiques ont économisé de l'argent et y ont contribué dans le cadre de leur Association villageoise d'épargne et de prêt. Ils continuent également à offrir des services de conseil et de sensibilisation aux autres personnes ayant des besoins spécifiques sur leurs droits et obligations.

- Le groupe de femmes d'Ofua II a acquis sept machines à coudre et d'autres kits pour former d'autres femmes. À ce jour, le groupe a formé plus de 16 femmes à la couture et cinq femmes à la fabrication de chaussures. Les formations continues comprennent la couture (pour 10 femmes) et le perlage, le tricot et le tissage (56 femmes). Les membres du groupe versent l'équivalent d'environ 20000 shillings ougandais pour l'adhésion, 500 pour l'inscription et 1000 par semaine. Le groupe décide conjointement de la manière de dépenser les fonds.

\section{Partenaire(s) :}

Conseil danois pour les réfugiés

Cabinet du Premier ministre

Pour de plus amples informations, contacter:

Jael Chunge : chunge@unhcr.org

Kalsum ljoru : ijoru@unhcr.org

\section{Étude de cas en Ouganda : Initiative communautaire de consolidation de la paix}

\footnotetext{
À la suite du meurtre par des réfugiés d'un jeune ougandais dans le camp de réfugiés de Rhino Camp (Eden Zone), les relations entre réfugiés et membres des communautés d'accueil sont devenues tendues et menacées. II était I par conséquent nécessaire d'aborder et de résoudre la situation, et de rétablir la paix qui existait entre les deux communautés avant ce malheureux incident. L'Initiative communautaire de consolidation de la paix est née de ces circonstances.
}

Cette initiative a impliqué des dialogues communautaires de consolidation de la paix menés par les dirigeants des réfugiés d'Eden Zone et ceux de la communauté d'accueil. Quatre sessions dialogues ont été menées - deux avec la communauté d'accueil et deux avec la communauté de réfugiés. Au début du dialogue, les suspects avaient été arrêtés. Au cours des sessions, les proches du défunt ont dénoncé le crime, tout en acceptant que la condamnation de tous les réfugiés serait injuste et en mettant en garde les ougandais contre les représailles. 


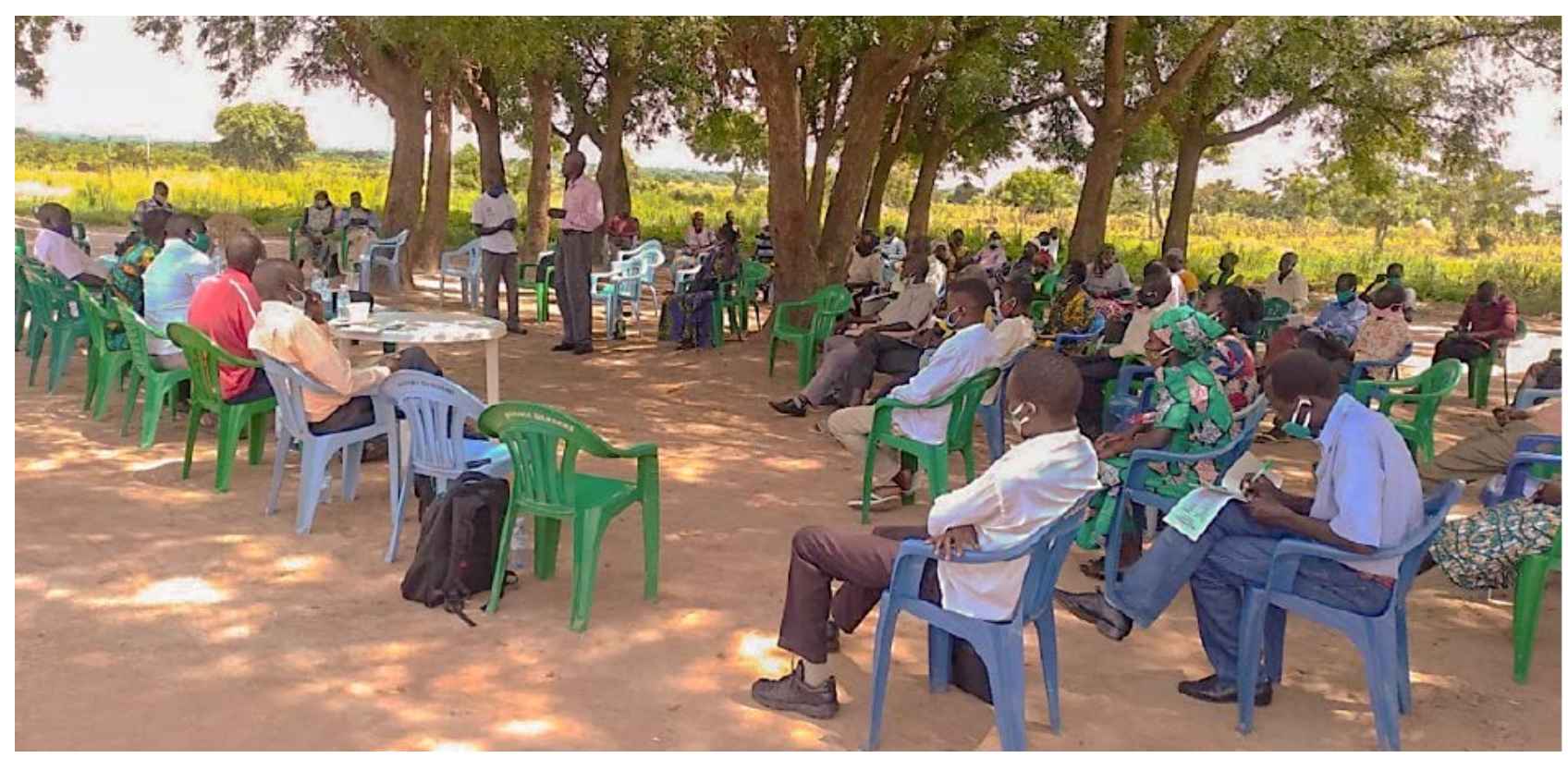

Le dialogue a permis aux membres des communautés de réfugiés d'exprimer leurs remords et de demander pardon aux membres d'accueil lésés. Les membres de la communauté des réfugiés ont assumé la responsabilité de l'incident, soulignant aux membres de la communauté d'accueil qu'ils avaient fui leur pays pour leur propre sécurité, que le meurtre est aux antipodes de la légalité et que tous les réfugiés n'étaient pas impliqués dans ce crime.

II a été décidé que les réfugiés et les ressortissants devraient coexister et continuer à partager les ressources disponibles. Les réfugiés qui avaient acquis ou loué des terres étaient encouragés à reprendre la production agricole, la collecte de bois de chauffage, la collecte de matériaux de construction, etc. Les deux communautés résolues ont mis fin à cet incident malheureux et ont entamé un nouveau chapitre ensemble.

\section{Partenaire(s) :}

Community Technology Empowerment Network

Conseil danois pour les réfugiés

Development Literacy Partner International

Conseil norvégien pour les réfugiés

Cabinet du Premier ministre

Pour de plus amples informations, contacter :

Jael Chunge : chunge@unhcr.org

Ejoyi Geoffrey Bakole : ejoyi@unhcr.org

\section{Pratique émergente en Ouganda : Initiative tricycles}

es personnes ayant des besoins spécifiques dans le camp de réfugiés de Bidibidi rencontrent toujours des difficultés pour accéder à la nourriture en raison de l'éloignement des points de distribution dans le camp. Certains parcourent _jusqu'à cinq kilomètres pour atteindre les centres de distribution et sont obligés de vendre une partie de leurs rations pour organiser la collecte et la livraison. Afin d'alléger la charge de transport des personnes ayant des besoins spécifiques, le HCR a lancé une initiative tricycles pour garantir un accès continu et sûr à l'aide alimentaire pour les personnes particulièrement vulnérables parmi celles ayant des besoins spécifiques grâce à un mécanisme de transport abordable et durable.

Ce projet vise un double objectif : 1) faciliter les services de transport gratuits et sûrs pour les familles particulièrement vulnérables des points de distribution de nourriture à leur lieu d'hébergement à l'intérieur de la zone d'installation, et 2) soutenir les possibilités de génération de revenus pour certaines associations d'épargne et de prêt volontaires les jours de distribution de produits non alimentaires. 


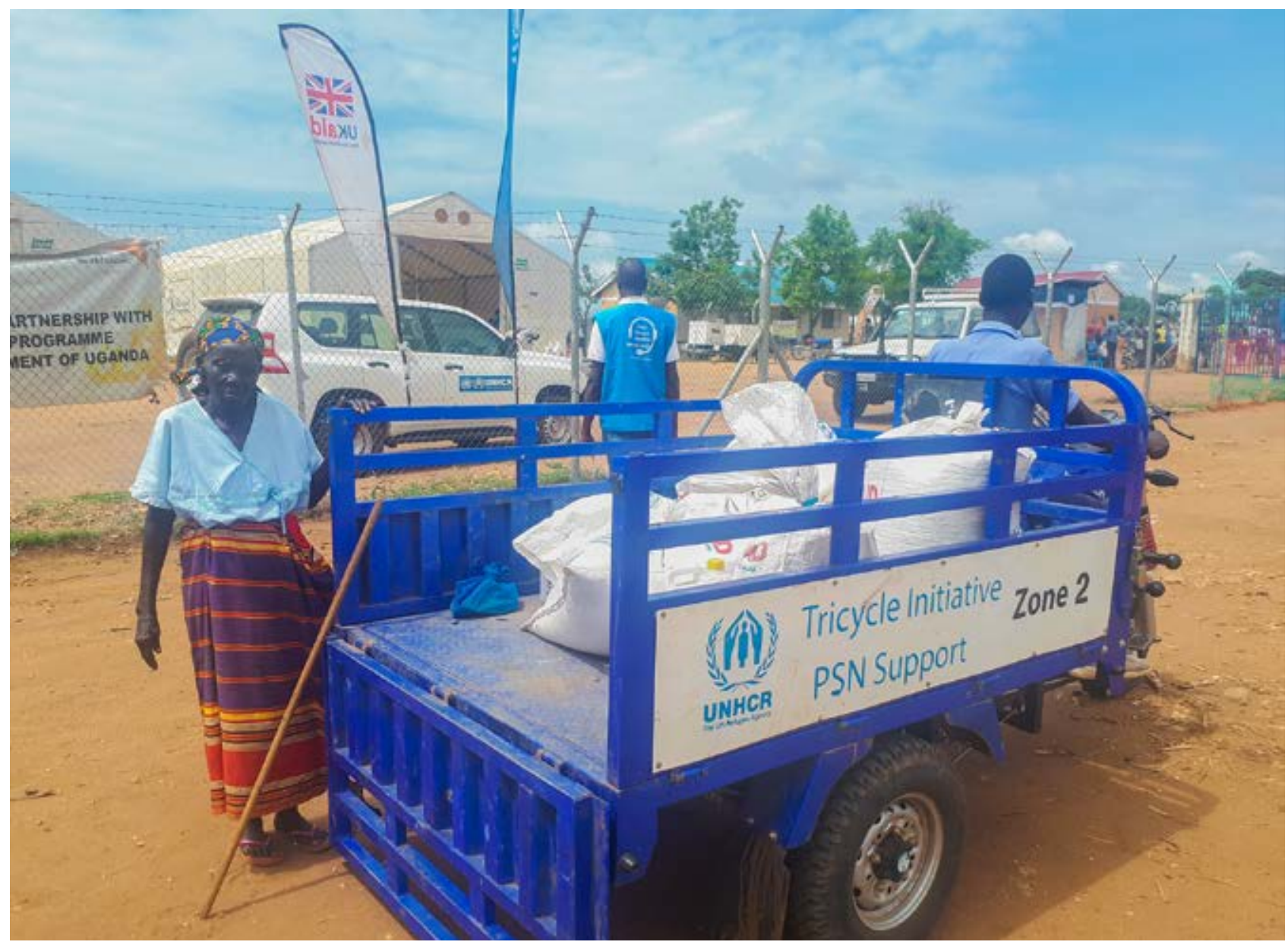

Pour atteindre ces objectifs, le HCR a alloué 20 tricycles au camp de réfugiés de Bidibidi. Les tricycles ont été distribués par zone à des associations d'épargne et de prêt volontaires sélectionnés, en fonction des besoins des zones. L'attribution a été faite en tenant compte de la taille et de la typologie de la zone, ainsi que de la distance par rapport aux points de distribution de nourriture et du nombre d'individus particulièrement vulnérables ayant besoin de transport dans chaque zone. Les partenaires de protection ont soutenu la désignation de ces personnes par une approche communautaire, en engageant la structure communautaire (secrétaire aux personnes ayant des besoins spécifiques du Conseil de protection des réfugiés). Les associations d'épargne et de prêt volontaires ont également été sélectionnées parmi plus de 1000 groupes actifs grâce à une approche interinstitutions impliquant tous les partenaires de la protection et des moyens de subsistance ainsi que le cabinet du Premier ministre.

Les groupes sélectionnés ont été évalués sur la base de l'expérience, du niveau et des preuves de transactions d'épargne et de prêts ainsi que de la motivation du groupe à servir les personnes ayant des besoins spécifiques, en interrogeant tous les membres du groupe. Certains groupes réunissent soit exclusivement des femmes, soit exclusivement des hommes, tandis que d'autres sont mixtes, selon le modèle commercial de l'association d'épargne et de prêt volontaires ; toutefois, un mélange d'hommes et de femmes a été sélectionné pour l'initiative.

Les associations sélectionnées ont été formées à l'utilisation des tricycles et deux membres par groupe ont eu un permis de conduire. La formation et l'obtention des permis de conduire ont été facilitées par Dan Church Aid. Les associations ont bénéficié d'un soutien pour le carburant et les réparations et services mineurs pendant une période initiale de trois mois, ce qui leur a donné le temps d'acquérir une forme d'autonomie. Un mémorandum d'accord a été signé entre les associations d'épargne et de prêt volontaires, Dan Church Aid, le HCR, le cabinet du Premier ministre et IRC, stipulant l'objectif de cette initiative et les rôles et responsabilités de toutes les parties.

Les 20 tricycles ont été remis aux groupes dans différents quartiers de la zone d'installation de Bidibidi. Ils fonctionnent bien et aident les personnes particulièrement vulnérables lors de la distribution de nourriture dans leurs zones respectives à transporter gratuitement leurs provisions jusqu'à leurs lieu d'hébergement, pendant une moyenne de cinq jours ouvrables par distribution. Le reste du mois, ils exercent des activités génératrices de revenus dans le cadre des conditions générales du mémorandum d'accord qu'ils ont signé. L'initiative a également eu un effet positif sur la génération de revenus des associations d'épargne et de prêt volontaires. 


\section{Résultats}

Les propos suivants d'un membre de l'association résume les résultats obtenus par l'initiative jusqu'à présent : « quand nous avons lancé l'activité des tricycles, lors de nos séances hebdomadaires, les membres économisaient 3000 shillings ougandais. Nous prévoyons de faire passer les économies à 4000 shillings ougandais pour notre prochain cycle annuel qui commence en octobre 2020, neuf mois après le début de l'initiative tricycles Malgré la situation marquée par le coronavirus et la restriction des mouvements, nous parvenons toujours à créer des emplois dans la zone d'installation et le projet a considérablement augmenté les revenus générés par l'association. Avant, le montant le plus élevé de prêt était de 500000 shillings ougandais. À présent, nous pouvons maintenant accorder des prêts jusqu'à 1000000 shillings ougandais. Cela aidera les membres à penser à des projets plus importants et à augmenter leurs possibilités de manière exponentielle».

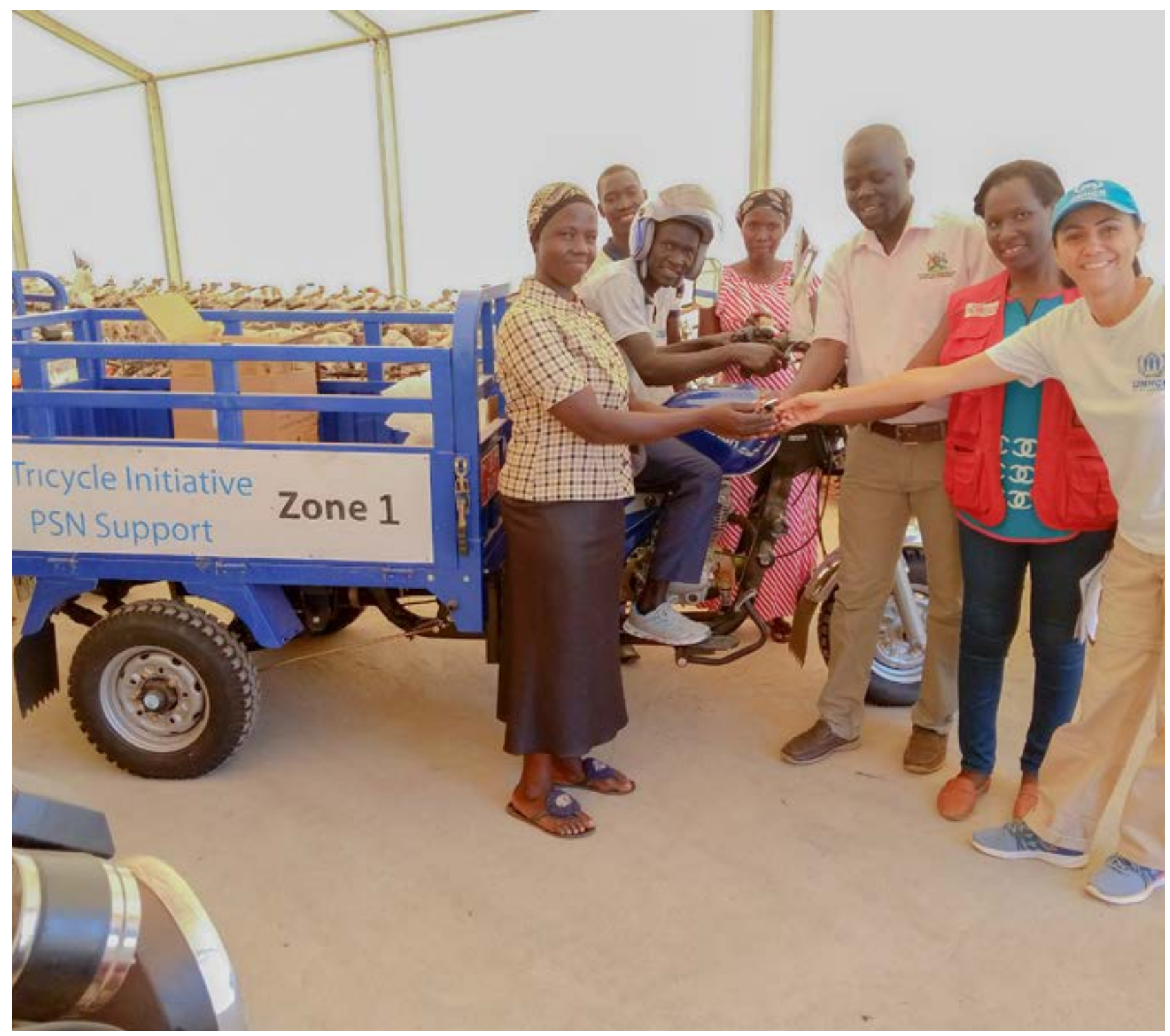

Partenaire(s) :

Dan Church Aid

Comité international de secours

Cabinet du Premier ministre

Pour de plus amples informations, contacter:

Elham Baghdadi : baghdad@unhcr.org

Vincent Bukenya : bukenyav@unhcr.org 


\section{Étude de cas au Soudan du Sud : Réseau de protection communautaire pour les personnes déplacées}

'éclatement du conflit au Sud-Soudan a entraîné le déplacement de nombreuses personnes vers les centres urbains. Malgré la signature d'un accord de paix, la peur règne toujours parmi les populations déplacées. Nombre de ces personnes déplacées craignent pour leur vie et rechignent à rentrer chez elles. La majorité des personnes déplacées sont restées vulnérables après que leurs maisons aient été vandalisées et que leurs moyens de subsistance aient été perturbés par le conflit armé.

Pour soutenir les personnes déplacées, un réseau communautaire de protection a été mis en place en tant que structure de protection clé émanant de la communauté ; il devrait favoriser l'engagement de ses membres dans l'évaluation ou la distribution de l'aide humanitaire, l'identification des ménages vulnérables parmi les personnes déplacées et le partage des informations dans neuf sites qui les accueillent dans la ville de Yei. La nouvelle structure communautaire a été mise en place, tout en renforçant les structures locales existantes en tant qu'initiative de protection. Cette approche visait à garantir l'appropriation par la communauté et la durabilité de l'initiative. Le réseau de protection communautaire constitue un forum permettant aux membres de la communauté de s'entraider dans leur travail quotidien, et de développer et diffuser les meilleures pratiques, directives et stratégies à l'usage de leurs membres.

Dans le cadre de ce réseau, une formation continue sur le terrain a été dispensée aux structures communautaires afin de renforcer les compétences et d'améliorer la qualité du soutien apporté aux personnes déplacées vulnérables et aux communautés d'accueil. Afin d'optimiser l'utilisation des ressources disponibles et d'éviter le double ciblage et la duplication des efforts, le Comité des méthodistes unis pour l'aide humanitaire a collaboré avec d'autres acteurs humanitaires tout au long de cette initiative. Les activités proposées dans le cadre du projet ont été menées dans neuf sites de déplacés établis avec l'aide des pouvoirs publics de l'État. Les structures établies ont joué un rôle majeur dans la sensibilisation et la conscientisation des communautés, l'identification des bénéficiaires, le signalement des violations de la protection, et la réponse aux victimes touchées qui cherchaient un soutien et la justice pour les atrocités commises.

Le réseau communautaire a permis d'aboutir aux résultats suivants :

- Un système fonctionnel de suivi de la protection communautaire, permettant une meilleure prise de conscience des risques de violence sexuelle et fondée sur le genre, des canaux de signalement clairs et des procédures de gestion des cas en temps utile et dans la dignité pour les survivants de ces violences

- Mécanisme de collecte de données fonctionnel et efficace, avec validation et respect de la confidentialité.

- Groupes de soutien fonctionnels établis pour les personnes ayant des besoins particuliers afin d'assurer l'autoprotection et l'autogestion de la communauté.

Partenaire(s) :

Comité des méthodistes unis pour l'aide humanitaire

Mission to Alleviate Suffering in South Sudan

Pour de plus amples informations, contacter:

Wamutshilani Nkala : nkalaw@unhcr.org 


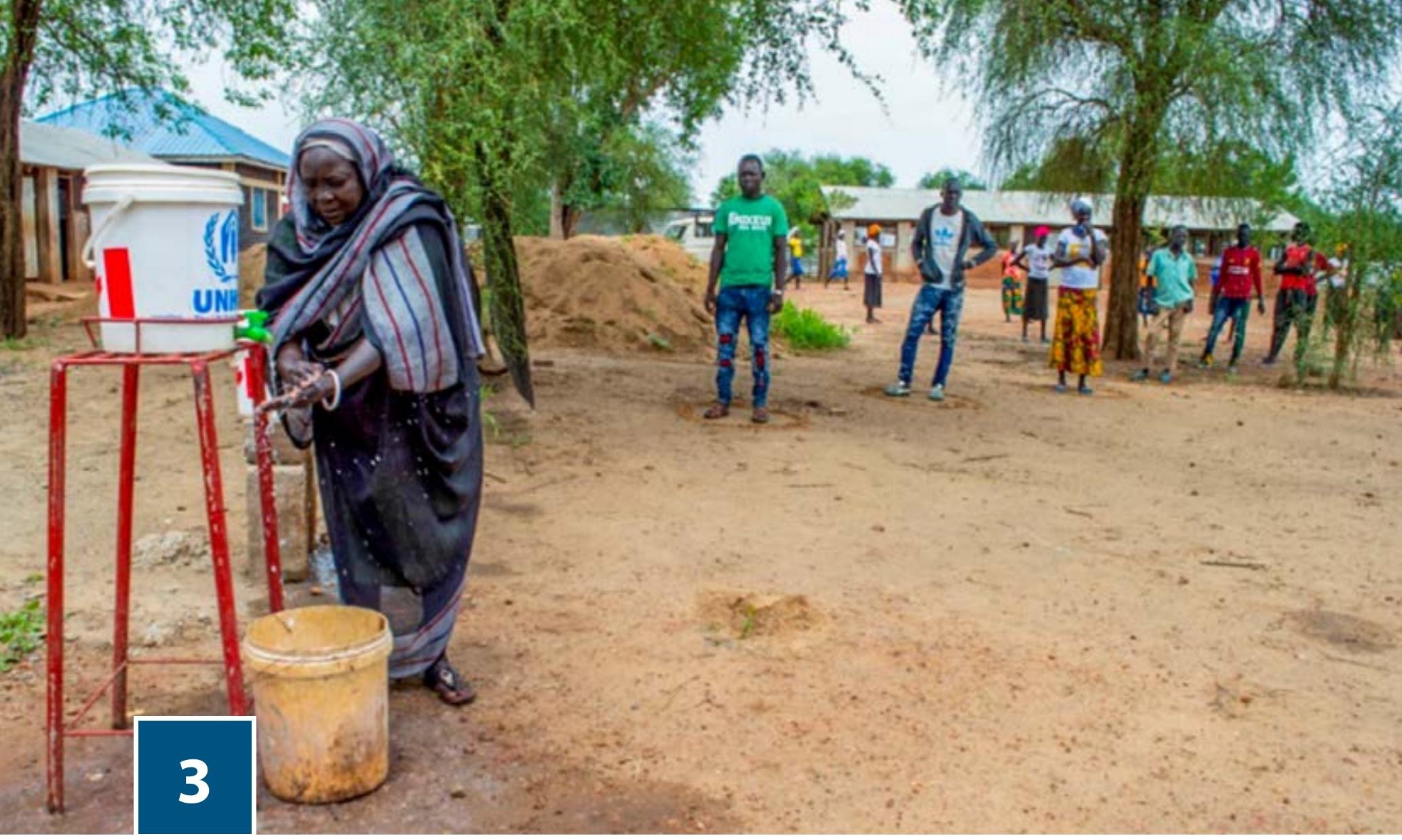

\section{ÉGALITÉ DES FEMMES ET DES HOMMES}

\section{Pratique prometteuse en Éthiopie : Volontaires pour l'aide aux réfugiés à Addis-Abeba}

S i la majorité des réfugiés en Éthiopie résident dans des camps prévus à cet effet, environ 30000 réfugiés de différentes nationalités résident dans la capitale, Addis-Abeba. Certains réfugiés se rendent à Addis-Abeba sur recommandation des camps de réfugiés pour des raisons de santé, de sécurité, de violence sexuelle et fondée sur le genre et d'autres raisons humanitaires qui ne peuvent être traitées dans les camps. La barrière de la langue dans les centres urbains empêche souvent les réfugiés d'accéder aux divers services nationaux, notamment les services juridiques, de santé, d'éducation et autres fournis par le gouvernement.

Les volontaires pour l'aide aux réfugiés sont des bénévoles qui vont à la rencontre de leurs collègues réfugiés ayant des besoins spécifiques (tels que les personnes âgées, les personnes handicapées, les survivants de la violence sexuelle et fondée sur le genre et les LGBTI) et leur fournissent des informations précises, mènent des actions de sensibilisation sur des questions importantes et aident à identifier et à orienter les réfugiés vers les prestataires de services appropriés pour tout besoin de protection, de santé, d'enregistrement et autres. Compte tenu de leur connaissance des contextes culturels, socioéconomiques et religieux respectifs, et de leur bonne compréhension des besoins et des lacunes des réfugiés en matière de protection, les volontaires jouent également un rôle essentiel dans la prévention et le traitement de la désinformation et de l'incompréhension de la population réfugiée concernant le HCR et les services des partenaires.

L'approche d'intervention consiste à sélectionner les volontaires pour l'aide aux réfugiés dans la communauté de manière structurée, inclusive et transparente, en tenant compte des dimensions d'âge, de genre et de diversité ainsi que de la position et des réseaux des volontaires dans la communauté, avec le soutien technique du HCR et de son homologue gouvernemental, I'Administration pour les réfugiés et les rapatriés. Au total, 49 volontaires pour l'aide aux réfugiés (24 hommes et 25 femmes) ont été sélectionnés dans la région des Grands Lacs, au Soudan, au Yémen, au Sud Soudan, en Érythrée, en Somalie et parmi des personnes d'autres nationalités vivant à Addis-Abeba.

Avec les conseils techniques du HCR, les volontaires pour l'aide aux réfugiés sont divisés en trois domaines thématiques: la protection de l'enfance, la protection générale et la santé, et effectuent une série de tâches pour identifier les réfugiés 
ayant des besoins spécifiques. Ces tâches vont de la recherche de familles d'accueil pour les enfants séparés et non accompagnés, à l'aide à la traduction dans les établissements de santé, en passant par l'accompagnement de ces patients vers des prestataires de services d'autres secteurs, notamment la police et le secteur juridique, selon les besoins.

Les volontaires pour l'aide aux réfugiés reçoivent une formation et des mises à jour intermittentes sur les principes « ne pas nuire», la confidentialité, l'inclusion et l'impartialité. Ils sont évalués périodiquement sur ces questions lors de visites de supervision mensuelles, et leur engagement est examiné pendant cette période ainsi que lors des réunions mensuelles de gestion des cas et des réunions générales trimestrielles des volontaires pour l'aide aux réfugiés qui offrent une plateforme à tous les volontaires pour partager leurs défis et leurs succès, et pour renforcer leur esprit d'équipe. La formation des volontaires pour l'aide aux réfugiés couvre les concepts de base de la protection contre l'exploitation et les abus sexuels et de la lutte contre la fraude, et chaque volontaire signe un code de conduite.

Les volontaires pour l'aide aux réfugiés sont chargés d'identifier quatre cas par mois composés de personnes ayant des besoins spécifiques qui requièrent l'attention du HCR ou d'un partenaire d'exécution. Les cas identifiés font l'objet d'un suivi, et un retour d'information sur les mesures prises est fourni aux volontaires pour l'aide aux réfugiés.

\section{Résultats}

Les résultats sont mesurés sur la base des rapports mensuels des personnes relevant de la compétence du HCR atteintes, des personnes orientées vers les prestataires de services, du nombre de suivis effectués et des besoins satisfaits. Les commentaires des personnes relevant de la compétence du HCR et des superviseurs immédiats sont enregistrés et classés dans le dossier physique de chaque volontaires pour l'aide aux réfugiés ainsi que dans la base de données ProGres du HCR.

Partenaire(s) :

Administration pour les réfugiés et les rapatriés

Development and Inter-Church Aid Commission

Service jésuite des réfugiés

Pour de plus amples informations, contacter:

Nunu Aliyi : aliyi@unhcr.org

\section{Étude de cas au Kenya : Égalité des femmes et des hommes dans l'approvisionnement en eau, assainissement et hygiène (WASH)}

es services WASH du camp de réfugiés de Kakuma et de la zone d'installation de Kalobeyei sont spécialement organisés pour être fournis à toutes les personnes relevant de la compétence du HCR , y compris les femmes, les hommes, les

filles et les garçons, dans des conditions d'égalité et d'équité. Un accent particulier est mis sur les femmes et les filles, car elles sont plus susceptibles d'être laissées pour compte et sous-représentées dans les approches conventionnelles de participation communautaire.

Au niveau des camps, les bénéficiaires de l'aide en matière de WASH sont identifiés par des équipes multifonctionnelles composées du HCR, du Secrétariat aux affaires des réfugiés, des partenaires d'exécution en matière de santé et d'une série d'autres partenaires. La priorité est donnée aux ménages dirigés par des femmes.

Au niveau des blocs, les comités WASH (structures communautaires représentatives du secteur WASH) sont formés pour identifier et coordonner les questions WASH au sein de leurs blocs respectifs. Le Conseil norvégien pour les réfugiés a assuré une représentation diversifiée des dirigeants au sein des comités WASH, y compris la parité femmes-hommes parmi les représentants. Les candidatures féminines sont également prioritaires dans le recrutement du personnel de sensibilisation communautaire WASH.

Partenaire(s) :

Conseil norvégien pour les réfugiés

Pour de plus amples informations, contacter :

Moe Miyahara : miyahara@unhcr.org

Oscar Nabiswa : nabiswa@unhcr.org

John Wagacha Burton : burtonj@unhcr.org 


\section{Étude de cas en Ouganda : Plan de travail/tableau d'indicateurs sur l'âge, le sexe et la diversité}

'application d'une approche fondée sur l'âge, le sexe et la diversité (AGD) dans notre travail est essentielle pour garantir que nos interventions sont sûres et accessibles à tous et qu'elles ne laissent aucune place à la discrimination. Cette approche implique la prise en compte des besoins et des points de vue distincts des femmes, des hommes, des filles et des garçons d'origines diverses, y compris les personnes handicapées, les personnes ayant des orientations sexuelles et des identités de genre différentes et les minorités ethniques, religieuses et linguistiques ou les groupes autochtones, dans les processus d'évaluation, de planification, de mise en œuvre et de suivi.

Afin de s'assurer que tous les collègues et secteurs du HCR appliquent une approche AGD dans leur travail, les équipes de la protection communautaire et du programme ont élaboré des plans d'action et de travail nationaux AGD pour l'opération. Le plan de travail AGD mis en œuvre à l'heure actuelle est basé sur le plan d'action national AGD qui a été élaboré en 2019. Le plan d'action national AGD a consolidé les plans d'action sur le terrain qui ont été élaborés après un atelier d'examen en profondeur en 2018, et contient une liste de défis, d'activités sur le terrain et d'activités au niveau national pour mettre en œuvre efficacement la politique AGD du HCR. Ce plan d'action a été conçu par le biais de consultations avec les responsables de secteur à Kampala, le personnel de protection sur le terrain et les partenaires.

Le plan de travail AGD s'inspire du plan d'action AGD, mais ressemble à un tableau d'indicateurs, introduisant des indicateurs de niveau de résultat et de production, des bases de référence et des objectifs. Cela nous aidera à contrôler plus efficacement la mise en œuvre des activités prévues dans le plan de travail. La version finale du plan/tableau sera produite à l'issue d'autres consultations avec les responsables des secteurs (au sein du HCR et en dehors) et les collègues sur le terrain. II s'agit d'un effort conjoint entre les collègues de la protection communautaire et du programme.

L'impact du plan de travail AGD pour 2020-2021 reste à être évalué. Cependant, il devrait avoir un impact sur la manière dont les services sont fournis en Ouganda, et sur la manière dont la politique AGD du HCR est mise en œuvre. L'application efficace d'une approche AGD dans le travail des partenaires permettrait d'accroître la sécurité et l'accès dans de bonnes conditions aux services pour tous et de donner des moyens d'action aux groupes marginalisés et privés de leurs droits.

Le processus de travail avec les partenaires et le personnel du HCR a déjà permis de faire connaître cette politique. Le partenariat entre la protection et le programme a été très positif. L'implication d'un administrateur de programmes hors classe en a fait une initiative du HCR, plutôt qu'une initiative de protection.

Partenaire(s) : divers

Pour de plus amples informations, contacter:

Sam Mosallai : mosallai@unhcr.org

Yokolwasa : iwasa@unhcr.org

Clare Askew : askew@unhcr.org

\section{Étude de cas en Ouganda : Plans de travail fondé sur l'âge, le sexe et la diversité pour les partenaires d'exécution}

$\int d$ ne approche fondée sur l'âge, le sexe et la diversité favorise une prise en compte nuancée des réalités, des points de vue et des besoins des femmes, des hommes, des filles et des garçons d'origines diverses dans les processus d'évaluation, de planification, de mise en œuvre et de suivi. Les populations d'intérêt comprennent également les personnes handicapées, les personnes ayant des orientations sexuelles et des identités de genre diverses et les minorités ethniques, religieuses et linguistiques ou les groupes autochtones.

Pour favoriser l'application d'une approche AGD dans le travail de ses partenaires d'exécution, le HCR a fait du respect de sa politique AGD une exigence obligatoire pour tous les partenaires d'exécution. L'équipe de protection communautaire, avec le soutien de collègues du programme, travaille depuis lors avec de nouveaux partenaires d'exécution pour renforcer leurs capacités sur l'approche AGD.

Avant ce renforcement des capacités, les modèles d'accords de partenariat de projet en cours du HCR contenaient peu de 
références à I'AGD, sans aucune mention de l'attente que les partenaires d'exécution se conforment à la politique du HCR en la matière. Par conséquent, les partenaires n'étaient pas au courant de cette politique.

Pour remédier à cette situation, l'équipe de protection communautaire a travaillé en étroite collaboration avec les collègues du programme tout au long du processus de sélection des partenaires pour 2020. Les collègues de la protection communautaire ont pris part à toutes les réunions initiales avec les nouveaux partenaires, ont fait des présentations sur la politique AGD, ont informé les partenaires de leur obligation de se conformer à la politique et leur ont fourni des paquets de soutien AGD. En outre, une référence détaillée à l'approche AGD a été incluse dans tous les nouveaux accords de partenariat de projet et lettres de motivation, et il a été convenu que tous les partenaires élaboreraient des plans de travail AGD individuels et spécifiques à chaque secteur. En outre, une session de formation d'une demi-journée a été organisée, au cours de laquelle les partenaires ont été davantage sensibilisés à la politique AGD, ainsi qu'au Comité permanent interinstitutions sur le genre avec marqueur d'âge. Les partenaires ont également reçu des conseils sur l'élaboration de plans de travail sectoriels pour l'année suivante, afin d'assurer l'intégration effective de l'égalité des femmes et des hommes et de la responsabilité envers les personnes touchées dans toutes les activités des partenaires. Ces plans de travail contiennent des activités, des indicateurs, des personnes de référence et des échéances. Les progrès réalisés dans la mise en œuvre de ces plans de travail seront suivis lors des examens de performance en milieu et en fin d'année.

L'inclusion de l'AGD dans les accords de partenariat de projet pour 2020, lintroduction d'exigences de rapport obligatoires et l'élaboration de plans de travail AGD spécifiques aux secteurs des partenaires devraient avoir un impact sur la prestation de services dans les situations de réfugiés en Ouganda. L'application efficace d'une approche AGD dans le travail des partenaires permettrait probablement d'accroître la sécurité et l'accès significatif aux services pour tous, et renforcera I'autonomie des groupes marginalisés et privés de leurs droits. Ce processus de collaboration entre le personnel du HCR et ses partenaires a déjà permis d'accroître considérablement la sensibilisation à la politique AGD.

Partenaire(s) : divers

Pour de plus amples informations, contacter :

Sam Mosallai : mosallai@unhcr.org

Yoko Iwasa : iwasa@unhcr.org 


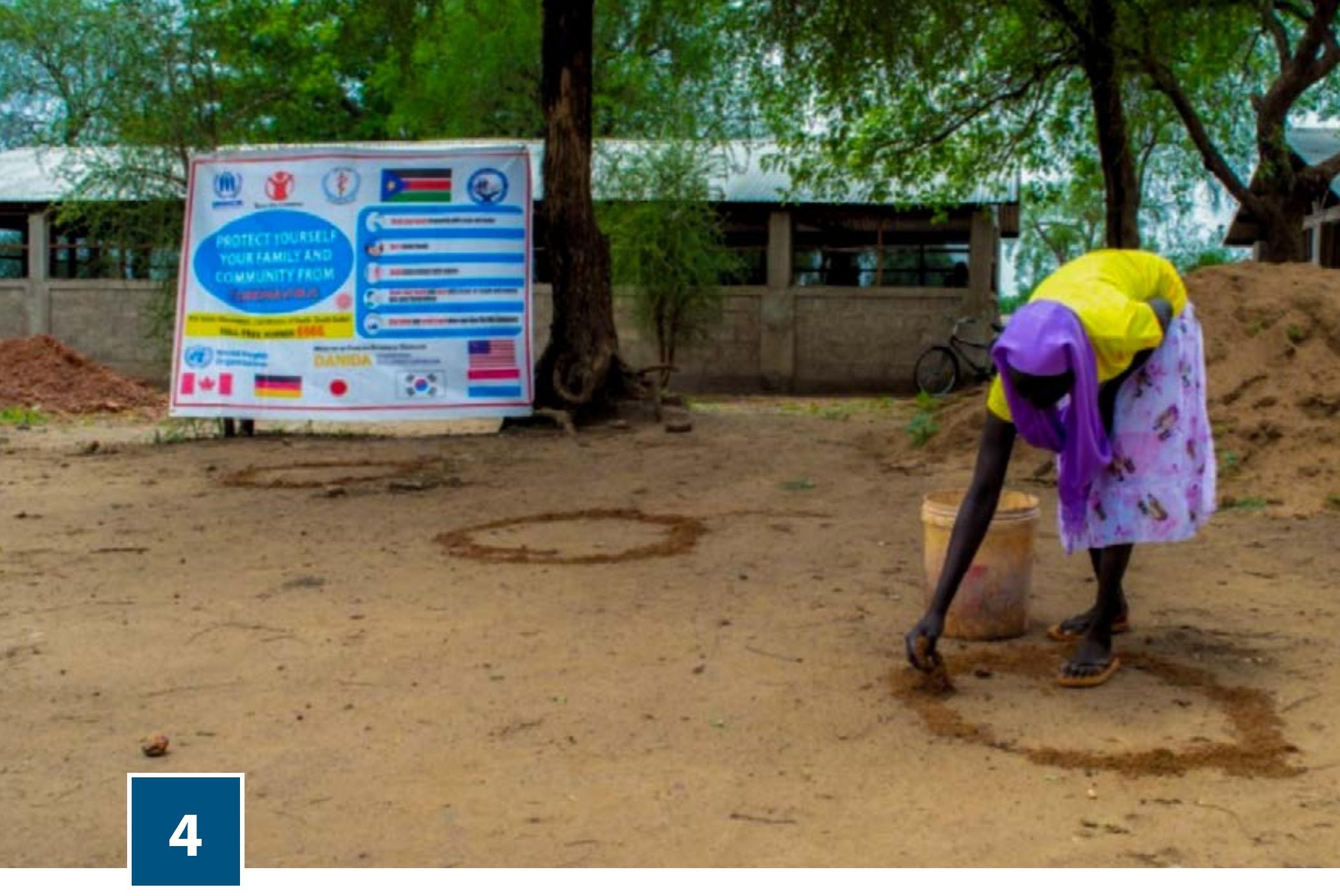

\section{VIOLENCE SEXUELLE ET FONDÉE SUR LE GENRE/ATTÉNUATION DES RISQUES/RÉPONSE}

\section{Pratique émergente en Éthiopie : Moulin communautaire exploité par une association de femmes}

$\circlearrowleft$ ne évaluation des besoins a permis d'identifier un moulin communautaire comme étant important, étant donné que les réfugiés étaient obligés de parcourir de longues distances à pied jusqu'à la communauté d'accueil pour moudre leurs rations. Cela a non seulement coûté cher à la communauté des réfugiés, mais a également entraîné des risques accrus de violence sexuelle et fondée sur le sexe pour les femmes et les filles. Pour atténuer cette situation tout en permettant aux femmes des camps de réfugiés de participer à des projets de subsistance qui les aideraient à générer des revenus durables, le HCR a construit et équipé un moulin communautaire et l'a remis à une structure communautaire - I'« Association des femmes ». L'association des femmes a été choisie en consultation avec les représentants de la communauté. L'association comprend toutes les nationalités et ethnies, travaillant en harmonie et promouvant la coexistence pacifique.

Outre la construction du moulin, cette intervention implique la formation des membres de l'association des femmes par le HCR et l'Administration pour les réfugiés et les rapatriés sur le fonctionnement du moulin et la gestion des finances qu'il génère. En outre, le HCR a fourni une assistance en matière de carburant et de main-d'œuvre pendant un an. L'Association des femmes a travaillé en étroite collaboration avec le HCR jusqu'à ce qu'elle deviennent totalement indépendante. Des conseils et un soutien sont toujours fournis en cas de besoin.

\section{Résultats}

Les communautés de réfugiés et d'accueil ont maintenant accès à un moulin à faible coût et bien situé. L'implantation du moulin au sein de la communauté des réfugiés réduit les risques de protection et de sécurité pour les femmes, les jeunes filles et les jeunes. L'Association des femmes, totalement autonome, a généré des bénéfices, ce qui l'a conduite à décider 
d'inclure davantage de femmes de la communauté des réfugiés et d'étendre le projet en se lançant dans des initiatives de salons de beauté et de restauration dans le camp. L'Association des femmes examine actuellement l'expansion de l'entreprise. Le moulin a également créé des emplois salariés pour plus de quatre personnes concernées au sein du camp. Les femmes qui participent au projet ont amélioré leurs moyens de subsistance et contribuent financièrement à leur ménage et à leur famille. Le projet a attiré l'attention d'un plus grand nombre de femmes qui cherchent à participer à de tels projets de subsistance.

Partenaire(s) :

Administration pour les réfugiés et les rapatriés

Pour de plus amples informations, contacter :

Alisona Rajbanshi : rajbansh@unhcr.org

\section{Étude de cas au Kenya : Stratégies WASH sensibles au genre comme mesure de prévention de la violence sexuelle et fondée sur le genre}

$\int$

our dissuader les auteurs de la violence sexuelle et fondée sur le genre dans les camps de réfugiés, des points d'eau sont délibérément construits dans des espaces ouverts et à la portée de la communauté. En outre, l'approvisionnement en eau est assuré de 7 heures à 17 heures, plutôt que plus tard dans la soirée ou la nuit, afin de garantir la sécurité générale de la communauté. Pour atténuer les conflits aux points d'eau (robinets), les comités WASH et les responsables des réfugiés ont travaillé avec leurs communautés pour élaborer un programme communautaire de collecte d'eau organisé les matins, les midis et les soir. De plus, les latrines communes pour les nouveaux réfugiés arrivants sont conçues avec des volets internes pour protéger l'intimité et la dignité. Les membres de la communauté ont également été sensibilisés pour accompagner les mineurs et les femmes aux latrines la nuit.

Partenaire(s) :

Conseil norvégien pour les réfugiés

Pour de plus amples informations, contacter :

Moe Miyahara : miyahara@unhcr.org

Oscar Nabiswa : nabiswa@unhcr.org

John Wagacha Burton : burtonj@unhcr.org

\section{Pratique émergente au Kenya : Des maisons sûres gérées par la communauté}

e HCR et ses partenaires disposent d'un groupe de volontaires formés de la communauté des réfugiés qui ont ouvert leurs maisons pour offrir des espaces sûrs aux survivants de violence sexuelle et fondée sur le genre ayant besoin d'un abri temporaire et d'un soutien en attendant l'évaluation de leurs besoins et des solutions. Des bénévoles formés fournissent un abri dans l'espace de leur foyer et aident à orienter les survivants vers des points de service clés, tels que les établissements de santé. Cette intervention garantit que les survivants qui n'ont pas besoin d'une institutionnalisation à long terme ont toujours accès à des espaces sûrs au sein de la communauté, et que la capacité de la communauté est renforcée pour identifier de manière indépendante des solutions et un soutien.

Les victimes de violence sexuelle et fondée sur le genre reçoivent un soutien matériel, des premiers soins psychosociaux, des références aux services d'urgence et des services volontaires. Seuls les cas à faible et moyen risque sont admis dans les foyers communautaires sécurisés après qu'une évaluation de la sécurité et une planification de la sécurité aient été effectuées avec le consentement du survivant. L'accent est mis sur une approche centrée sur les survivants tout en envisageant de les admettre dans des foyers communautaires sécurisés. L'avis du bénévole du foyer d'accueil par rapport aux détails du profil particulier et aux exigences du cas est également pris en considération afin de s'assurer que le préjudice est évité tant pour le survivant que pour le bénévole de la communauté. Des réunions régulières de coordination interinstitutions et des conférences de cas ad hoc sont organisées pour déterminer les plans de cas et suivre les progrès tout au long du processus d'admission. 


\section{Résultats}

Les foyers d'accueil communautaires sûrs ont été adoptés comme un complément et un substitut aux espaces institutionnels sûrs, car ces services sont souvent limités ou inexistants. Le programme a également été utilisé pour fournir un abri sûr aux hommes et aux garçons, ainsi qu'aux personnes d'orientation sexuelle, d'identité et d'expression sexuelles diverses - des populations pour lesquelles aucun espace sûr n'est dédié à leur protection, et pour lesquelles l'accès à des abris d'urgence pose un défi.

La communauté elle-même a été en mesure de répondre aux cas qui nécessitent une sécurité urgente et un abri sûr en attendant les discussions avec les agents chargés des cas de violence sexuelle et fondée sur le genre. Lídentification et le signalement des incidents de violence sexuelle et fondée sur le genre au sein de la communauté ont également été renforcés, les bénévoles des foyers d'accueil agissant en qualité de personnes de référence auxquelles la communauté peut faire appel.

Partenaire(s) : s. o.

Pour de plus amples informations, contacter :

Aisha Ngonze : ngonzea@unhcr.org

\section{Pratique émergente au Kenya : Aidants communautaires - Abris temporaires urbains sûrs pour les survivants de la violence sexuelle et fondée sur le genre}

e HCR et la Société d'aide aux immigrants juifs ont travaillé avec des structures communautaires dans les milieux urbains de réfugiés pour constituer un groupe de réfugiés volontaires qui offrent leur propre maison comme logement sûr et temporaire aux survivants de la violence sexuelle et fondée sur le genre. Dans le quartier de Kayole à Nairobi, ces volontaires/aidants communautaires suivent au moins deux formations par an pour renforcer leur capacité à soutenir les victimes de la violence sexuelle et fondée sur le genre. Ils perçoivent également une allocation pour les soutenir dans l'hébergement d'un survivant. Les travailleurs sociaux des agences partenaires surveillent les abris des ménages et apportent un soutien supplémentaire à la fois au survivant et à la personne qui s'occupe de lui.

Les comités communautaires sont sensibilisés à l'initiative des aidants communautaires et servent de plateforme pour les identifier et les sélectionner avant leur inscription au programme. Lorsqu'il n'y a pas d'aidant communautaire disponible pour accueillir un survivant, d'autres structures communautaires (par exemple, les églises) servent de refuge aux survivants.

\section{Résultats}

Cette pratique est une solution communautaire qui souligne le fait que les communautés peuvent soutenir les survivants de la violence sexuelle et fondée sur le genre en dehors du parcours classique d'orientation vers un partenaire, qui peut ne pas être fonctionnel à des moments critiques (par exemple, la nuit). L'intervention a permis de répondre rapidement aux besoins des survivants, notamment en leur assurant l'accès à un abri sûr, à des installations sanitaires et à des postes de police, ainsi qu'à des services de conseil immédiats.

Les aidants communautaires ont développé de solides relations de travail avec les autorités - en particulier la police et les fonctionnaires du sous-comté - étant donné leurs interactions régulières avec ces acteurs lorsqu'ils s'efforcent de soutenir les survivants dans leur ménage. D'autres membres de la communauté qui ne font pas partie du groupe d'aidants communautaires se portent également volontaires pour accueillir les victimes de la violence sexuelle et fondée sur le genre.

Partenaire(s) :

Société d'aide aux immigrants juifs

Secrétariat des affaires des réfugiés

Groupe de travail sur la violence sexuelle et fondée sur le genre

Autres partenaires de lutte contre la violence sexuelle et fondée sur le genre

Pour de plus amples informations, contacter:

Jackson Karugu : karugu@unhcr.org

Daniel Mureithi : mureithi@unhcr.org 


\section{Pratique émergente au Rwanda : Initiative de prévention des grossesses d'adolescentes et du décrochage scolaire}

a grossesse des adolescentes est un problème de longue date dans le camp de Kiziba, ce qui augmente le risque de décrochage scolaire chez les adolescentes. De mars à août 2020, 22 élèves ont été identifiées comme étant enceintes et à risque d'abandonner l'école.

Un certain nombre de facteurs poussent les élèves enceintes du camp à abandonner l'école, notamment le manque de soutien familial/social pour leur réintégration dans le système éducatif, la crainte de la discrimination de la part des enseignants et des pairs à l'école et l'absence d'une structure d'atténuation des risques d'abandon scolaire en cas de grossesse chez les adolescentes. L'initiative actuelle visait donc à évaluer le défi que représente le décrochage scolaire par le biais de la cartographie; comprendre les risques de décrochage scolaire pour les adolescentes enceintes par des discussions de groupe; renforcer le soutien familial et social aux adolescentes enceintes afin de promouvoir leur réintégration dans le système éducatif, en tant que stratégie d'intervention contre la violence sexuelle et fondée sur le genre ; et enfin, assurer une transition en douceur pour le retour dans le système éducatif.

La pratique comprenait les éléments suivants :

- Un exercice complet de recensement des enfants non scolarisés, facilité par les enseignants réfugiés et le partenaire éducatif du HCR, a permis d'identifier 185 enfants âgés de 3 à 19 ans ayant abandonné l'école pour de multiples raisons.

- Discussions de groupe avec des filles, des garçons, des parents et des enseignants pour mieux comprendre les risques d'abandon scolaire des filles enceintes. Les filles ont expliqué qu'elles avaient le sentiment que leur vie était finie une fois qu'elles avaient appris qu'elles étaient enceintes. Leurs parents avaient trop honte pour les laisser retourner à l'école, et les filles craignaient d'être victimes de discrimination à l'école.

- La mise en place d'une structure communautaire composée d'enseignants réfugiés et de mécanismes de coordination de la protection des enfants (CPCM) pour organiser des visites à domicile et fournir des conseils aux familles des filles enceintes identifiées par les autorités scolaires. Cette structure communautaire a été établie en collaboration avec les unités d'éducation et de protection de l'enfance, ainsi qu'avec l'équipe de protection communautaire de Karongi.

- Des sessions de formation imminentes pour les enseignants et les CPCM (dirigées par le partenaire en charge des questions de violence sexuelle et fondée sur le genre et le partenaire santé) pour renforcer les connaissances et la capacité de communication en matière d'atténuation des risques.

\section{Résultats}

Grâce à un effort soutenu, l'année du début de l'intervention, 15 adolescentes qui avaient abandonné l'école pour cause de grossesse ont repris les cours ; certaines d'entre elles ont même obtenu d'excellents résultats aux examens nationaux.

Partenaire(s) :

World Vision International

Enseignants réfugiés

Pour de plus amples informations, contacter:

Ruizhi Zhu : zhur@unhcr.org; rwaki@unhcr.org

Speciose Buwamaria : buwamari@unhcr.org

\section{Pratique prometteuse au Rwanda : Identification des survivants et réponse par le dépistage}

B ien que l'on sache que la violence sexuelle et fondée sur le genre est un problème courant dans les milieux de réfugiés, la sous-déclaration est un problème qui empêche les survivants de recevoir la réponse multisectorielle dont ils ont besoin. Afin de favoriser l'identification proactive des victimes de violence sexuelle et fondée sur le genre et de leur permettre d'accéder à des soins complets de manière pratique, le HCR Rwanda a adopté un modèle de réponse à la violence sexuelle et fondée sur le genre axé sur des preuves, mis au point par le Population Council et le Kenyatta National Hospital, au Kenya, et évalué dans les contextes de réfugiés en Ouganda en collaboration avec le HCR. 


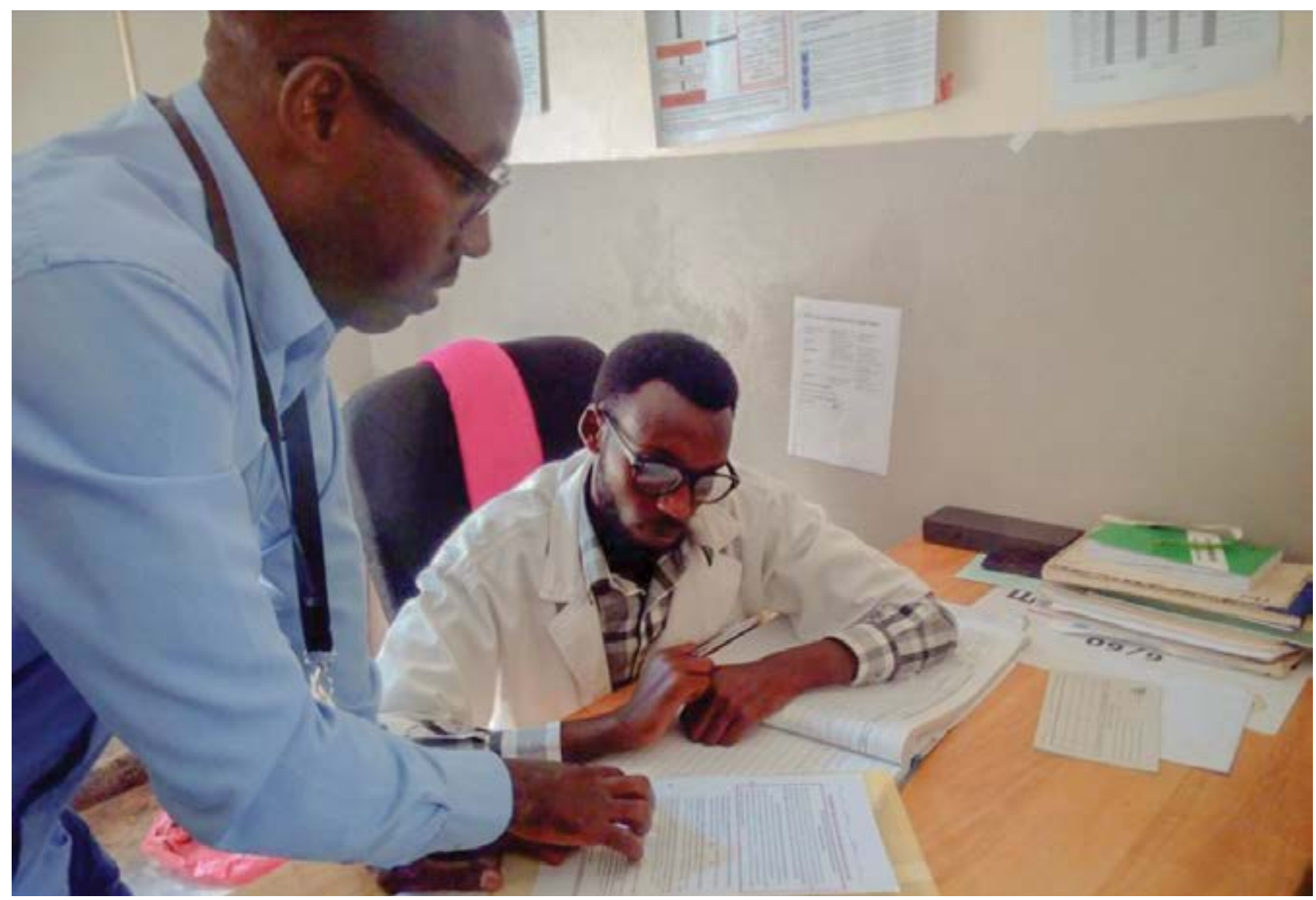

L'intervention comporte les éléments suivants:

- Session de formation et de planification des prestataires : Le HCR et Population Council ont formé les prestataires de soins de santé aux techniques de dépistage de la violence sexuelle et fondée sur le genre et ont adapté en collaboration un outil de dépistage testé utilisé pour détecter les expériences actuelles et passées de la violence sexuelle etfondée sur le genre, y compris la violence (physique, psychologique et sexuelle) exercée par un partenaire intime et le viol par une autre personne au cours de leur vie. La formation était également axée sur les concepts fondamentaux de la violence sexuelle et fondée sur le genre, la clarification des valeurs, l'éthique du dépistage, les approches centrées sur les survivants, les orientations de soutien et la documentation. Les participants à la formation comprenaient des prestataires de cinq camps au Rwanda, représentant des départements/cliniques où la confidentialité audiovisuelle pouvait être assurée (en particulier, les départements des soins ambulatoires, des maladies non transmissibles, des soins hospitaliers, des soins prénataux, de la maternité, du VIH et de la nutrition). La session a également servi de forum pour travailler en collaboration avec les prestataires participants afin d'adapter l'ensemble du processus de dépistage aux réalités du contexte de leur établissement de santé.

- Dépistage et orientation : Des prestataires de soins de santé qualifiés (infirmières, nutritionnistes, conseillers sociaux, sages-femmes) ont administré l'outil de dépistage rapide dans le cadre de consultations privées et individuelles avec toutes les clientes âgées de 14 ans et plus se présentant pour des services de santé dans les cliniques/départements respectifs. Les clientes dont le résultat du dépistage était positif ont été orientées (avec leur consentement) vers des centres de soins complets pour les violence sexuelle et fondée sur le genre, disponibles sur place dans tous les établissements de santé participants. Pour renforcer l'orientation, les appels téléphoniques entre les prestataires ont été utilisés lorsque l'accompagnement des survivantes dans les centres de soins pour la violence sexuelle et fondée sur le genre n'était pas possible.

- Collecte de données : Les cliniques participantes utilisent deux modèles de rapport mensuel pour enregistrer le nombre de clientes examinées, survivantes identifiées, survivantes référées pour des soins complets, et survivantes recevant des soins dans les centres de soins complets pour les violence sexuelle et fondée sur le genre. Les incidents par type de violence sexuelle et fondée sur le genre, les observations, les défis et les besoins sont également saisis et les données sont ventilées pour les femmes et les filles afin d'améliorer les programmes.

Le dépistage de la violence sexuelle et fondée sur le genre dans les camps de réfugiés au Rwanda a commencé en août 2017 dans les camps de Gihembe et Nyabiheke, et en juillet 2019 dans les camps de Mahama, Kigeme et Mugombwa, et se poursuit depuis lors. Les prestataires de soins de santé qui travaillent dans ces camps sont formés pour dépister systématiquement les clientes afin de détecter le plus grand nombre possible de survivantes le plus tôt possible. 


\section{Résultats}

- En 2017, les établissements de santé participants dans deux camps du Rwanda ont connu une augmentation de $300 \%$ de la proportion de survivantes identifiées et ont eu accès à des soins complets liés à la violence sexuelle et fondée sur le genre dans les deux mois suivant le début de l'intervention de dépistage.

- En 2019, dans les cinq camps de réfugiés participants au Rwanda, 326 personnes ont été identifiées comme étant survivantes de la violence sexuelle et fondée sur le genre par dépistage, et 276 ont été orientées vers les services d'intervention compétents en la matière.

- En 2019, les établissements de santé participants dans les camps de réfugiés de quatre pays réunis (dont le Rwanda) ont connu une augmentation de $129 \%$ de la proportion de survivantes identifiées et ont eu accès à des soins complets liés à la violence sexuelle et fondée sur le genre dans les six mois suivant le début du dépistage.

- Dans les 5 camps du Rwanda où le dépistage systématique de la violence sexuelle et fondée sur le genre est effectué, les partenaires de la réponse en la matière signalent une augmentation soudaine des cas reçus, qui serait liée à l'augmentation significative de la détection des survivantes et de l'accès aux soins. Les données qualitatives recueillies auprès des clients et des prestataires du centre de santé indiquent que les femmes et les filles savent maintenant qu'en plus du partenaire/agence de lutte contre la violence sexuelle et fondée sur le genre, elles peuvent également signaler les incidents qui y sont liés au centre de santé. Cela a contribué à augmenter le nombre de cas de violence sexuelle et fondée sur le genre détectés.

- Outre le Rwanda, l'intervention a été reproduite avec succès par le HCR et ses partenaires d'exécution au SudSoudan, en Ouganda et en Zambie.

- En collaboration avec Population Council, le HCR a organisé une première série de réunions avec les partenaires de l'éducation, le district, les enseignants et les élèves, afin de préparer le terrain pour l'introduction d'une intervention de dépistage des abus sexuels sur les enfants dans les établissements scolaires des camps de Mahama, Mugombwa et Kigeme. Cette initiative, également développée et évaluée initialement au Kenya par Population Council et le Kenyatta National Hospital, est en attente en raison de la COVID-19.

\section{Partenaire(s) :}

Africa Humanitarian Action

Alight

Plan International

Population Council

Save the Children International

Pour de plus amples informations, contacter :

Zahra Migrani : mirghani@unhcr.org

\section{Étude de cas au Kenya : Gestion de la chaîne des preuves médico-légales}

$\square$ ans le cadre d'une réponse multisectorielle à la violence sexuelle et fondée sur le genre, l'accès à la justice/aux services juridiques est offert aux réfugiés survivants en fonction de leurs besoins et de leurs choix. Cependant, les poursuites sont souvent entravées par le manque de preuves médico-légales dans les affaires de viol et d'agression physique. Par conséquent, certaines affaires finissent par être rejetées par les tribunaux. Les services de police des districts de Kibondo et de Kasulu disposent d'unités médico-légales et sont prêts à apporter leur soutien aux camps de réfugiés. II existe cependant des lacunes dans les camps, notamment le manque de kits de soins post-viol dans tous les établissements de santé des camps, la nécessité de normaliser les formulaires des kits, la documentation inadéquate des formulaires de rapport d'examen médical de la police («PF3 ») et la mauvaise collecte des échantillons de preuves médico-légales.

Dans les commissariats de police, il y a un manque d'enquêteurs de police scientifique dans tous les camps, et un manque de fonds pour le traitement des preuves médico-légales de la scène au médecin légiste. En général, la coordination entre les acteurs de la police, de la protection et de la santé est faible, associée à un manque d'équipement pour la collecte et l'analyse des échantillons médico-légaux dans les centres de soutien aux survivants de violence sexuelle et fondée sur le genre.

En réponse à ces réalités, l'intervention actuelle vise à renforcer la gestion de la chaîne de collecte des preuves médicolégales en renforçant la capacité des prestataires de soins de santé et de police à collecter, documenter et utiliser les preuves médico-légales après un viol. L'intervention a été développée à l'origine par LVCT Health, Kenya, et comporte 


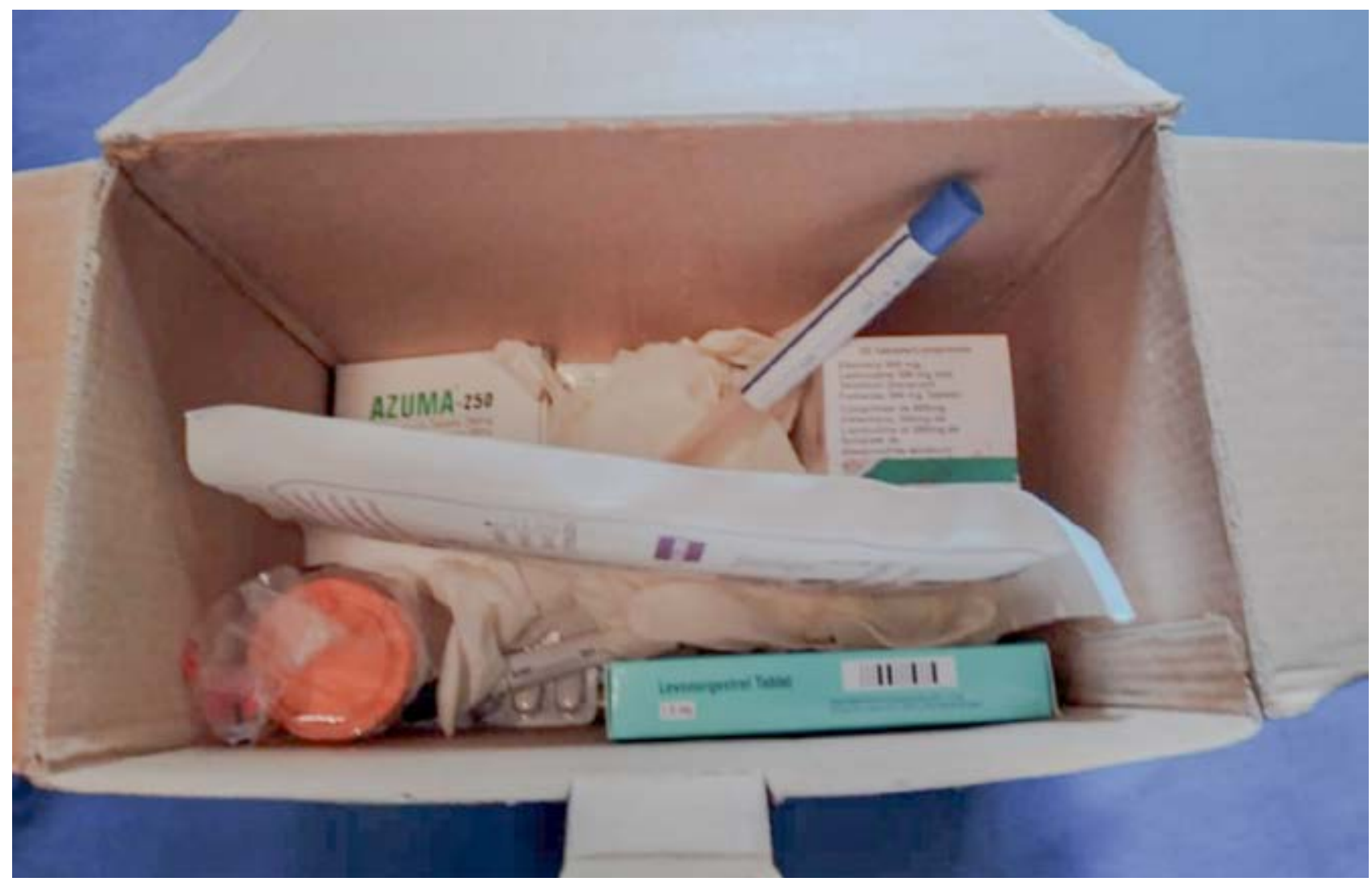

deux composantes principales : I'assemblage d'un kit de soin post-viol, en utilisant le matériel déjà disponible dans les établissements de santé (bien que dans des endroits différents dans ces contextes) ; et une formation multisectorielle pour les prestataires de santé utilisant le kit, de police et de droit/justice sur les formulaires nationaux de documentation du kit de soin post-viol qui sont censés être remplis en collaboration par les établissements de santé et les postes de police lorsqu'ils doivent traiter des cas de viol. Cette documentation doit être remplie avec précision pour faciliter la poursuite des affaires de viol devant les tribunaux. Comme les formulaires du dossier (formulaires de soins post-viol, formulaires d'examen médical de la police) contiennent souvent des sections à remplir par les prestataires de soins de santé et la police, il est important que tous maîtrisent les formulaires, sachent comment les remplir correctement, jouent un rôle de liaison entre les survivants et la police/l'établissement de santé (en fonction du lieu où le survivant s'est présenté en premier) et travaillent en collaboration pour garantir que les formulaires sont remplis en vue des procédures judiciaires.

Dans le contexte des réfugiés tanzaniens, cette intervention est mise en œuvre de la manière suivante :

- Des kits soin post-viol assemblés localement ont été mis en place par tous les établissements de santé.

- Le formulaire de prise en charge post-viol a été normalisé pour être utilisé dans tous les camps

- Une formation sur la collecte de preuves médico-légales et sur la manière de remplir le formulaire PF3 avec précision a été dispensée aux prestataires de santé de la prise en charge post-viol.

- La police a été chargée d'inclure un enquêteur de la scientifique dans l'examen d'un mémorandum d'accord entre le HCR et la police; d'allouer des fonds pour le traitement des preuves scientifiques en tous lieux; et d'acheter les articles du kit de soin post-viol qui n'étaient pas déjà disponibles dans les établissements de santé.

- L'unité de protection a été chargée de veiller à ce que les acteurs concernés assistent aux réunions mensuelles de coordination du groupe de travail sur la violence sexuelle et fondée sur le genre au niveau des camps, de contribuer à l'acquisition des équipements nécessaires et d'organiser des séances de sensibilisation aux bonnes pratiques en matière de preuves médico-légales.

- Six prestataires de soins de santé ont depuis été formés à la gestion de la chaîne médico-légale par le point focal des soins de santé. Les sujets de formation comprenaient la collecte et le stockage des preuves médico-légales, ainsi que les procédures d'assemblage des kits de soin post-viol.

- Un engagement coordonné entre le Ministère de l'intérieur, la police et le médecin légiste a été mené dans le laboratoire d'analyse régional de Mwanza. Un consensus s'est dégagé sur les prochaines étapes de l'intervention, notamment : des plans pour que le médecin légiste forme les travailleurs de la santé à la gestion de la chaîne des preuves médico-légales, et des plans pour que le gouvernement inspecte les établissements de santé afin de vérifier leur état de préparation à la gestion de la chaîne des preuves médico-légales (outils de collecte, stockage, etc.). 


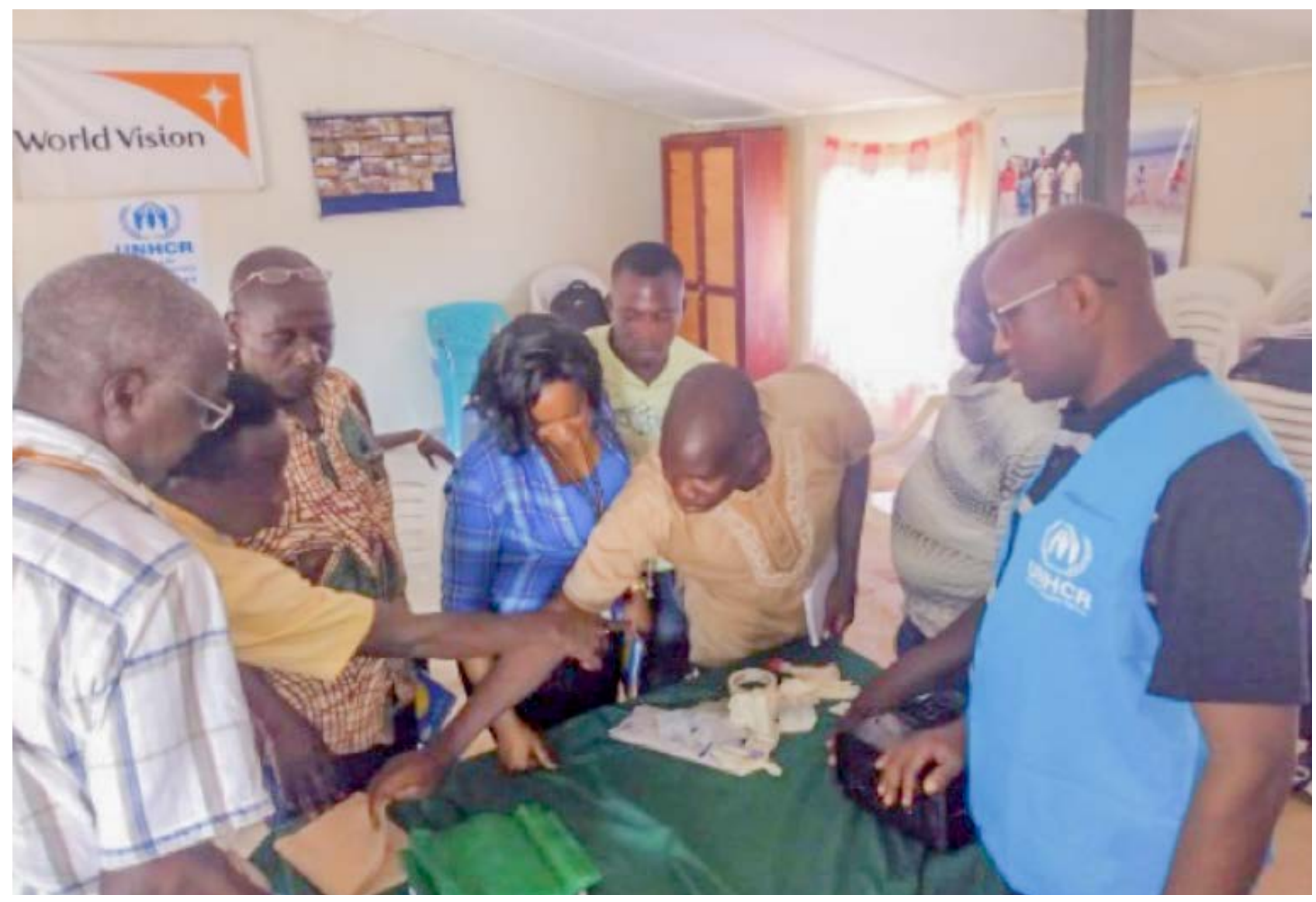

Depuis le début de l'intervention, on a constaté une amélioration dans le remplissage des formulaires PF3 par la police, bien que des sessions de recyclage périodiques soient recommandées en raison des affectations du personnel de police. Les kits de soin post-viol sont maintenant disponibles dans tous les établissements de santé des camps participants, avec des rapports périodiques et le soutien du HCR et des points focaux santé. L'amélioration de la coordination par l'unité de protection contre la violence sexuelle et fondée sur le genre a permis d'améliorer les mécanismes et les voies de référence. Des améliorations ont également été observées dans le temps de réponse aux survivants à divers points de prestation de services. Les réunions mensuelles organisées par le HCR avec les principaux acteurs chargés de faire le bilan de l'intervention (Comité international de secours, partenaires de santé, agents publics de protection, police) ont permis de trouver des solutions à certains des problèmes.

\section{Partenaire(s) :}

Comité international de secours

LVCT Health

Medical Teams International

Médecins Sans Frontières

Ministère de l'intérieur

La police

Partenaire d'exécution de la lutte contre la violence sexuelle et fondée sur le genre

La Croix-Rouge tanzanienne

Pour de plus amples informations, contacter:

Benon Orach : orachb@unhcr.org

Rehema Katyega : katyega@unhcr.org

DrYoel Mashimba :ymashimba@medicalteams.org

Miata Tubee Johnson : johnsonm@unhcr.org

Michael Gaitho : Michael.Gaitho@lvcthealth.org 


\section{Pratique prometteuse en Ouganda : L'« Alliance de villages de tolérance zéro »}

'«Alliance de villages de tolérance zéro » est une intervention communautaire de prévention de la violence sexuelle et fondée sur le genre qui a été développée pour les milieux ruraux sud-africains par le programme d'autonomisation des victimes de Thohoyandou, sous la direction de Fiona Nicholson, et introduite pour la première fois dans les milieux de réfugiés en Ouganda. Le camp de Rwamwanja est le pionnier de l'intervention sur un site de réfugiés, basé sur des taux élevés de violence sexuelle et fondée sur le genre.

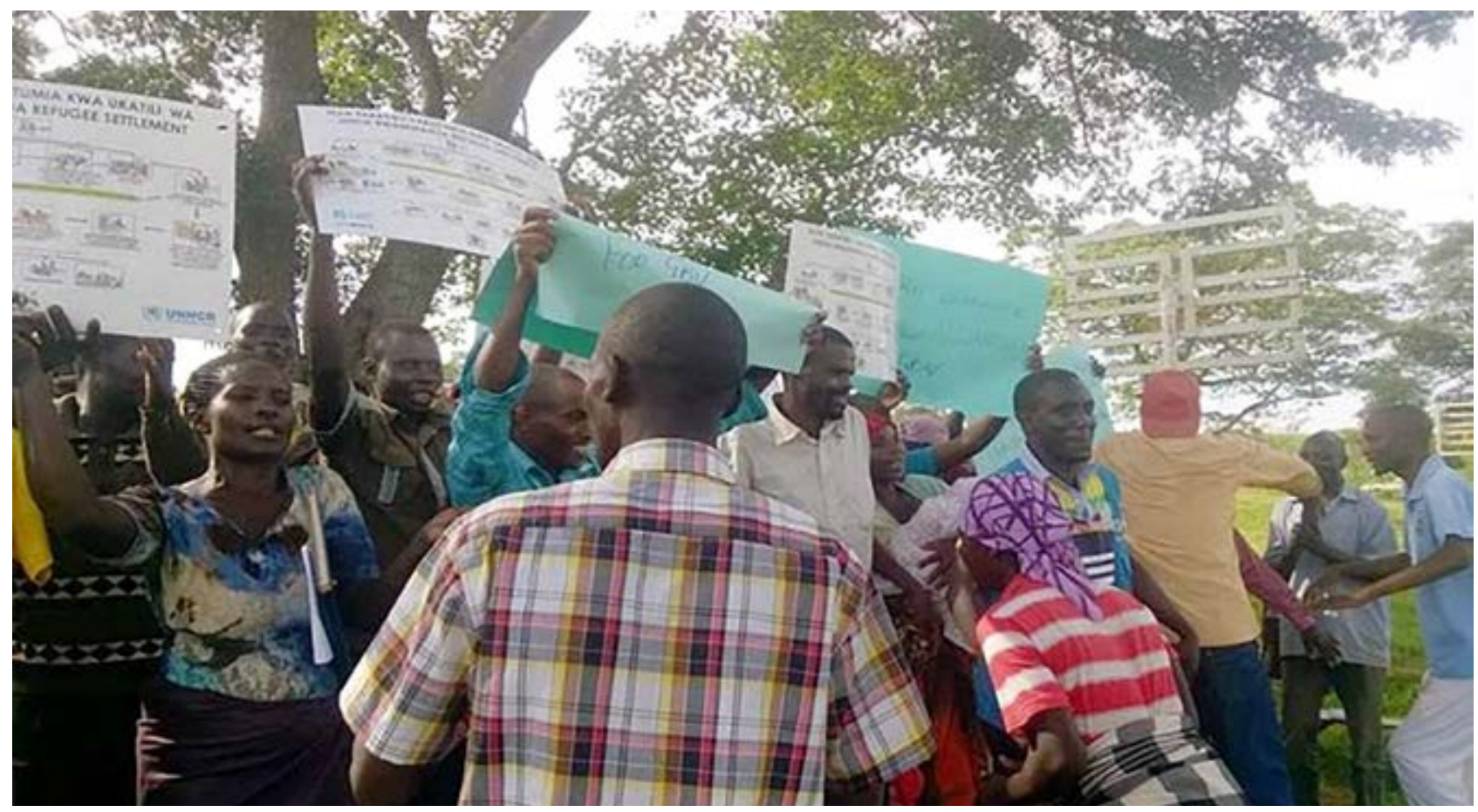

L'intervention comporte les éléments suivants :

Cartographie et dialogues communautaires : pour identifier toutes les structures et agences qui peuvent être ciblées pour la participation, y compris la fourniture de services de lutte contre la violence sexuelle et fondée sur le genre. Les dialogues communautaires sont utilisés pour fournir des informations sur l'intervention et promouvoir l'appropriation par la communauté.

Création d'un forum des parties prenantes : Un forum des parties prenantes est créé et ses membres sont représentatifs des secteurs de la communauté (c'est-à-dire les dirigeants communautaires, les églises, l'éducation, les entreprises). Le forum tient des réunions mensuelles pour évaluer la mise en œuvre de l'intervention et pour s'assurer que les membres de la communauté participent aux ateliers de formation dans le cadre de l'intervention.

Signature d'un mémorandum d'accord : Les membres du forum signent un mémorandum d'accord pour aider à formaliser et à guider le partenariat entre le forum et le partenaire d'exécution. Le mémorandum d'accord dresse une liste de critères que le forum aidera la communauté à remplir afin d'être officiellement intégrée dans une alliance de villages qui sont qualifiés de «zones de tolérance zéro » en matière de violence sexuelle et fondée sur le genre.

Formation : Les principaux groupes communautaires sont sensibilisés aux questions de la violence sexuelle et fondée sur le genre par le biais de sessions de formation. Des brochures résumant les formations sont fournies, et les stagiaires sont encouragés à former indépendamment deux autres membres de la communauté après avoir suivi leur propre formation individuelle.

Cérémonie d'engagement : Une fois que les attentes définies dans le mémorandum d'accord ont été satisfaites par le village, celui-ci reçoit le parrainage pour une cérémonie publique, au cours de laquelle les hommes de la communauté sont invités à s'engager publiquement à prendre une part active dans l'éradication de la violence sexuelle et fondée sur le genre dans leur village, et des récompenses sont décernées à divers membres de la communauté pour avoir pris position contre ce phénomène, ou pour avoir partagé leurs témoignages. 
Identification de l'alliance : Le point d'orgue de la cérémonie d'engagement est le dévoilement d'un panneau d'affichage communautaire, indiquant le nom du village et déclarant son statut de « tolérance zéro » et son intégration dans une « alliance » de villages de tolérance zéro. Une petite allocation a également été mise à la disposition des villages afin qu'ils puissent développer un sentiment d'appartenance à la communauté après la cérémonie.

Les résultats de l'évaluation de cette intervention dans les milieux de réfugiés ougandais ont démontré une réduction statistiquement importante des cas de violence physique exercée par le partenaire intime (VPI) (pour les hommes et les femmes), de la VPI sexuelle (pour les hommes), de la violence physique exercée par un non-partenaire (pour les hommes et les femmes) et de la violence sexuelle exercée par un non-partenaire (pour les femmes).

À ce jour, l'intervention de l'Alliance de villages de tolérance zéro a été mise en œuvre dans une quinzaine de villages de réfugiés en Ouganda. Des préparatifs de base (y compris la formation des partenaires d'exécution, la sensibilisation des dirigeants communautaires et/ou la création de forums des parties prenantes, etc.) ont également été effectués pour introduire l'intervention dans les milieux de réfugiés à Djibouti, en Éthiopie et en Zambie.

\section{Partenaire(s) :}

Fédération luthérienne mondiale

Cabinet du Premier ministre

Pour de plus amples informations, contacter :

Mildred Ouma : ouma@unhcr.org

Monicah Rwotmon : monicah.rwotmon@lutheranworld.org

Fiona Nicholson : nicholsonfiona27@gmail.com

\section{CÉtude de cas au Soudan du Sud : Approches communautaires en matière de protection (prévention de la violence sexuelle et fondée sur le genre, atténuation des risques, protection des enfants et engagement des jeunes)}

es réfugiés de l'État du Haut-Nil résident dans quatre camps du comté de Maban, et dépendent fortement des agences humanitaires pour la fourniture de services de base et de survie. Bien que des progrès significatifs aient été réalisés, des préoccupations liées aux incidents de violence sexuelle et fondée sur le genre, tels que les mariages forcés, les viols, les mutilations génitales féminines/excision et le déni de possibilités aux femmes et aux filles continuent d'être signalées. En outre, la présence d'éléments armés a compromis le caractère civil et humanitaire de l'asile et a contribué à la survenance de certains incidents de violence sexuelle et fondée sur le genre.

Une intervention à plusieurs facettes a donc été mise en place avec les objectifs suivants : assurer la sécurité des victimes de violence sexuelle et fondée sur le genre dans les camps de réfugiés de Maban (y compris, sans toutefois s'y limiter, en fournissant des abris sûrs au sein de la communauté) ; améliorer l'interaction sociale, établir ou renforcer les réseaux sociaux et instaurer la confiance dans la défense des droits et le partage des informations; et renforcer la capacité des structures communautaires à identifier, orienter et répondre en temps utile aux besoins qui se font jour dans la communauté.

Afin d'engager efficacement les structures communautaires dans la réalisation de ces objectifs, le HCR, en collaboration avec les partenaires en matière de violence sexuelle et fondée sur le genre et de protection de l'enfance et de la jeunesse, a continué à renforcer les capacités des défenseurs de la lutte contre la violence sexuelle et fondée sur le genre, SASA ! Des activistes, des responsables de réfugiés, des représentants des femmes et des jeunes, des activistes communautaires et des représentants de centres d'hébergement communautaires sécurisés afin de renforcer leur contribution à la prévention, à l'atténuation et à la réponse aux problèmes de violence sexuelle et fondée sur le genre en coordination avec le HCR et ses partenaires. Les interventions spécifiques comprennent:

- La création de centres d'hébergement communautaires sécurisés - une initiative communautaire - pour garantir que les survivants ne soient pas exposés à des risques.

- Les activités du Centre des femmes (sessions d'écoute pour les femmes et les filles), qui offrent une plate-forme sûre pour discuter des questions qui les concernent et pour partager des recommandations allant dans le sens du traitement de ces questions. 
- Un engagement de toute la société dans la réponse et la prévention de la violence sexuelle et fondée sur le genre, avec une inclusion particulière des jeunes et des enfants dans les espaces où ils vivent, jouent, travaillent et apprennent.

- Un programme solide de lutte contre la violence sexuelle et fondée sur le genre, impliquant la méthodologie SASA!, une approche communautaire de la société dans son ensemble, dans laquelle les militants SASA !, les observateurs de la protection, les défenseurs de la lutte contre la violence sexuelle et fondée sur le genre, les dirigeants communautaires, les représentants d'associations et les chefs religieux sont tous engagés dans la sensibilisation et la diffusion d'informations sur ce phénomène au niveau du village.

- L'engagement délibéré des garçons et des hommes dans le programme de prévention de la violence sexuelle et fondée sur le genre en tant que catalyseurs d'un changement positif de comportement.

- Gestion des cas par des points focaux partenaires assurant une réponse multisectorielle.

- Fourniture de tentes et d'articles non alimentaires.

\section{Partenaire(s) :}

Commission for Refugee Affairs

Autorités du comté

Conseil danois pour les réfugiés

Ministry of Gender, Child, and Social Welfare

Relief International

Save the Children International

\section{Pour de plus amples informations, contacter :}

Domaine de responsabilité SO Bunj 
